\title{
ORBITAL AND COLLISIONAL EVOLUTION OF THE IRREGULAR SATELLITES
}

\author{
David Nesvorný, ${ }^{1}$ Jose L. A. Alvarellos, ${ }^{2}$ Luke Dones, ${ }^{1}$ And Harold F. Levison ${ }^{1}$ \\ Received 2002 December 10; accepted 2003 March 18
}

\begin{abstract}
The irregular moons of the Jovian planets are a puzzling part of the solar system inventory. Unlike regular satellites, the irregular moons revolve around planets at large distances in tilted and eccentric orbits. Their origin, which is intimately linked with the origin of the planets themselves, is yet to be explained. Here we report a study of the orbital and collisional evolution of the irregular satellites from times after their formation to the present epoch. The purpose of this study is to find out the features of the observed irregular moons that can be attributed to this evolution and separate them from signatures of the formation process. We numerically integrated $\approx 60,000$ test satellite orbits to map orbital locations that are stable on long time intervals. We found that the orbits highly inclined to the ecliptic are unstable due to the effect of the Kozai resonance, which radially stretches them so that satellites either escape from the Hill sphere, collide with massive inner moons, or impact the parent planet. We also found that prograde satellite orbits with large semimajor axes are unstable due to the effect of the evection resonance, which locks the orbit's apocenter to the apparent motion of the Sun around the parent planet. In such a resonance, the effect of solar tides on a resonant moon accumulates at each apocenter passage of the moon, which causes a radially outward drift of its orbital apocenter; once close to the Hill sphere, the moon escapes. By contrast, retrograde moons with large orbital semimajor axes are long-lived. We have developed an analytic model of the distant satellite orbits and used it to explain the results of our numerical experiments. In particular, we analytically studied the effect of the Kozai resonance. We numerically integrated the orbits of the 50 irregular moons (known by 2002 August 16) for $10^{8} \mathrm{yr}$. All orbits were stable on this time interval and did not show any macroscopic variations that would indicate instabilities operating on longer time spans. The average orbits calculated from this experiment were then used to probe the collisional evolution of the irregular satellite systems. We found that (1) the large irregular moons must have collisionally eliminated many small irregular moons, thus shaping their population to the currently observed structures; (2) some dynamical families of satellites could have been formed by catastrophic collisions among the irregular moons; and (3) Phoebe's surface must have been heavily cratered by impacts from an extinct population of Saturnian irregular moons, much larger than the present one. We therefore suggest that the Cassini imaging of Phoebe in 2004 can be used to determine the primordial population of small irregular moons of Saturn. In such a case, we will also better understand the overall efficiency of the formation process of the irregular satellites and the physical conditions that existed during planetary formation. We discovered two dynamical families of tightly clustered orbits within the Jovian retrograde group. We believe that these two clusters may be the remnants of two collisionally disrupted bodies. We found that the entire Jovian retrograde group and the Saturnian inclination groups were not produced by single breakups, because the ejection velocities derived from the orbital structures of these groups greatly exceed values calculated by modern numerical models of collisional breakups. Taken together, the evidence presented here suggests that many properties of the irregular moons previously assigned to their formation process may have resulted from their later dynamical and collisional evolution. Finally, we have found that several irregular moons, namely, Pasiphae, Sinope, S/2001 J10, S/2000 S5, S/2000 S6, and S/2000 S3, have orbits characterized by secular resonances. The orbits of some of these moons apparently evolved by some slow dissipative process in the past and became captured in tiny resonant volumes.
\end{abstract}

Key words: astrometry — celestial mechanics — planets and satellites: formation — planets and satellites: general

On-line material: color figures

\section{INTRODUCTION}

This paper deals with irregular satellites of the Jovian planets. We informally define the irregular satellites as those moons that are far enough from their parent planet that the precession of their orbital plane is primarily controlled by the Sun. This implies that a satellite with a semimajor axis $a$ greater than $a_{\text {crit }} \approx\left(2 \mu J_{2} R^{2} a_{p}^{3}\right)^{1 / 5}$ is an irregular satellite (Burns 1986). In this expression, $\mu$ is the ratio of the planet's

${ }^{1}$ Southwest Research Institute, Suite 400, 1050 Walnut Street, Boulder, CO 80302 .

${ }^{2}$ Space Systems/Loral MS L-27, 3825 Fabian Way, Palo Alto, CA 94303. mass to the Sun's, $J_{2}$ is the planet's second zonal harmonic coefficient, $R$ is the planet's equatorial radius, and $a_{p}$ is the planet's semimajor axis. According to this definition, we currently ${ }^{3}$ know of 31 irregular satellites at Jupiter, 13 at Saturn, five at Uranus, and one at Neptune (Nereid). ${ }^{4}$ Note that our definition excludes Neptune's large moon, Triton.

\footnotetext{
${ }^{3}$ By 2002 August 16.

${ }^{4}$ By 2003 March 9, these numbers increased: we know of 12 new irregular satellites of Jupiter (S/2003 J1-J12; Sheppard 2003a, 2003b, 2003c); S/2001 U1 (Holman, Kavelaars, \& Milisavljevic 2002) is the sixth known irregular moon of Uranus; Neptune has gained three new irregular moons (S/2002 N1-N3; Holman et al. 2003). The orbits of these new moons are yet to be precisely determined.
} 
EVOLUTION OF THE IRREGULAR SATELLITES

TABLE 1

Orbits and Sizes of the Irregular Moons of Jupiter as of 2002 August 16

\begin{tabular}{|c|c|c|c|c|c|c|c|c|}
\hline ID & Satellite & $\begin{array}{c}i \\
(\operatorname{deg})\end{array}$ & $e$ & $\begin{array}{c}a \\
(\mathrm{AU})\end{array}$ & $\begin{array}{c}a \\
\left(R_{j}^{\mathrm{H}}\right)\end{array}$ & $\begin{array}{l}T_{\text {orb }} \\
(\mathrm{yr})\end{array}$ & $\begin{array}{c}H \\
(\mathrm{mag})\end{array}$ & $\begin{array}{c}D \\
(\mathrm{~km})\end{array}$ \\
\hline \multicolumn{9}{|c|}{ Jupiter $\left(R_{5}^{\mathrm{H}}=0.355 \mathrm{AU}\right)$ : } \\
\hline $1 \ldots \ldots \ldots \ldots \ldots$ & $\mathrm{S} / 1975 \mathrm{~J} 1$ & 45.7 & 0.204 & 0.049 & 0.138 & 0.3554 & 14.5 & 8 \\
\hline $2 \ldots \ldots \ldots \ldots \ldots$ & J13 (Leda) & 26.7 & 0.147 & 0.074 & 0.208 & 0.6530 & 13.5 & 15 \\
\hline $3 \ldots \ldots \ldots \ldots \ldots$ & J6 (Himalia) & 30.2 & 0.166 & 0.076 & 0.214 & 0.6787 & 8.1 & $120 \times 150$ \\
\hline $4 \ldots \ldots \ldots$ & J7 (Elara) & 28.9 & 0.221 & 0.078 & 0.220 & 0.7113 & 10.0 & 75 \\
\hline $5 \ldots \ldots \ldots \ldots \ldots$ & J10 (Lysithea) & 27.8 & 0.104 & 0.079 & 0.223 & 0.7124 & 11.7 & 35 \\
\hline $6 \ldots \ldots \ldots \ldots \ldots$ & $\mathrm{S} / 2000 \mathrm{~J} 11$ & 28.2 & 0.208 & 0.083 & 0.234 & 0.7784 & 16.0 & 4 \\
\hline $7 \ldots \ldots \ldots \ldots \ldots$ & $\mathrm{S} / 2000 \mathrm{~J} 3$ & 148.5 & 0.233 & 0.133 & 0.375 & 1.57 & 15.2 & 6 \\
\hline $8 \ldots \ldots \ldots \ldots \ldots$ & $\mathrm{S} / 2000 \mathrm{~J} 7$ & 145.7 & 0.127 & 0.137 & 0.386 & 1.63 & 14.6 & 7 \\
\hline $9 \ldots \ldots \ldots$ & $\mathrm{S} / 2000 \mathrm{~J} 5$ & 149.2 & 0.239 & 0.136 & 0.383 & 1.63 & 15.5 & 5 \\
\hline $10 \ldots \ldots \ldots \ldots$ & J12 (Ananke) & 147.0 & 0.252 & 0.140 & 0.394 & 1.70 & 12.2 & 30 \\
\hline $11 \ldots \ldots \ldots \ldots$ & $\mathrm{S} / 2000 \mathrm{~J} 10$ & 165.5 & 0.198 & 0.149 & 0.420 & 1.86 & 15.6 & 5 \\
\hline $12 \ldots \ldots \ldots \ldots$ & J11 (Carme) & 166.3 & 0.263 & 0.148 & 0.417 & 1.84 & 11.3 & 40 \\
\hline $13 \ldots \ldots \ldots \ldots$ & $\mathrm{S} / 2000 \mathrm{~J} 9$ & 163.8 & 0.189 & 0.150 & 0.422 & 1.88 & 15.2 & 6 \\
\hline $14 \ldots \ldots \ldots \ldots$ & $\mathrm{S} / 2000 \mathrm{~J} 4$ & 161.3 & 0.323 & 0.152 & 0.428 & 1.92 & 15.9 & 4 \\
\hline $15 \ldots \ldots \ldots \ldots$ & $\mathrm{S} / 2000 \mathrm{~J} 6$ & 164.8 & 0.315 & 0.155 & 0.437 & 1.97 & 15.8 & 4 \\
\hline $16 \ldots \ldots \ldots \ldots$ & J8 (Pasiphae) & 140.0 & 0.380 & 0.156 & 0.439 & 2.00 & 10.3 & 50 \\
\hline $17 \ldots \ldots \ldots \ldots$ & J9 (Sinope) & 158.0 & 0.370 & 0.158 & 0.445 & 2.04 & 11.6 & 35 \\
\hline $18 \ldots \ldots \ldots \ldots$ & $\mathrm{S} / 2000 \mathrm{~J} 2$ & 166.3 & 0.267 & 0.161 & 0.454 & 2.09 & 15.1 & 6 \\
\hline $19 \ldots \ldots \ldots \ldots$ & $\mathrm{S} / 2000 \mathrm{~J} 8$ & 150.3 & 0.575 & 0.163 & 0.459 & 2.12 & 14.8 & 6 \\
\hline $20 \ldots \ldots \ldots \ldots$ & $\mathrm{S} / 1999 \mathrm{~J} 1$ & 142.3 & 0.107 & 0.165 & 0.466 & 2.17 & 14.2 & 10 \\
\hline $21 \ldots \ldots \ldots \ldots$ & $\mathrm{S} / 2001 \mathrm{~J} 10$ & 147.0 & 0.156 & 0.127 & 0.358 & 1.46 & 16.5 & 2 \\
\hline $22 \ldots \ldots \ldots \ldots$ & $\mathrm{S} / 2001 \mathrm{~J} 8$ & 165.3 & 0.475 & 0.138 & 0.389 & 1.67 & 16.4 & 2 \\
\hline $23 \ldots \ldots \ldots \ldots$ & $\mathrm{S} / 2001 \mathrm{~J} 9$ & 141.9 & 0.272 & 0.139 & 0.392 & 1.69 & 16.5 & 2 \\
\hline $24 \ldots \ldots \ldots \ldots$ & $\mathrm{S} / 2001 \mathrm{~J} 2$ & 149.0 & 0.295 & 0.139 & 0.392 & 1.68 & 15.7 & 4 \\
\hline $25 \ldots \ldots \ldots \ldots$ & $\mathrm{S} / 2001 \mathrm{~J} 7$ & 145.9 & 0.176 & 0.140 & 0.394 & 1.70 & 16.2 & 3 \\
\hline $26 \ldots \ldots \ldots \ldots$ & $\mathrm{S} / 2001 \mathrm{~J} 3$ & 150.3 & 0.251 & 0.142 & 0.400 & 1.72 & 15.5 & 4 \\
\hline $27 \ldots \ldots \ldots \ldots$ & $\mathrm{S} / 2001 \mathrm{~J} 6$ & 165.4 & 0.288 & 0.153 & 0.431 & 1.96 & 16.6 & 2 \\
\hline $28 \ldots \ldots \ldots \ldots$ & $\mathrm{S} / 2001 \mathrm{~J} 4$ & 150.1 & 0.345 & 0.156 & 0.439 & 1.95 & 16.1 & 3 \\
\hline $29 \ldots \ldots \ldots \ldots$ & $\mathrm{S} / 2001 \mathrm{~J} 11$ & 165.7 & 0.291 & 0.156 & 0.439 & 2.01 & 16.1 & 3 \\
\hline $30 \ldots \ldots \ldots \ldots$ & $\mathrm{S} / 2001 \mathrm{~J} 5$ & 155.0 & 0.454 & 0.157 & 0.442 & 2.00 & 16.4 & 2 \\
\hline $31 \ldots \ldots \ldots \ldots$ & $\mathrm{S} / 2001 \mathrm{~J} 1$ & 151.9 & 0.415 & 0.159 & 0.448 & 2.06 & 15.4 & 4 \\
\hline
\end{tabular}

Notes.-The columns are identification number (ID), name of the satellite, inclination $(i$, measured with respect to the ecliptic), eccentricity $(e)$, semimajor axis $(a)$ in $\mathrm{AU}$ and in Hill radii $\left(R_{5}^{\mathrm{H}} \approx 0.355 \mathrm{AU}\right)$, orbital period ( $\left.T_{\text {orb }}\right)$, absolute magnitude $(H)$, and estimated diameter $(D)$. The albedo $A$ used to convert $H$ into $D$ is 0.04 (Rettig et al. 2001), except for Himalia, which has an irregular shape and $A=0.05$ (Porco et al. 2003). The satellites were ordered by increasing semimajor axis. The 11 irregular moons that were announced on 2002 May 16 (Sheppard et al. 2002) were listed separately (numbered 21-31; Sheppard et al. 2002). Source: http://ssd.jpl.nasa.gov/sat_elem.html and http://ssd.jpl.nasa.gov/sat_props.html.

Tables 1 and 2 list the physical and orbital properties of the irregular satellites. ${ }^{5}$ Figure 1 shows their current orbits. In the following, we briefly summarize what we know about the irregular satellites.

Jupiter.-Jupiter's irregulars come in two groups: six prograde and 25 retrograde moons. ${ }^{6}$ Five of the prograde moons move on similar orbits with semimajor axes $a \sim(0.21-0.23) R^{\mathrm{H}}$, eccentricities $e \sim 0.1-0.2$, and inclinations to the ecliptic (or invariable plane) $i \sim 30^{\circ}$. Here $R^{\mathrm{H}}=a_{p}(\mu / 3)^{1 / 3}$ is the Hill radius of the planet $(\approx 0.355 \mathrm{AU}$ for Jupiter; Table 1). The sixth prograde moon, S/1975 J1 (Themisto), has a smaller orbital distance from Jupiter $\left(a \sim 0.14 R^{\mathrm{H}}\right)$. The orbits of the retrograde moons have larger semimajor axes $\left[a \sim(0.36-0.47) R^{\mathrm{H}}\right]$, are more eccentric than the orbits of the prograde moons, and have $i \sim 140^{\circ}-167^{\circ}$.

\footnotetext{
${ }^{5}$ See http://ssd.jpl.nasa.gov/sat_elem.html and http://ssd.jpl.nasa.gov/sat_props.html for up-to-date lists.

${ }^{6}$ Twelve new moons, S/2003 J1-J12, all seem to be retrograde.
}

Assuming an albedo of 0.04 , the diameters $(D)$ of the six prograde moons range from 4 to $75 \mathrm{~km}$ (Rettig, Walsh, \& Consolmagno 2001), except for large Himalia, which is $\sim 150 \times 120 \mathrm{~km}$ across, with an albedo $A=0.05$ (Porco et al. 2003). By contrast, the retrograde moons are smaller (2$60 \mathrm{~km}$ in diameter; see Sheppard \& Jewitt 2002). The prograde moons spectrally resemble each other. Their visual colors are gray like the colors of the C-type asteroids (Sykes et al. 2000, Rettig et al. 2001). It is thus conceivable that the prograde moons are fragments of a captured C-type asteroid. The retrograde moons exhibit much greater color diversity than the prograde satellites. Carme, Pasiphae, and Sinope are redder, possibly resembling D-type Trojan asteroids. The near-infrared colors of Sinope are significantly different from those of Carme and Pasiphae; Ananke is unusually faint in the near-infrared (Sykes et al. 2000). Moreover, Carme shows a peculiar upturn in the ultraviolet part of its spectrum, which distinguishes it from other satellites (Tholen \& Zellner 1984). Taken together, the greater diversity among retrograde satellites suggests a more 
TABLE 2

Orbits and Sizes of the Irregular Moons of Saturn, Uranus, and Neptune as of 2002 August 16

\begin{tabular}{|c|c|c|c|c|c|c|c|c|}
\hline ID & Satellite & $\begin{array}{c}i \\
(\mathrm{deg})\end{array}$ & $e$ & $\begin{array}{c}a \\
(\mathrm{AU})\end{array}$ & $\begin{array}{c}a \\
\left(R_{j}^{\mathrm{H}}\right)\end{array}$ & $\begin{array}{l}T_{\text {orb }} \\
\text { (yr) }\end{array}$ & $\begin{array}{c}H \\
(\mathrm{mag})\end{array}$ & $\begin{array}{c}D \\
(\mathrm{~km})\end{array}$ \\
\hline \multicolumn{9}{|c|}{ Saturn $\left(R_{6}^{\mathrm{H}}=0.438 \mathrm{AU}\right)$ : } \\
\hline $1 \ldots \ldots \ldots \ldots \ldots$ & $\mathrm{S} / 2000 \mathrm{~S} 5$ & 48.5 & 0.164 & 0.076 & 0.174 & 1.24 & 12.3 & 17 \\
\hline $2 \ldots \ldots \ldots \ldots \ldots$ & $\mathrm{S} / 2000 \mathrm{~S} 6$ & 49.2 & 0.365 & 0.076 & 0.174 & 1.24 & 12.8 & 14 \\
\hline $3 \ldots \ldots \ldots \ldots \ldots$ & Phoebe & 173.2 & 0.175 & 0.086 & 0.196 & 1.49 & 6.9 & 240 \\
\hline $4 \ldots \ldots \ldots \ldots \ldots$ & $\mathrm{S} / 2000 \mathrm{~S} 2$ & 47.1 & 0.462 & 0.100 & 0.228 & 1.87 & 11.5 & 25 \\
\hline $5 \ldots \ldots \ldots \ldots \ldots$ & $\mathrm{S} / 2000 \mathrm{~S} 8$ & 148.6 & 0.210 & 0.104 & 0.237 & 1.98 & 13.8 & 8 \\
\hline $6 \ldots \ldots \ldots \ldots \ldots$ & $\mathrm{S} / 2000 \mathrm{~S} 3$ & 48.5 & 0.339 & 0.114 & 0.260 & 2.27 & 10.2 & 45 \\
\hline $7 \ldots \ldots \ldots \ldots \ldots$ & $\mathrm{S} / 2000 \mathrm{~S} 10$ & 34.2 & 0.616 & 0.116 & 0.265 & 2.34 & 13.3 & 10 \\
\hline $8 \ldots \ldots \ldots \ldots \ldots$ & $\mathrm{S} / 2000 \mathrm{~S} 11$ & 34.9 & 0.381 & 0.119 & 0.272 & 2.43 & 10.9 & 30 \\
\hline $9 \ldots \ldots \ldots \ldots \ldots$ & $\mathrm{S} / 2000 \mathrm{~S} 4$ & 34.9 & 0.637 & 0.120 & 0.274 & 2.47 & 12.5 & 16 \\
\hline $10 \ldots \ldots \ldots \ldots$ & $\mathrm{S} / 2000 \mathrm{~S} 9$ & 169.5 & 0.265 & 0.123 & 0.280 & 2.55 & 14.1 & 7 \\
\hline $11 \ldots \ldots \ldots \ldots$ & $\mathrm{S} / 2000 \mathrm{~S} 12$ & 174.7 & 0.107 & 0.131 & 0.299 & 2.82 & 14.0 & 7 \\
\hline $12 \ldots \ldots \ldots \ldots$ & $\mathrm{S} / 2000 \mathrm{~S} 7$ & 174.9 & 0.532 & 0.137 & 0.313 & 3.01 & 14.0 & 7 \\
\hline $13 \ldots \ldots \ldots \ldots$ & $\mathrm{S} / 2000 \mathrm{~S} 1$ & 172.9 & 0.385 & 0.156 & 0.356 & 3.63 & 11.9 & 20 \\
\hline \multicolumn{9}{|c|}{ Uranus $\left(R_{7}^{\mathrm{H}}=0.469\right) \mathrm{AU}$ : } \\
\hline $1 \ldots \ldots \ldots \ldots$ & S/1997 U1 (Caliban) & 139.6 & 0.081 & 0.048 & 0.102 & 1.59 & 8.8 & 60 \\
\hline $2 \ldots \ldots \ldots \ldots \ldots$ & S/1999 U2 (Stephano) & 141.6 & 0.145 & 0.053 & 0.113 & 1.84 & 10.9 & 20 \\
\hline $3 \ldots \ldots \ldots \ldots \ldots$ & S/1997 U2 (Sycorax) & 152.9 & 0.504 & 0.081 & 0.173 & 3.53 & 7.1 & 120 \\
\hline $4 \ldots \ldots \ldots \ldots \ldots$ & S/1999 U3 (Prospero) & 146.3 & 0.324 & 0.110 & 0.235 & 5.50 & 10.1 & 30 \\
\hline $5 \ldots \ldots \ldots \ldots \ldots$ & S/1999 U1 (Setebos) & 147.9 & 0.522 & 0.119 & 0.253 & 6.25 & 10.0 & 30 \\
\hline \multicolumn{9}{|c|}{ Neptune $\left(R_{8}^{\mathrm{H}}=0.777 \mathrm{AU}\right)$} \\
\hline $1 \ldots \ldots \ldots \ldots$ & Nereid & 7.3 & 0.751 & 0.037 & 0.048 & 0.99 & 4.6 & 340 \\
\hline
\end{tabular}

Notes.-The columns are the same as in Table 1. The albedo $A$ used to convert $H$ into $D$ is $A=0.05$ for the Saturnian irregular moons (Gladman et al. 2001); and $A=0.07$ for the Uranian irregulars (Gladman et al. 1998, 1999). The size of Nereid was taken from Thomas, Veverka, \& Helfenstein 1991. The data come from Gladman et al. 1998, 1999, 2001, and Jacobson 2000, 2001. The satellites are ordered by increasing semimajor axis.

heterogeneous parent body or a capture and subsequent fragmentation of more than one parent body.

Kessler (1981) found that the prograde irregular Jovian satellites have a lifetime against collisions with each other of $\sim 1$ billion years, while the retrograde moons are unlikely to have collided in the age of the solar system. These collision rates were computed by Kessler for the four prograde and four retrograde moons known at that time. By contrast, a total population of $\sim 100$ Jovian irregulars with $D>1 \mathrm{~km}$ is inferred from modern observational surveys (Sheppard \& Jewitt 2002). The collisional evolution of the Jovian irregular moons may thus be more important than calculated by Kessler (1981) due to the large number of bodies moving on crossing orbits.

Saturn.-Saturn's 13 irregulars form three groups of moons with similar inclinations: the $34^{\circ}$ inclination group (three members), the $46^{\circ}$ group (four members), and the Phoebe group (six members). The two former groups were proposed by Gladman et al. (2001) to be remnants of larger satellites that were collisionally disrupted after capture. The semimajor axes of Saturn's irregular moons range between $0.17 R^{\mathrm{H}}$ and $0.36 R^{\mathrm{H}}$. The moons are $6-32 \mathrm{~km}$ across (assuming $6 \%$ albedo), except for the large retrograde moon Phoebe, which is $\approx 220 \mathrm{~km}$ in diameter. Phoebe has a neutral color (Simonelli et al. 1999); in addition, water ice was recently detected on Phoebe (Owen et al. 1999). Owen et al. argued that this implies that Phoebe originated in the outer solar system, not as an escaped C-type main-belt asteroid as proposed by Hartmann (1987). Phoebe will be imaged by the Cassini camera at sub-kilometer resolution as the spacecraft approaches Saturn in 2004. Gladman et al. (2001) suggested that the Phoebe group of moons was created by a cratering impact on Phoebe's surface. If so, the Cassini imaging should reveal a large crater on Phoebe that may have been formed by this event.

Uranus.-Uranus's five known irregular moons move on retrograde orbits (Gladman et al. 1998, 1999). ${ }^{7}$ Their diameters range between 20 and $120 \mathrm{~km}$, assuming a $7 \%$ albedo. The moons have a red color (Gladman et al. 1998). Their orbits are retrograde and have $a \sim(0.1-0.25) R^{\mathrm{H}}$. The large semimajor axis spread of the irregular moons of Uranus suggests that these bodies formed independently, rather than having a common origin in a few parent bodies. This situation contrasts with the irregular moon systems of Jupiter and Saturn, which are clustered at a few orbital locations. In the latter case, the observed moons may be the largest members of groups that consist of (yet to be discovered) sub- $10 \mathrm{~km}$ bodies.

Neptune.--Neptune's moon Nereid has a diameter of 340 $\mathrm{km}$ and a neutral or slightly blue color (Thomas, Veverka, $\&$ Helfenstein 1996). It has the highest eccentricity $(\sim 0.75)$ among all known irregular satellites. Nereid's orbit is prograde with an inclination of only $7^{\circ}$ to Neptune's orbital plane. Nereid's highly eccentric orbit was hypothesized to have originated from perturbations by captured Triton, whose migrating orbit could have also destabilized many other moons of Neptune (Goldreich et al. 1989). As with Phoebe, water ice was recently detected on Nereid (Brown et al. 1999). The spectrum and albedo of Nereid appear intermediate between those of the Uranian satellites Umbriel and Oberon, suggesting a surface composed of a combination of water ice frost and a dark and spectrally neutral material. According to Brown et al. (1999), the

\footnotetext{
${ }^{7}$ The newly discovered moon, $\mathrm{S} / 2001 \mathrm{U} 1$, is also retrograde.
} 

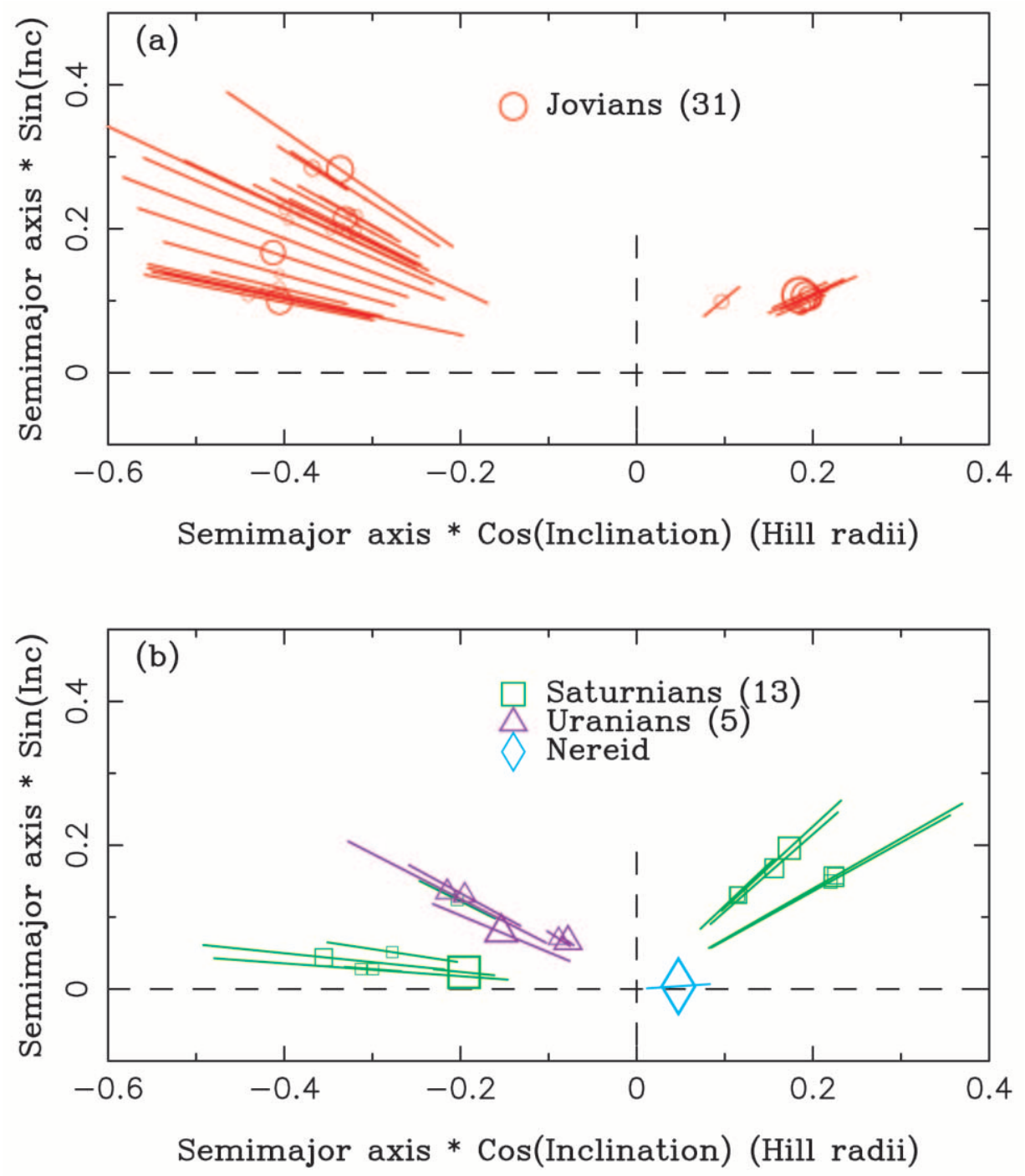

FIG. 1.-Orbits and sizes of 50 irregular moons as of 2002 August 16: (a) Jovian satellites (31 objects) and (b) Saturnians (13), Uranians (5), and Nereid. Jovian satellites form two large groups with prograde and retrograde orbits. Saturnians are distributed in three groups, with members of each group having similar inclinations. Uranian irregulars have retrograde orbits. The size of each symbol is proportional to the size of the object. The center of each symbol denotes the orbital elements of a satellite: the semimajor axes and inclinations are shown in polar coordinates, with the orbital inclination being the angle counted from the positive $X$-axis. The planetocentric distance of each moon varies in the range shown by the line segments extending radially from the coordinate center. The length of a segment is $2 a e$, where $a$ is the semimajor axis and $e$ is the eccentricity of the corresponding moon. Inclination is measured with respect to the ecliptic.

spectrum thus provides support for the hypothesis that Nereid is a satellite formed in a circumplanetary environment rather than a captured object.

Gladman et al. (2000) searched most of Neptune's Hill sphere for satellites and found that Neptune probably does not have other irregular satellites brighter than an $R$ magnitude of 23.5 , corresponding to a diameter of $70 \mathrm{~km}$ for an assumed 7\% albedo. Holman et al. (2001) searched the region within $\sim 36^{\prime}$ of Neptune to a magnitude limit of $R \sim 25.5$ and found two candidate satellites of Neptune. Finally, on 2003 January 13, three new irregular satellites were announced by the same observers (Holman et al. 2003). S/2002 N1, N2, and N3 have substantially larger orbits than Nereid: $a \sim 0.13-0.15$ AU (Nereid has $a \sim 0.037 \mathrm{AU}) . \mathrm{S} / 2002 \mathrm{~N} 1$ is retrograde and probably has the largest orbital tilt with respect to the ecliptic of all natural moons $\left(i \sim 120^{\circ}\right) . \mathrm{S} / 2002 \mathrm{~N} 2$ and $\mathrm{S} / 2002 \mathrm{~N} 3$ have prograde orbits and similarly large orbital inclinations
( $i \sim 57^{\circ}$ and $\sim 43^{\circ}$, respectively). Orbital eccentricities of these $\sim 20-50 \mathrm{~km}$ diameter moons are yet to be determined.

As a general characteristic of the orbital distribution of the irregular satellites, we note that no moon has an inclination in the range between $\sim 50^{\circ}$ and $\sim 140^{\circ}$ (except for the new moons of Neptune, S/2002 N1 and S/2002 N2; Fig. 1). This is probably an effect of the so-called Kozai resonance, which destabilizes moons with these inclinations either by decreasing their pericenter distance $q=a(1-e)$ within the reach of the massive inner moons, to $q<R$ (where $R$ is the planetary radius), or by driving the apocenter $Q=a(1+e)$ to distances where solar tides outweigh the planet's gravity (Carruba et al. 2002b). We also note that the closer the planet is to the Sun, the more distant are its irregular satellites when expressed in Hill radii. This may be one signature of the formation process of irregular satellites that can be gleaned from their present orbits, because long-term orbital instabilities are not expected to have such a selective effect. 
The standard model for the formation of the regular satellites is that they have formed by two-body accretion in circumplanetary nebulae (see Stevenson, Harris, \& Lunine 1986 for a review, and Mosqueira \& Estrada 2001, 2002a, 2002b and Canup \& Ward 2002 for recent models), in much the same way as the planets are thought to have formed in the solar nebula. This model cannot be applied to the irregular satellites, because (1) they are, in general, well separated from the regular satellite systems, making it unlikely that they have formed from the same circumplanetary nebula; (2) their eccentricities, in general, are too large to have been the result of straightforward accretion; and most importantly, (3) most of them follow retrograde orbits, so they could not have formed in the same disk as the prograde regular satellites.

As a result, the irregular satellites are assumed to have been captured by planets from heliocentric orbits. However, their current orbits cannot result from a purely gravitational three-body, Sun-planet-satellite, capture because of the time-reversibility of Newton's laws of motion (i.e., if there is a path in, there must be a path out). ${ }^{8}$ If the capture hypothesis is correct, presumably some sort of dissipation process must have produced permanent captures. Three major scenarios for the formation of the irregular moons have been proposed; all are based on the idea of satellite capture from a heliocentric orbit.

1. A collisional scenario was originally proposed for the four prograde and four retrograde irregular satellites of Jupiter known before 2000. Moons in these groups have similar orbits and could have been formed in the collisional disruption of two parent bodies. Indeed, Colombo \& Franklin (1971) proposed that all eight satellites were formed by a single collision between an object temporarily captured in Jovian orbit and one in heliocentric orbit. Since the orbits of the prograde and retrograde Jovian groups are tightly clustered at largely distinct semimajor axis, however, a single collision is an unlikely scenario to form these groups. Thus, at least two collisions were needed. The collision scenario postulates that the observed groups of moons with similar orbital elements were formed by the same disruptive collision that assisted their capture.

2. Heppenheimer \& Porco (1977) proposed that retrograde irregular satellites were captured as the mass of the parent body grew via accretion. In this scenario, known as pull-down capture, the satellites were initially locked in the $1: 1$ mean motion resonance with their parent planet; as the mass of the planet grows, objects in some 1:1 resonant orbits can slowly transition to planetocentric orbits. A major weakness of this scenario, as Heppenheimer \& Porco proposed it, is that it does not produce the prograde irregular satellites.

3. Pollack, Burns, \& Tauber (1979) proposed that capture occurred when an object entered a circumplanetary gas envelope and decelerated via aerodynamic drag (and also, in some cases, disintegrated). However, this mechanism has the problem that once capture occurs, there is very little time (on the order of 10 years) before the object spirals into the planet. Pollack et al. circumvented this problem by suggesting that some $10^{4}, 100 \mathrm{~km}$ planetesimals were captured by

\footnotetext{
${ }^{8}$ It should be noted, however, that "long-lived" temporary captures can occasionally occur ( $\sim 100$ orbits; Kary \& Dones 1996).
}

and spiraled into Jupiter over the assumed $10^{5} \mathrm{yr}$ lifetime of the Jovian gas envelope. After some $10^{5} \mathrm{yr}$, the envelope contracts rapidly (in less than 10 years), and a few lucky survivors, the progenitors of today's Jovian irregulars, avoid being swept into the planet. While this scenario may actually work for Jupiter and Saturn, it is unclear whether it applies to Uranus and Neptune, because of their less massive gas envelopes (Pollack, Lunine, \& Tittemore 1991). Smaller fragments, being more strongly coupled to gas, should experience faster orbital evolution that spirals them in toward the planet and circularizes their orbits. This is contradicted by the much larger Phoebe being the closest of its cluster to Saturn; at Uranus, where Caliban and Sycorax, the largest irregular moons, are two of the three closest; at Jupiter, where satellites greatly differing in size have the same semimajor axis; and by the lack of any general correlation between satellite size and eccentricity (Gladman et al. 2001).

Once the irregular moons became bound to their parent planets, their planetocentric semimajor axes probably suffered some slow evolution, because of either the increasing mass of the planet, gas drag, or some other form of dissipation. This evolution is suggested by the fact that among the four major retrograde moons of Jupiter studied by Saha \& Tremaine (1993), Pasiphae and Sinope are located in a tiny portion of the orbital space characterized by the secular resonance $g-2 s+g_{5}=0$ (see also Whipple \& Shelus 1993). Here $g$ and $s$ are the apsidal and nodal frequencies of a moon and $g_{5}$ is the mean apsidal frequency of Jupiter. Saha \& Tremaine (1993) argued that the resonant capture of slowly evolving orbits is most likely responsible for this configuration (Henrard 1983).

Our study is motivated by the recent discoveries of 56 new irregular moons of the Jovian planets (Gladman et al. 1998, 1999, 2001; Sheppard et al. 2001, 2002). Together with the 10 irregular moons known previously, the total number of currently known irregulars is 66 (as of 2003 March 8). Our ultimate goal is to understand the origin of these bodies and what they can tell us about the formation of the Jovian planets. In this paper, however, we focus on the current physical and orbital characteristics of the irregular moons, such as their orbital stability, occurrence of resonances, and the fact that most irregular moons form groups of objects with similar orbits. These issues are fundamental for understanding the origin of the irregular satellites. In particular, we must discriminate between the observed properties of the satellite systems produced by the formation mechanism and those resulting from the later evolution.

In $\S 2$, we study the orbital stability of distant satellite orbits around Jovian planets by means of numerical integrations. In that section, we present the results of our lowresolution stability surveys. The purpose of these surveys is to find out whether the orbits of known irregular satellites bear some characteristics that can be related to their formation mechanism or whether they randomly sample the whole parameter space characterized by long-term orbital stability. In $\S 3$, we use a semianalytic model of the distant satellite orbits to explain the instabilities of the highly inclined orbits found in $\S 2$. In $\S 4$, we summarize the results of our highresolution surveys of the orbital stability and compare them with the orbital distribution of the discovered moons. In $\S 5$, we numerically integrate the orbits of 50 irregular moons of the Jovian planets for $10^{8} \mathrm{yr}$. The purpose of this integration 
is to see whether these orbits are stable over time intervals that are comparable to the age of the solar system, and to determine average orbits. We use the average orbits to classify irregular satellites into groups of bodies with similar orbits $(\S 6)$. Moreover, we calculate collision rates between pairs of irregular moons $(\S 7)$ and the effect of past collisional evolution that may have produced the populations of the irregular moons seen today $(\S 8)$. In $\S 9$, we systematically search for orbits of irregular satellites that lie within resonances. Our conclusions are given in $\S 10$.

We adopt the usual notation, denoting by $a, e, i, \varpi, \omega, \Omega$, $\lambda$, and $M$ the planetocentric semimajor axis, eccentricity, inclination, perihelion longitude and argument, node longitude, mean longitude, and mean anomaly of a satellite, and by $a_{j}, e_{j}, i_{j}, \varpi_{j}, \omega_{j}, \Omega_{j}, \lambda_{j}$, and $M_{j}$ the heliocentric elements of the $j$ th planet $(j=5,6,7,8$ for Jupiter, Saturn, Uranus, and Neptune, respectively; in some cases, we use letters J, S, U, and $\mathrm{N}$ for a subscript to indicate the respective planet). The ratio of the planetary masses $\left(m_{j}\right)$ to that of the Sun $\left(M_{\odot}\right)$ are denoted by $\mu_{j}\left(=m_{j} / M_{\odot}\right)$. The planetary orbital, perihelion, and nodal frequencies are denoted by $n_{j}, g_{j}$, and $s_{j}$, respectively; $n, g$, and $s$ are the same frequencies for the satellite's orbit. In a generic case, the planetary elements, masses, and frequencies are denoted by the subscript $p$. The universal gravitational constant is $\mathscr{G}$. If $M_{\odot}, \mathrm{AU}$, and the Julian year are used for units, $\mathscr{G}_{M_{\odot}}=(365.25 k)^{2}$, where $k=0.01720209895$ is the Gauss constant.

\section{LOW-RESOLUTION STABILITY SURVEYS}

Here we summarize the results of our stability surveys of distant satellite orbits. The idea was to numerically integrate a large number of test orbits for a long enough time interval to find locations where orbits are stable over long time spans. We compare these locations with the orbits of real satellites and draw constraints from this comparison on the formation mechanism of the irregular moons. Because we use a low-resolution grid for the initial $(a, e, i)$ of test orbits, we call this survey "the low-resolution survey" (our "high-resolution surveys" are discussed in $\S 4$ ).

We numerically integrated the orbits of the four Jovian planets and test satellites. The initial positions and velocities of the planets were obtained from the JPL DE405 ephemeris on JED 2,451,544.5 (2000 January 1). ${ }^{9}$ They were rotated to the invariable plane determined by the total angular momentum of the solar system and were corrected for the barycenter of the inner solar system. The masses of the terrestrial planets were then added to the mass of the Sun.

All test satellites were assumed massless with no effect on the other bodies in the integration. We integrated 2000 test satellites for each planet (1000 in prograde and 1000 in retrograde orbits). We used 10 test orbits with the same initial $(a, e, i)$ and with random initial $(\omega, \Omega, \lambda)$. The initial $(a, e, i)$ were distributed on a grid: $0.1 R_{j}^{\mathrm{H}} \leq a \leq 0.9 R_{j}^{\mathrm{H}}$, with a step $\Delta a=0.2 R_{j}^{\mathrm{H}}, 0 \leq e \leq 0.75(\Delta e=0.25)$, and $0^{\circ} \leq i \leq 180^{\circ}$ $\left(\Delta i=20^{\circ}\right)$. Here the inclination $i$ is referred to the planetary invariable plane.

We modified the second-order WHM integrator in Swift (Levison \& Duncan 1994) ${ }^{10}$ that uses the Wisdom-Holman map (Wisdom \& Holman 1991) so that the planets are

\footnotetext{
${ }^{9}$ See http://ssd.jpl.nasa.gov/eph_info.html.

${ }^{10}$ See http://www.boulder.swri.edu/ hal/swift.html.
}

integrated in the heliocentric frame and the satellites are integrated in the planetocentric frame. This choice is probably the best solution for minimizing the integration error of the symplectic scheme. We used a time step $(h)$ equal to $1 / 20$ of the orbital period of test satellites having $a=0.1 R^{\mathrm{H}}$ (the innermost orbits in the integration). This was about $0.011,0.027,0.077$, and $0.15 \mathrm{yr}$ for satellites of Jupiter, Saturn, Uranus and Neptune, respectively. The integration time span was $10^{6} \mathrm{yr}$.

We tested the precision of the integrator in the three-body Sun-Jupiter-moon system, where Jupiter's orbit was assumed to be circular. With this assumption, the Jacobi constant is an integral of motion (e.g., Murray \& Dermott 1999). The Jacobi constant with respect to the $j$ th planet is defined as

$$
C_{j}=\frac{V^{2}}{2}-\boldsymbol{n}_{j} \cdot(\boldsymbol{R} \times \boldsymbol{V})-G M_{\odot}\left(\frac{1}{R_{\odot}}+\frac{\mu_{j}}{R_{j}}\right),
$$

where $\boldsymbol{R}$ and $\boldsymbol{V}$ are the center-of-mass position and velocity vectors of a satellite, $R_{\odot}$ and $R_{j}$ are the satellite-Sun and satellite-planet distances, $\boldsymbol{n}_{j}$ is a vector perpendicular to the planet's orbital plane, and $\left\|\boldsymbol{n}_{j}\right\|=n_{j}$. We use this definition of the Jacobi constant, which formally corresponds to the orbital energy per unit mass of the test body in the reference system centered at the Sun-planet barycenter, and rotating with the angular speed and direction determined by $\boldsymbol{n}_{j}$; hence, it includes the effective centrifugal potential: $-\boldsymbol{n}_{j} \cdot(\boldsymbol{R} \times \boldsymbol{V})$. For the irregular moons of the $j$ th planet, $C_{j} \sim-3\left(n_{j} a_{j}\right)^{2} / 2$. For each integrated orbit of a test Jovian satellite $\left(0.1 R_{5}^{\mathrm{H}} \leq a \leq 0.9 R_{5}^{\mathrm{H}}\right)$, we compute the maximum fractional error produced by the integrator as $\delta C_{5}^{*}=\left[\max \left(C_{5}^{*}\right)-\min \left(C_{5}^{*}\right)\right] / C_{5,0}^{*}, \quad$ where $\quad C_{5}^{*}=C_{5}+$ $3\left(n_{5} a_{5}\right)^{2} / 2, C_{50}^{*}$ is the initial value, and $\max \left(C_{5}^{*}\right)$ and min $\left(C_{5}^{*}\right)$ are the maximum and minimum values reached by $C_{5}^{*}$ in the integration time span. With this definition, $\delta C_{5}^{*} \sim \delta a$, where $\delta a$ is the expected fractional error of the integrator in the satellite's semimajor axis (Hamilton \& Krivov 1997).

According to our tests, the fractional error $\delta C_{5}^{*}$ is nearly independent of $a$ if a fixed time step is used for orbits with $0.1 R_{j}^{\mathrm{H}} \leq a \leq 0.9 R_{j}^{\mathrm{H}}$. This was expected because at large $a$, the stronger solar perturbations that cause larger errors in the WHM scheme $\left(\propto a^{3}\right)$ are compensated by better sampling of orbital revolutions $\left(\propto n^{2} \propto a^{-3}\right.$ for the second-order WHM). With $h=0.011 \mathrm{yr}, \delta C_{5}^{*} \sim 10^{-4}$, which we consider satisfactory for our purposes. For comparison, the maximum expected error in the semimajor axis is about $1 / 10$ of Jupiter's diameter. Note that this apparent error can be largely reduced by the use of a symplectic corrector (Wisdom, Holman, \& Touma 1996) and that the intrinsic precision of the integrator is in fact much better. According to our tests, the fractional error $\delta C_{5}^{*}$ with $h=0.011 \mathrm{yr}$ is only a weak function of the eccentricity and inclination of the test orbit.

The planets and the Sun were assumed to be point masses. A test satellite was eliminated if its planetocentric distance was larger than 1 Hill radius (outer limit) or smaller than the semimajor axis of the outermost massive regular satellite (inner limit, $R^{\mathrm{in}}$ ). At planetocentric distances larger than the outer limit, the satellite is no longer gravitationally bound to its parent planet. At planetocentric distances smaller than the inner limit, the satellite crosses the orbit of a massive regular moon and suffers large orbital variations during 

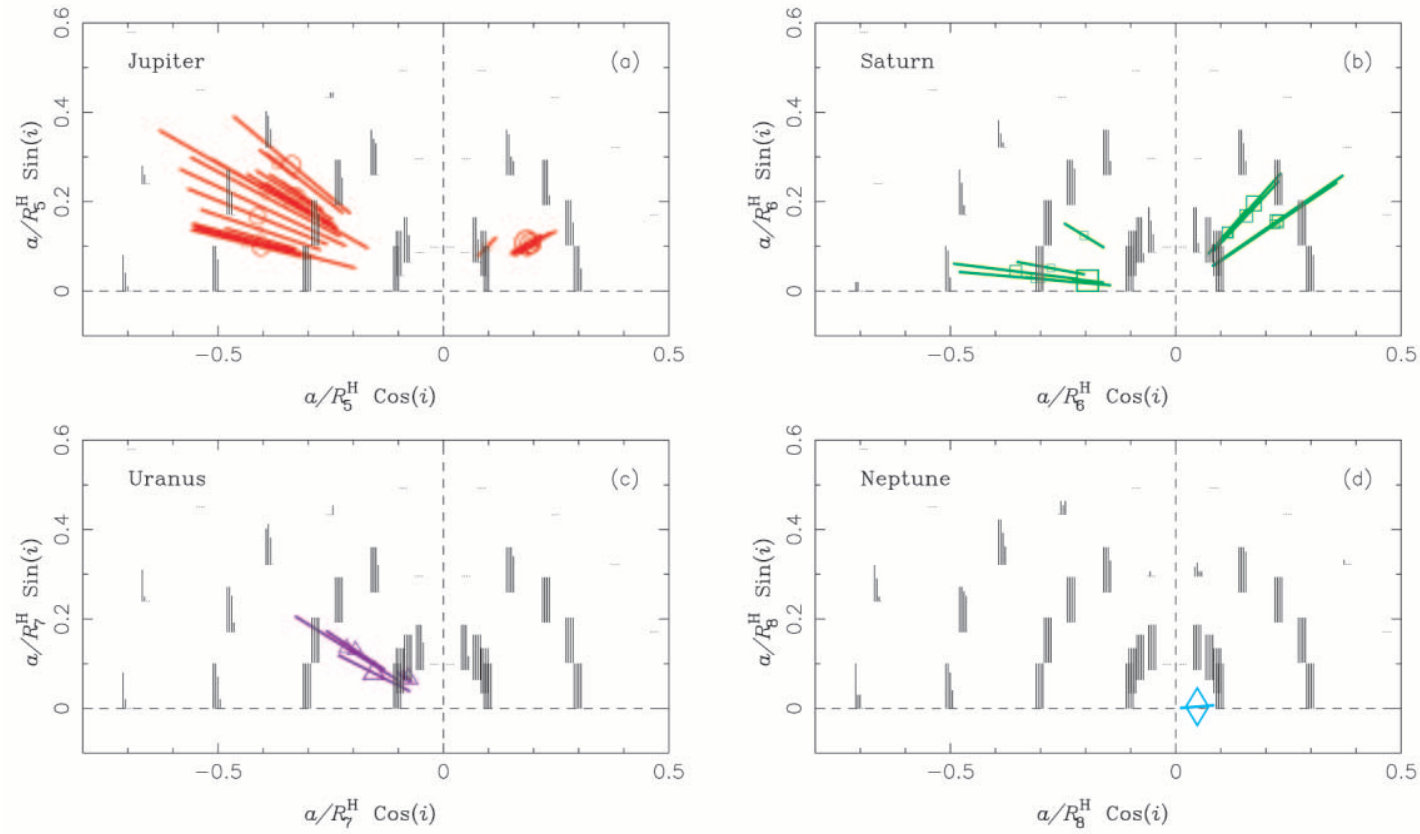

FIG. 2.- Results of the low-resolution surveys of the stability of the distant satellite orbits. There are four vertical line segments at each initial $a$ - and $i$-value whose lengths are proportional to the number of surviving test orbits at four eccentricity values $(0,0.25,0.5$, and 0.75 , from left to right $)$. The full length of 0.1 (in the scale of the $Y$-axis) means that all 10 integrated test orbits survived over $10^{6} \mathrm{yr}$. When no orbit survived with the initial ( $\left.a, e, i\right)$, a dot was plotted at the corresponding location. In the case of Saturn, we show the result with $R_{6}^{\text {in }}=0.00815 \mathrm{AU}$ (corresponding to Titan). This result does not greatly differ from the one using $R_{6}^{\text {in }}=0.0237 \mathrm{AU}$ (corresponding to Iapetus). With $R_{6}^{\text {in }}=0.0237 \mathrm{AU}$, however, most orbits with initial $i \sim 60^{\circ}$ were unstable, unlike in $(b)$, where most orbits at these inclinations were stable.

approaches to this object. We justify our choice of the physical model, removal criteria, and integration time span by a series of experiments. These experiments are explained at the end of this section.

For Jupiter, we chose $R_{5}^{\text {in }}=0.0125 \mathrm{AU}$, because the outermost massive satellite Callisto $(2403 \mathrm{~km}$ radius, $1076 \times 10^{20} \mathrm{~g}$ mass) has this semimajor axis. For Saturn, we ran two experiments with $R_{6}^{\text {in }}=0.00815 \quad \mathrm{AU}$ and $R_{6}^{\text {in }}=0.0237 \mathrm{AU}$, respectively. These limits correspond to the planetocentric distances of Titan $(2575 \mathrm{~km}$ radius, $1345.5 \times 10^{20} \mathrm{~g}$ mass) and Iapetus $(718 \mathrm{~km}$ radius, $15.9 \times 10^{20} \mathrm{~g}$ mass), respectively. For Uranus, $R_{7}^{\text {in }}=0.00389$ AU (Oberon, $761.4 \mathrm{~km}$ radius, $30.14 \times 10^{20}$ $\mathrm{g}$ mass). For Neptune, $R_{8}^{\text {in }}=0.00236 \mathrm{AU}$ (Triton, 1352.6 $\mathrm{km}$ radius, $214.7 \times 10^{20} \mathrm{~g}$ mass).

The results of our surveys are shown using a bar code in Figure 2. See the figure legend for the definition of the bar code. The pattern of stability in Figure 2 shows some common characteristics for all the Jovian planets, which we discuss in the following.

The test orbits started at $i \sim 90^{\circ}$ were unstable. Carruba et al. (2002b) showed that this instability occurs due to the so-called Kozai resonance (Kozai 1962). The Kozai resonance locks $\omega$ about $90^{\circ}$ or $270^{\circ}$ and causes coupled oscillations of $e$ and $i$. Consequently, the orbit's pericenter $q=a(1-e)$ can be driven to planetocentric distances smaller than $R^{\text {in }}$, when $e>e_{\text {crit }}=1-R^{\text {in }} / a$. If so, such an orbit is removed from the integration because it either impacts the planet, impacts a massive satellite, or is strongly perturbed and escapes from the Hill sphere (we justify this later). A simple illustration of how the Kozai resonance works is shown in Figure 3 for the test Jovian satellite orbits started at $a=0.1 R^{\mathrm{H}}, e=0.5$, and $i=40^{\circ}$. The effect of the Kozai resonance explains why no known irregular moon has $i \sim 90^{\circ}$ (Carruba et al. 2002b). We conclude that the relatively flat inclination distribution of the observed irregular moons does not constrain their formation mechanism.

There exists a certain asymmetry between the stability limits of prograde and retrograde orbits: the retrograde orbits are generally more stable at larger semimajor axes than the prograde orbits. This asymmetry also occurs in a simple three-body model where the planet is placed on a circular orbit. Since the Jacobi constant (eq. [1]) is conserved in this model, arguments have been proposed assuming $C=$ const (from more recent works, we cite Alvarellos 1996 and Hamilton \& Krivov 1997). Comparing the value of $C(a)$ of circular prograde orbits with the semimajor axis $a$ with $C(\mathrm{~L} 1)$, the value of the Jacobi constant at the Lagrangian point ${ }^{11} \mathrm{~L} 1$, Alvarellos (1996) argued that the prograde circular orbits become unbound for $a \gtrsim 0.48 R^{\mathrm{H}}$, because $C(a)>C(\mathrm{~L} 1)$ for these semimajor axes, which means the Jacobi constant does not confine planetocentric orbits. This turns out to be a good approximation of the semimajor axis limit for unstable orbits found numerically. Alvarellos also showed by means of numerical integrations that the initially circular retrograde orbits are stable at larger semimajor axes than the prograde orbits. According to his work, the instabilities of circular retrograde orbits occur for $a \gtrsim(0.72-$ $0.97) R^{\mathrm{H}}$. Unfortunately, Alvarellos was unable to derive this limit analytically, since for $0.25 R^{\mathrm{H}} \lesssim a \lesssim 0.72 R^{\mathrm{H}}$, all circular retrograde orbits are stable albeit having $C(a)>C(\mathrm{~L} 1)$.

Hamilton \& Krivov (1997) proposed that the asymmetry occurs due to different dynamics of prograde and retrograde

${ }^{11}$ Following Murray \& Dermott (1999), we call L1 the Lagrangian point located between the planet and the Sun. 


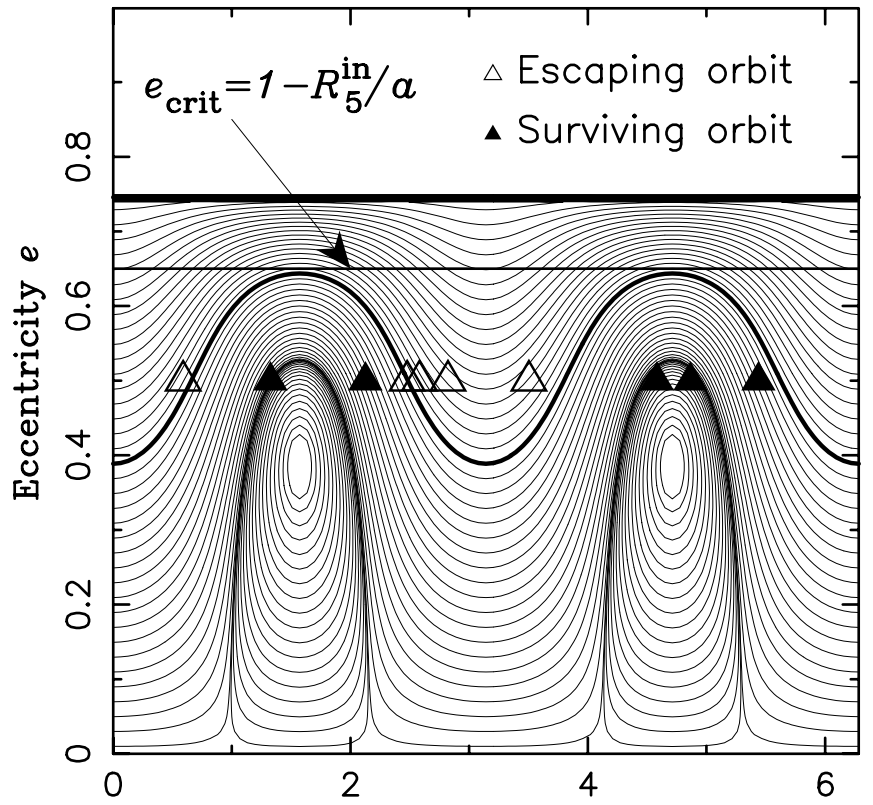

Argument of Perijove $\omega$

FIG. 3.-The evolution of $\omega$ and $e$ of the Jovian satellite orbits induced by solar tides. The trajectories were computed analytically using the averaged three-body model with Jupiter in a circular orbit around the Sun. In this model, $H^{*}=\left(1-e^{2}\right)^{1 / 2} \cos i$ is the integral of motion. We show trajectories for $H^{*}=0.575$. This value corresponds to the Jovian moons started with $e=0.5$ and $i=40^{\circ}$ (those with initial $a=0.0355 \mathrm{AU}$ are shown by triangles). For $e>e_{\text {crit }}=1-R_{5}^{\text {in }} / a \sim 0.65$, the orbits are Callisto-crossing because $q<R_{5}^{\text {in }}=0.0125$ AU when $\omega=90^{\circ}$ or $\omega=270^{\circ}$. The figure shows why some test orbits started at $a=0.0355 \mathrm{AU}, e=0.5$, and $i=40^{\circ}$ were eliminated in the stability surveys (open symbols), while other orbits, with the same initial $a, e$, and $i$, were long-lived (filled symbols). The initial value of the argument of perijove $\omega$ decided the fate. The orbits started with $\omega \sim 90^{\circ}$ or $\omega \sim 270^{\circ}$ never had $e>e_{\text {crit }}$. Thus, these orbits were long-lived because they never become Callisto-crossing during the cycle of $\omega$. Conversely, the orbits started with $\omega \sim 0^{\circ}$ or $\omega \sim 180^{\circ}$ were short-lived because they become Callisto-crossing when $\omega$ evolves to $\sim 90^{\circ}$ or $\sim 270^{\circ}$. The structure of trajectories seen in this figure is characteristic for the Kozai resonance (Kozai 1962)

orbits imposed by the Jacobi constant conservation. They analytically showed that the phase trajectory portraits of prograde satellites with $a \gtrsim 0.53 R^{\mathrm{H}}$ have a saddle point at $e=0$. Consequently, large eccentricities can be enforced at these semimajor axis. For retrograde orbits, Hamilton \& Krivov computed that the saddle point at $e=0$ occurs for $a \gtrsim 0.69 R^{\mathrm{H}}$, again in a good agreement with the instability limit found numerically.

To explain the asymmetry between prograde and retrograde satellites, we use the concept of the so-called evection resonance (e.g., Kaula \& Yoder 1976; Touma \& Wisdom 1998). The evection resonance occurs when the period of the precession of the satellite orbit's apsis and the period of the planet's orbital revolutions around the Sun are the same. Figure 4 shows the evolution of one test prograde satellite orbit started at Jupiter; initially, $a=0.5 R^{\mathrm{H}}=0.1775 \mathrm{AU}$, $e=i=M=0$. The initial semimajor axis is larger than the critical semimajor axis found for prograde orbits by Alvarellos (1996). Indeed, the orbit is unstable. The behavior of the angle $\varpi-\lambda_{5}$ shows that the orbit is affected by the evection resonance. This resonance is mathematically described by a "nonconvex" resonant normal form, which permits an indefinite growth of one of the actions ( $e$ goes
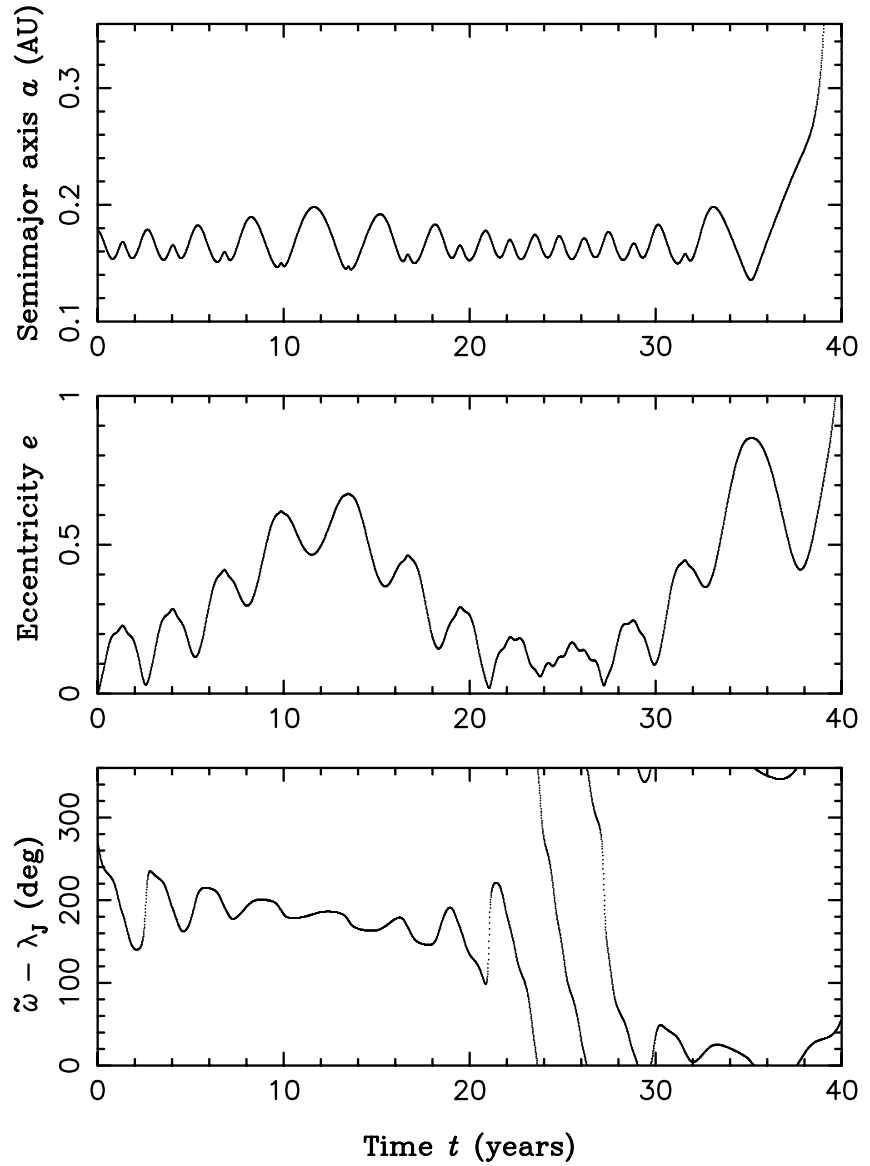

FIG. 4.-Evolution of the orbital elements of a test Jovian moon started in a circular, planar, and prograde orbit with $a=0.5 R_{5}^{\mathrm{H}} \sim 0.178 \mathrm{AU}$. The orbit of this moon was short-lived and became unbound at $t \sim 39 \mathrm{yr}$. The bottom panel shows the evolution of $\varpi-\lambda_{\mathrm{J}}$. This angle was nearly stationary for most of the evolution because of the effect of the evection resonance (e.g., Kaula \& Yoder 1976; Touma \& Wisdom 1998). The eccentricity increase up to $\sim 1$ - the cause of the eventual escape-was induced by resonant effects.

to 1). More intuitively, the resonance locks the orbit's apocenter to the apparent motion of the Sun around the parent planet. In this configuration, the effect of solar tides on a resonant moon accumulates at each aphelion passage of the moon, which causes an outward radial drift of its orbital apocenter; once close to the Hill sphere, the moon escapes. This is exactly what is seen in Figure 4 at $t \sim 40 \mathrm{yr}$ after the start of the integration. Other prograde test satellites started at $a=(0.5-0.9) R^{\mathrm{H}}$ behave similarly with progressively shorter escape times at larger initial $a$. For the retrograde orbits, the evection resonance occurs only at semimajor axis $a \gtrsim 0.7 R^{\mathrm{H}}$ because their periods of $\varpi$ are generally longer than those of prograde satellites (e.g., Saha \& Tremaine 1993).

What did we learn from the stability surveys about the irregular moons? (1) First of all, there is no major discrepancy between the current orbits of the irregular moons and the orbital locations characterized by stable satellite motion; the orbits of all known moons are located in the region characterized by long-term stability. (2) The prevalence of retrograde moons (36 retrograde vs. 14 prograde, as of 2002 August 16) may reflect the larger region of stability of the retrograde orbits. Indeed, some retrograde moons, if 
placed on prograde orbits with their $a$ and $e$ unchanged, would be dynamically short-lived. (3) No moons have nearpolar orbits and/or a very large semimajor axis, because of the destabilizing effects of the Kozai and evection resonances. (4) The lack of prograde moons of Uranus and the lack of retrograde moons of Neptune is not a consequence of the long-term instabilities of satellite orbits at these planets. Such moons, if formed at early epochs, would be dynamically long-lived (see end of this section).

Let us now explain our choice of the physical model, of the removal criteria, and of the integration time span. Concerning the physical model, we neglected the effect of the terrestrial planets. This choice is justified because their perturbations are on the order of $\mu_{j}\left(a / a_{p}\right)^{3}$, where $\mu_{j}$, $1 \leq j \leq 4$, is the mass of the $j$ th terrestrial planet divided by the mass of the Sun and $a / a_{p}$ is the ratio of the satellite's and the parent planet's semimajor axes (e.g., Kovalevsky \& Sagnier 1977). For example, the perturbations exerted by Earth on Jupiter's moon Pasiphae are less than $\sim 10^{-10}$ of the monopole field of Jupiter. We also neglected the effect of second and higher harmonics of the parent planet's gravitational potential and of the massive inner moons. To grossly account for the destabilizing effect of the massive inner moons on crossing orbits, we discarded those test satellites that reached distances $\leq R_{j}^{\text {in }}$, judging them unstable. To justify these choices, we performed additional experiments. We run stability surveys for the Jupiter irregular moons with the same initial orbits as before. We used, however, different integration setups in each case. (i) Instead of discarding a test satellite when its distance drops below the orbit of Callisto $(\approx 0.0125 \mathrm{AU})$, we removed it when the orbital perijove $q<70,000 \mathrm{~km} \sim 0.00047 \mathrm{AU}$, that is, when the orbit intersected the 1 bar Jupiter surface. (ii) The same as (i), but accounting for the second harmonic of the gravitational field of Jupiter and assuming that $J_{2}=14,735 \times 10^{-6}$ (Jacobson 2000). (iii) The same as (i), but including Ganymede and Callisto as massive bodies in the integration. We used the planetocentric version of the second-order symplectic integrator (Levison \& Duncan 1994), and a time step of $0.001 \mathrm{yr}(\sim 1 / 20$ of Ganymede's orbital period). In all these experiments, we compared patterns of stable/unstable orbits and the total number of discarded test bodies.

The difference between (iii) and the standard case (i.e., $q<0.0125 \mathrm{AU}$ ) is marginal. For prograde orbits, $78.7 \%$ of test bodies were discarded in the standard case $(55.1 \%$ outside the outer limit, $23.6 \%$ inside the inner limit), and $74.8 \%$ are discarded in experiment (iii) $(62.3 \%$ out, $9 \%$ in, $1.7 \%$ impact Ganymede, 1.8\% impact Callisto). For retrograde orbits, $68.5 \%$ were discarded in the standard case $(41.2 \%$ out, $27.3 \%$ in), and $64.3 \%$ in (iii) (49\% out, $7.9 \%$ in, $1.8 \%$ impact Ganymede, 5.6\% impact Callisto). The patterns of orbital stability in the standard and (iii) experiments were practically the same. We conclude that the Galilean satellites of Jupiter efficiently eliminate those irregular moons that reach $q<0.0125 \mathrm{AU}$ (see also Alvarellos et al. 2002). Similar roles are played by Titan/Iapetus, Oberon, and Triton for the satellite systems around Saturn, Uranus, and Neptune, respectively. Thus, it is justified to discard the test satellite orbits of planet $j$ when $q<R_{j}^{\text {in }}$.

It is interesting to note that including the $J_{2}$ of Jupiter slightly increases the number of discarded orbits. For example, there were $64.2 \%$ (59.1\% out, $5.1 \%$ in) discarded prograde orbits in $(i)$, and $70.9 \%(67 \%$ out, $3.9 \%$ in) discarded prograde orbits in (ii). Similarly, there were $55.1 \%$
$(49.2 \%$ out, $5.8 \%$ in) discarded retrograde orbits in $(i)$, and $60.7 \%(55.2 \%$ out, $5.5 \%$ in) discarded retrograde orbits in (ii). This occurred due to the smaller pericenter distances attained by orbits during the Kozai cycle when the oblateness effects were taken into account. Thus, in (ii), the orbits were unstable in a larger range of the initial $i$. Nevertheless, when $J_{2}$ was included in the experiments with the standard removal criteria $(q<0.0125$ AU in case of Jupiter), it caused only a small variation of the number of removed orbits. This justifies our choice of the physical model and the standard removal criteria used to produce Figure 2.

The integration time span (1 Myr) is short compared with the duration of the solar system $\left(\sim 4.6 \times 10^{9} \mathrm{yr}\right)$. One may thus wonder whether the stability of orbits over $1 \mathrm{Myr}$ is sufficiently indicative of the stability of orbits over the age of the solar system. Figure 5 shows the number of surviving test satellites at time $t$ observed in our standard experiments. This figure shows that the escape rate at $t \sim 10^{6} \mathrm{yr}$ is much smaller that the escape rate at previous times. This is especially clear for Jupiter, where only three moons escaped at $10^{5} \mathrm{yr}<t<10^{6} \mathrm{yr}$. Thus, for Jupiter, the stability pattern seen in Figure $2 a$ should not drastically change at later times. A similar result was obtained by Carruba et al. (2002b), who integrated a few hundred test orbits over $10^{9}$ yr. For other planets, a significant decay in the surviving satellite populations is observed at $10^{5} \mathrm{yr}<t<10^{6} \mathrm{yr}$. Thus, the

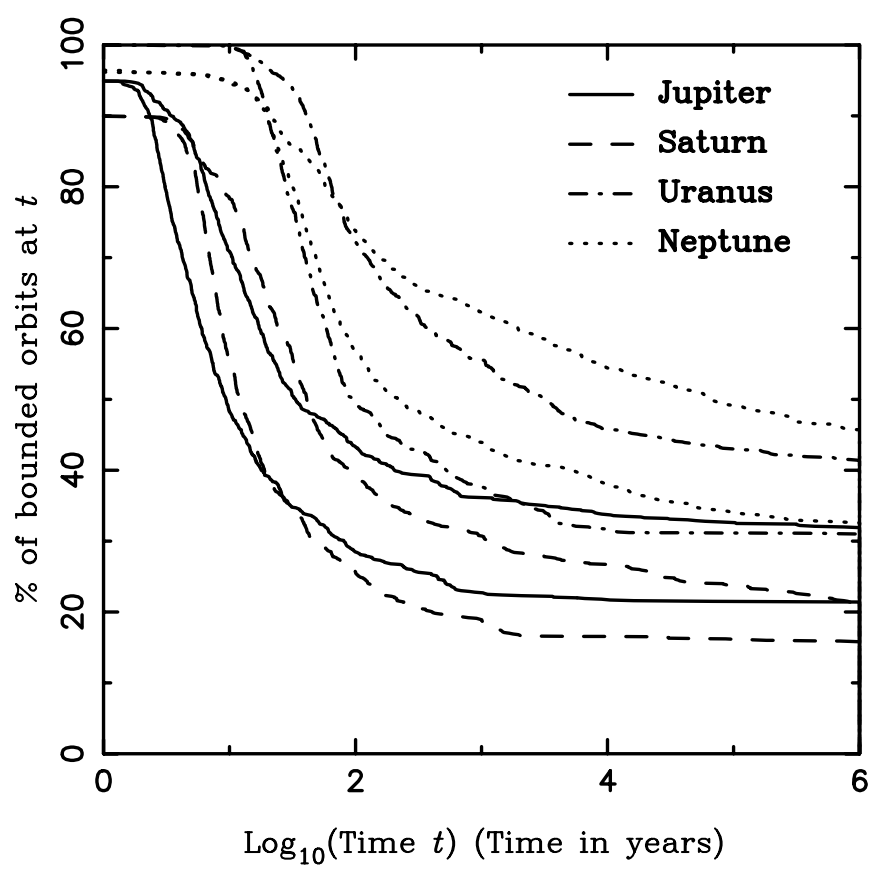

FIG. 5.-Fraction of bound orbits at time $t$ in our low-resolution surveys. Two curves per planet show this fraction for the prograde (bottom curve) and retrograde satellite orbits (top curve), respectively. Initially, all orbits were bound; large number of orbits became unbound in latter evolution. For all planets, the original population of the prograde satellite orbits was reduced by a larger factor than the population of the retrograde orbits. For Saturn, we used $R_{6}^{\text {in }}=0.0237 \mathrm{AU}$ here (corresponding to Iapetus). Ordered by the increasing fraction of bound satellite orbits at $t=10^{6} \mathrm{yr}$, the planets are Saturn, Jupiter, Uranus, and Neptune. If, however, $R_{6}^{\text {in }}=0.00815 \mathrm{AU}$ is used (corresponding to Titan), Jupiter becomes the planet with fewest survivors. The number of escape events per time interval is the largest at $t<100 \mathrm{yr}$. At $t>10^{5} \mathrm{yr}$, the escape rate is low. For example, only three satellites of Jupiter become unbound at $10^{5} \mathrm{yr}<t<10^{6} \mathrm{yr}$. 
stability regions shown in Figure 2 for these planets would shrink somewhat if longer time spans were used. The largest effect is expected for the retrograde satellites of Saturn and Neptune, where $\sim 3 \%$ and $\sim 4 \%$ of the initial orbits escape at $10^{5} \mathrm{yr} \leq t \leq 10^{6} \mathrm{yr}$. Extrapolating this trend to $4.6 \times 10^{9} \mathrm{yr}$, we estimate that some $\lesssim 12 \%$ and $\$ 16 \%$ additional escapes of retrograde orbits may occur at $10^{6} \mathrm{yr}<t<4.6 \times 10^{9} \mathrm{yr}$, respectively. We believe, however, that the main characteristic of the distribution of orbits stable over the age of the solar system may be already grasped from our $10^{6} \mathrm{yr}$ numerical integrations. We use longer integration time spans in our high-resolution surveys ( $\S 4)$.

\section{SEMIANALYTIC MODEL}

The orbital evolution of an irregular moon can be described by the Hamiltonian

$$
\mathscr{H}=-\frac{\mathscr{G}^{2} m_{j}^{2}}{2 L^{2}}+\mathscr{H}_{1}(L, G, H, l, g, h, t),
$$

where $\mathscr{H}_{1}(L, G, H, l, g, h, t)$ is obtained by writing the perturbation due to the Sun

$$
\mathscr{H}_{1}=-\mathscr{G}_{\odot}\left(\frac{1}{\Delta}-\frac{\boldsymbol{r} \cdot \boldsymbol{r}_{\odot}}{r_{\odot}^{3}}\right)
$$

in the Delaunay variables

$$
\begin{aligned}
L & =\sqrt{\mathscr{G} m_{j} a}, \quad l=M, \\
G & =L \sqrt{1-e^{2}}, \quad g=\omega, \\
H & =G \cos i, \quad h=\Omega .
\end{aligned}
$$

Here $\boldsymbol{r}$ and $\boldsymbol{r}_{\odot}$ are the planetocentric positions of the moon and the Sun, respectively, This model assumes that the Keplerian orbit of a massless satellite around its parent planet $j$ is perturbed solely by the Sun; direct effects of planets other than planet $j$ are neglected. We add the masses of the planets $i<j$ into the mass of the Sun $\left(M_{\odot}\right)$. Similarly, we add the masses of the massive inner satellites of the planet $j$ into $m_{j}$. The Hamiltonian (eq. [2]) is a good model for the perturbed motion of irregular satellites because solar tides are much larger than other perturbations. We evaluate equation (3) as a function of equation (4) numerically using the formalism described by Nesvorný et al. (2002b), or by using an expansion in powers of $a / a_{j} \ll 1$ (see Sidlichovský 1991).

Assuming that $a$ is nonresonant, we can average equation (3) over mean anomalies ( $l$ and $\left.l_{j}\right)$, neglect constant terms, and expand the resulting secular Hamiltonian in powers of $e_{j}$ and $i_{j}$. This gives

$$
\mathscr{H}_{\mathrm{sec}}=g_{j} \Lambda_{g_{j}}+s_{j} \Lambda_{s_{j}}+\sum_{n \geq 0} \mathscr{K}_{(n)}\left(G, H, g, h, \varpi_{j}^{*}, \Omega_{j}^{*}\right),
$$

where $\Lambda_{g_{j}}$ and $\Lambda_{s_{j}}$ are the canonical momenta that are conjugate to $\varpi_{j}^{*}=g_{j} t$ and $\Omega_{j}^{*}=s_{j} t$, respectively. Here $\mathscr{K}_{(n)}$ is of degree $n$ in $e_{j}$ and $i_{j}$. The leading term of this expansion is $\mathscr{K}_{(0)}$. This zeroth-order term is independent of the planetary eccentricities and inclinations, so that - by the d'Alembert rules - it is also independent of the planetary angles $\varpi_{j}^{*}$ and $\Omega_{j}^{*}$. Moreover, the d'Alembert rules also imply that $\mathscr{K}_{(0)}$ is independent of $h$ (i.e., of the longitude of the ascending node). We thus identify an integrable approximation of equation (3) that is usually called the Kozai Hamiltonian:

$$
\mathscr{H}_{\text {Kozai }}=\mathscr{K}_{(0)}(G, H, g)
$$

(Kozai 1962; Carruba et al. 2002b).

To order $\left(a / a_{j}\right)^{2}$, the Kozai Hamiltonian is

$$
\begin{aligned}
\mathscr{K}_{(0)}= & \frac{M_{\odot} n_{j}^{2}}{16\left(M_{\odot}+m_{j}\right)} a^{2}\left[\left(2+3 e^{2}\right)\left(3 \cos ^{2} i-1\right)\right. \\
& \left.+15 e^{2} \sin ^{2} i \cos 2 g\right]
\end{aligned}
$$

(see Kinoshita \& Nakai 1999). This expression is valid for every value of $e$ and $i$ but neglects terms of order higher than 2 in $a / a_{j}$. For irregular satellites, where $0.001 \lesssim a / a_{p} \lesssim$ 0.03 , equation (7) is nevertheless an excellent approximation. In the following, we study the dynamics described by equation (7).

Because equation (7) does not depend on $h$, the conjugate momentum $H \propto\left(1-e^{2}\right)^{1 / 2} \cos i$ is a constant of the motion. We show a dozen curves $H=$ const (six for prograde and six for retrograde orbits) in Figure 6. Because motion occurs on these curves, $e$ and $i$ are correlated: for prograde orbits $\left(0^{\circ} \leq i<90^{\circ}\right), e$ increases when $i$ decreases, and vice versa; for retrograde orbits $\left(90^{\circ} \leq i<180^{\circ}\right)$, $e$ increases when $i$ also increases, and vice versa. The maximum $e$ and $i$ that can be reached by an orbit with fixed $H$ are related through $e_{\max }=\sin i_{\max }$.

Figures 7 and 8 show the trajectories of equation (7) for six values of $H$. Figure 7 shows the trajectories parameterized by

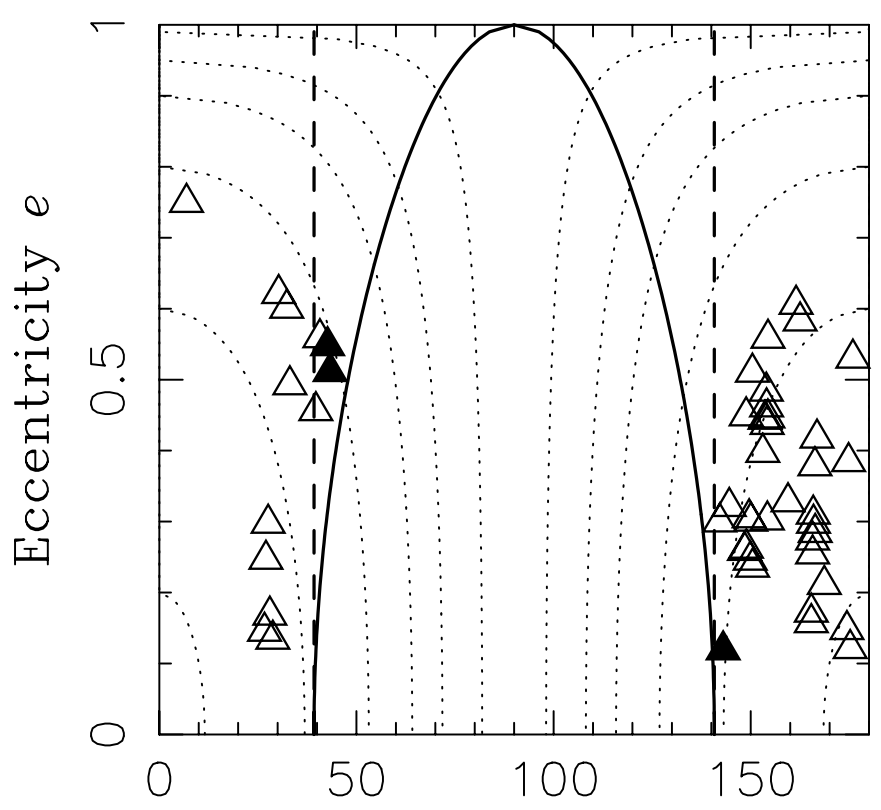

\section{Inclination $i(\mathrm{deg})$}

FIG. 6.-Loci of the $\omega$-libration (solid curves) and the limits of the last librating trajectories in $e$ and $i$ (dashed curves). The dotted lines are defined as $H=$ const, where six values of $H$ for both the prograde and retrograde orbits correspond to $e_{\max }=0.2,0.6,0.8,0.9,0.95$, and 0.99 . The phase portraits on these manifolds are shown in Figs. 7 and 8 . The orbital elements $(e, i)$ of 50 irregular moons are shown when $\omega \sim 90^{\circ}$ (triangles). The filled symbols are the orbits that show $\omega$ libration: S/2000 S5 and S/2000 S6 (prograde) and S/2001 J10 (retrograde). Orbits of these moons librate around $\omega=90^{\circ}$ (see also Fig. 5 in Carruba et al. 2002b). 

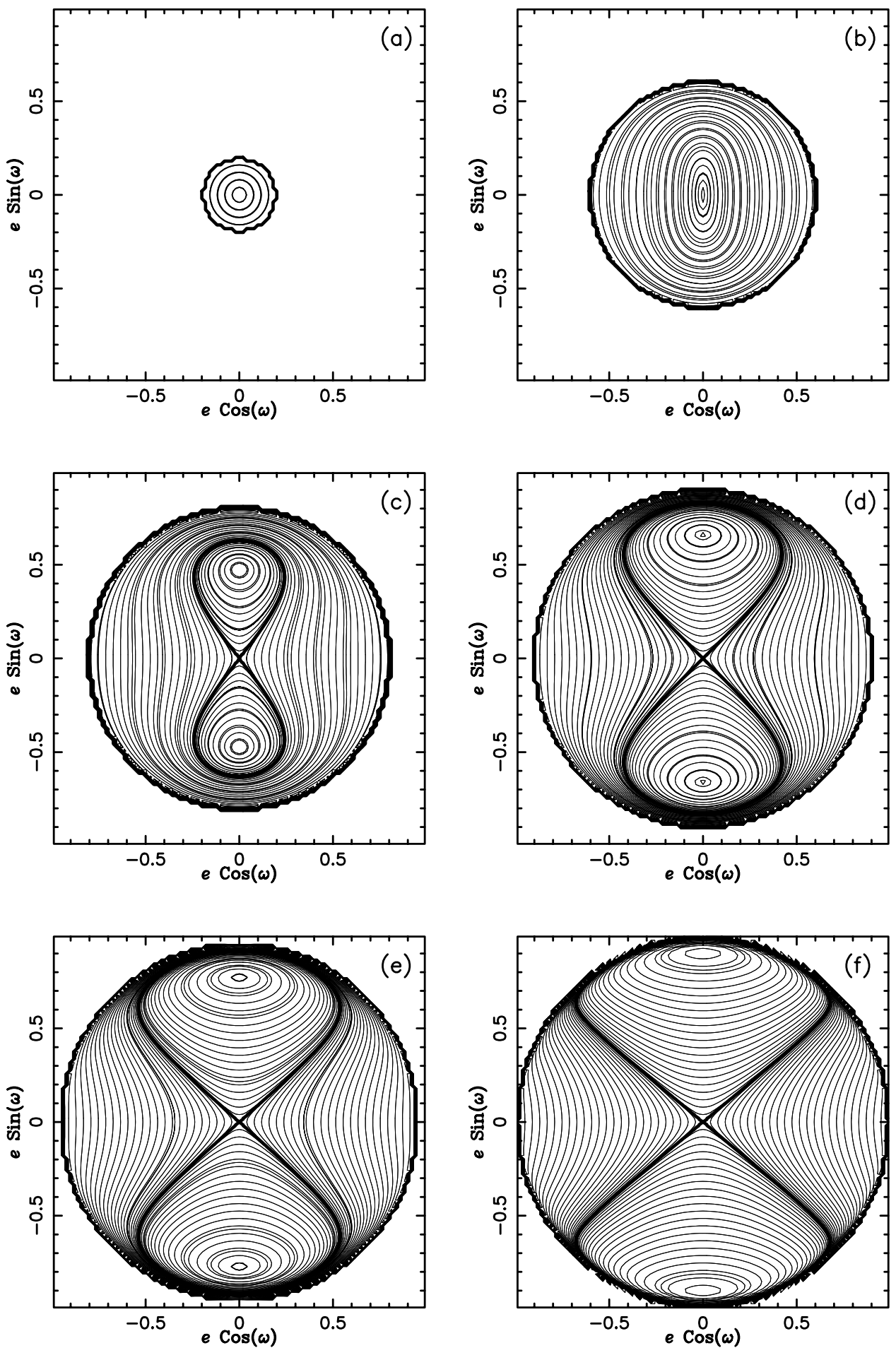

Fig. 7.-Evolution of $(e \cos \omega, e \sin \omega)$ for six values of $H=$ const. These values correspond to $e_{\max }=0.2,0.6,0.8,0.9,0.95$, and 0.99 (from $(a)$ to $\left.(f)\right)$. The thick line shaped like a figure eight separates librating and circulating trajectories. The dynamics seen in $(c)-(f)$ is characteristic for the Kozai resonance (see also Fig. 2 in Thomas \& Morbidelli 1996).

$(e \cos \omega, e \sin \omega)$, and Figure 8 shows trajectories in $(\sin i \cos \omega, \sin i \sin \omega)$. These dynamical portraits are independent of masses $m_{\odot}$ and $m_{j}$, and nearly independent of $a / a_{j}$. We have checked by computing the same phase portraits from the exact $\mathscr{K}_{(0)}(G, H, g)$ (using the doubleaveraging procedure described in Nesvorný et al. 2002b) that terms $\left(a / a_{j}\right)^{3}$ and higher in $\mathscr{K}_{(0)}(G, H, g)$ can be neglected for the orbital distances of the irregular satellites.
Libration of $\omega$ around $90^{\circ}$ or $270^{\circ}$ can occur for $\sin i>\left(\frac{2}{5}\right)^{1 / 2}$, that is, for $39^{\circ} .23<i<140^{\circ} 77$. The libration center is located at $\sin i=\left[\left(2+3 e^{2}\right) / 5\right]^{1 / 2}$. Figure 6 shows the minimum inclination of a librating orbit and the libration center. The maximum eccentricity reached by a trajectory starting at $e=0$ and $i_{0}$ is $e=\left[1-(5 / 3) \cos ^{2} i_{0}\right]^{1 / 2}$. If we assume that satellites that reach $a(1-e) \leq R_{j}^{\text {in }}$ are removed by the effect of the massive inner moons, we 

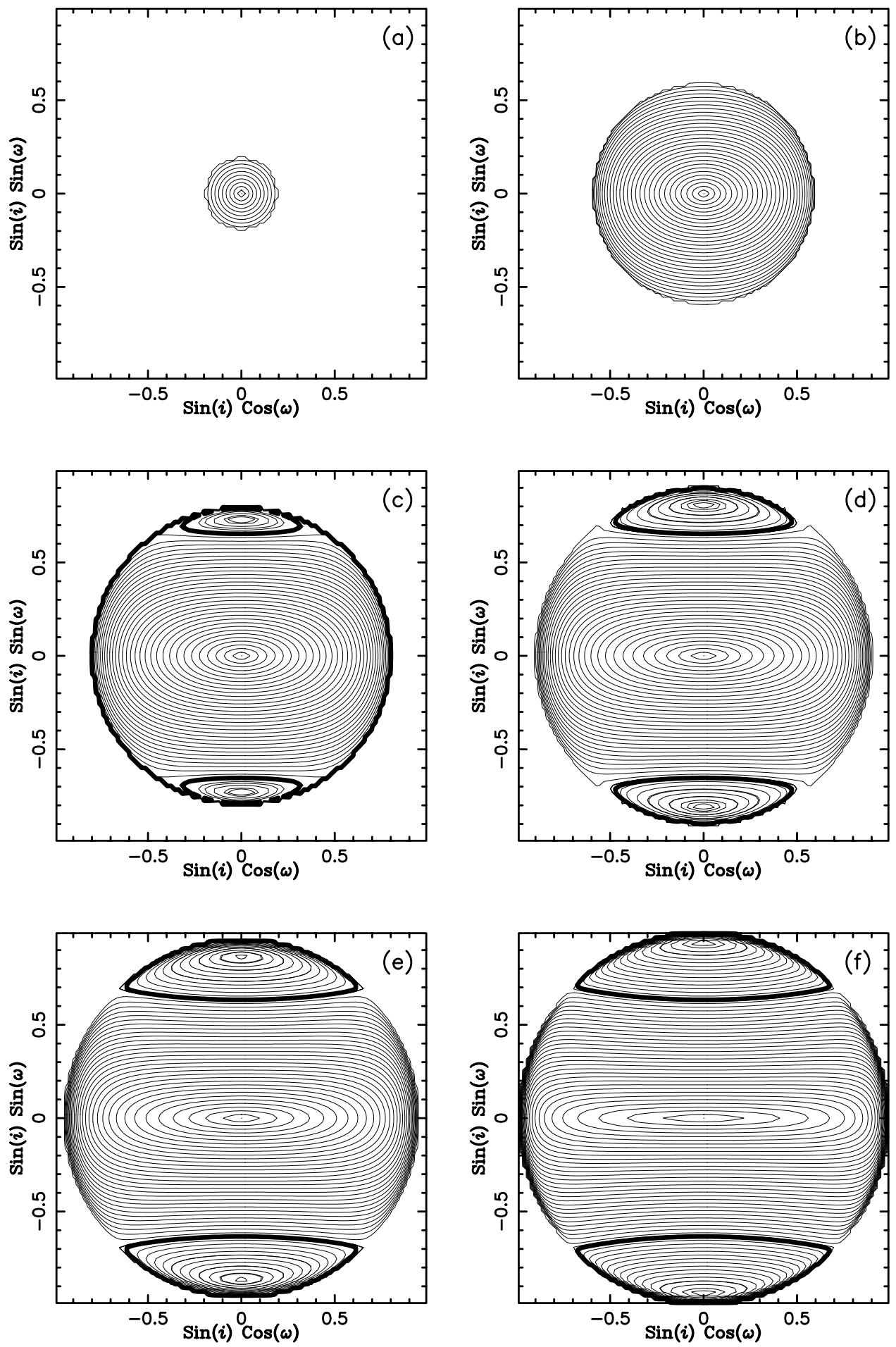
$(f))$.

calculate that this happens for

$$
i \geq \arccos \sqrt{\frac{2}{5}\left[1-\left(1-R_{j}^{\mathrm{in}} / a\right)^{2}\right]}
$$

(Gladman et al. 2001; Carruba et al. 2002b). We plot these limits in the upper panels of Figures 9-12 to compare them with the results of our high-resolution surveys (next section).
Figure 6 also shows the eccentricity and inclination of the orbits of the 50 known irregular moons when their $\omega \sim 90^{\circ}$. These values were obtained by a numerical integration of the exact equations of motion $(\S 5)$. Three satellites with $\omega$ librating orbits (S/2000 S5, S/2000 S6, and S/2001 J10) are within or close to the libration region in Figure 6. Other satellites that fall into the area between the solid and dashed lines do not actually move on $\omega$-librating trajectories, but 
follow a figure-eight trajectory just outside the separatrices of the libration island (see Fig. 7).

\section{HIGH-RESOLUTION STABILITY SURVEYS OF DISTANT SATELLITE ORBITS}

The integration setup of our high-resolution surveys was similar to that of the low-resolution surveys. We tracked, however, a larger number of orbits. Moreover, motivated by the results of the low-resolution surveys and of the analytic model, we selected the initial values of $\omega$ to properly account for the effect of the Kozai resonance. We performed two surveys per planet with $\omega=0^{\circ}$ in the first and $\omega=90^{\circ}$ in the second. According to Figure 3, the first choice corresponds to orbits with circulating $\omega$. The second choice, $\omega=90^{\circ}$, corresponds to orbits for which $\omega$ and $e$ oscillate around a fixed value. The maximum eccentricity attained by the orbits started with $\omega=90^{\circ}$ should be generally smaller than the maximum $e$ attained by orbits started with $\omega=0^{\circ}$ (Fig. 3). We thus expect different stability patterns depending on the initial value of $\omega$.

The other two angles, $\Omega$ and $\lambda$, were chosen at random between $0^{\circ}$ and $360^{\circ}$. As in the low-resolution surveys, we started orbits with $0.1 R_{j}^{\mathrm{H}} \leq a \leq R_{j}^{\mathrm{H}}$ and $0^{\circ} \leq i \leq 180^{\circ}$ for satellites of planet $j$. Here, however, we used a finer grid than in the low-resolution surveys. For Jupiter, we used $\delta a=0.01 R_{j}^{\mathrm{H}}$ and $\Delta i=2^{\circ}$ (a total of 8281 test orbits); for other planets, we used $\delta a=0.02 R_{j}^{\mathrm{H}}$ and $\Delta i=4^{\circ}$ (a total of 2116 test orbits). Moreover, we used four values of the initial eccentricity: $e=0,0.25,0.5$, and 0.75 . In total, more than 50,000 orbits were followed.

The coarser grid used for initial orbits in the case of Saturn, Uranus, and Neptune allowed us to reduce the CPU demand and to use longer integration time spans for these orbits. According to Figure 5, long integration time spans are required for the satellites of Saturn, Uranus, and Neptune to correctly account for the long-term instabilities. We used 0.5, 2, 6, and $10 \mathrm{Myr}$ integration time spans for the stability surveys of the distant satellite orbits around Jupiter, Saturn, Uranus, and Neptune, respectively.

Jupiter.-Figure 9 shows the results for Jupiter. The asymmetry between stable locations of prograde and retrograde orbits can readily be seen in this figure. At small eccentricities, the stability limit of the planar prograde orbits extends only up to $a \sim 0.4 R_{j}^{\mathrm{H}}$; conversely, the planar retrograde orbits are stable up to $a \sim 0.7 R_{j}^{\mathrm{H}}$. In $\S 3$, we explained that this asymmetry occurs due to the asymmetric location of the evection resonance.

Figure 9 also clearly shows the effect of the Kozai resonance on the orbital stability of Jovian irregular moons: most orbits with $i \sim 90^{\circ}$ are unstable due to large eccentricities enforced by this resonance $(\S 3)$. For the initially circular orbits of test satellites (Fig. 9, top), there is not much difference between the $\omega=0^{\circ}$ and $\omega=90^{\circ}$ plots, since the maxima attained by the eccentricities are the same (Fig. 7). We compute the limits of the stable orbits from $a\left(1-e_{\max }\right)>R_{5}^{\text {in }}$ using equation (8). The bold U-shaped curves in the top panels of Figure 9 show the largest (smallest) stable inclinations as a function of $a$ for prograde (retrograde) orbits. These limits are in good agreement with the numerical results.

For prograde orbits with $e \sim 0$, the largest stable initial inclination for $a \lesssim 0.4 R_{j}^{\mathrm{H}}$ is $\approx 60^{\circ}$. With $\omega=90^{\circ}$, this limiting inclination is nearly independent of the eccentricity for $e \lesssim 0.5$. For retrograde orbits with $e \sim 0$, the smallest stable initial inclination is $\approx 120^{\circ}$. With $\omega=90^{\circ}$, this limiting inclination increases to $\approx 130^{\circ}$ at $e \sim 0.5$. Similar results were published by Carruba et al. (2002b).

The stable satellite orbits form "wedges" centered at $(0,0)$ in Figure 9. The limits of these regions in $(a, i)$ can be empirically given in terms of simple functional forms. For small $e$, the prograde orbits with $i \lesssim 60^{\circ}$ and $a \lesssim 0.4 R_{j}^{\mathrm{H}}$, and the retrograde orbits with $i \gtrsim 120^{\circ}$ and $a \lesssim\left(0.4+0.3 i / 60^{\circ}\right) R_{j}^{\mathrm{H}}$ are stable. For prograde orbits, the semimajor axis range of stable orbits is roughly the same for all $e \lesssim 0.5$. For retrograde orbits, this range shrinks with increasing eccentricity. For example, the retrograde orbits started with $e=0.5$ and $\omega=0$ are stable if $i \gtrsim 130^{\circ}$ and $a \lesssim\left(0.4+0.1 i / 50^{\circ}\right) R_{j}^{\mathrm{H}}$. The angular and radial extents of the stability wedges are determined by the Kozai and evection resonances, respectively.

As expected, the orbits of all Jovian irregular satellites are located within the stable wedges. Only the orbits of Pasiphae and S/2000 J8, due to their higher eccentricities, are located somewhat close to the instability limit. We will show in $\S 5$ that the orbits of all Jovian irregulars, including Pasiphae and S/2000 J8, are long-lived.

Saturn.-The region of stable irregular satellite orbits of Saturn (Fig. 10) is somewhat smaller than that of Jupiter because $R_{6}^{\text {in }}>R_{5}^{\text {in }}$. For Saturn, we assume that $R_{6}^{\text {in }}=0.0237 \mathrm{AU}$ (roughly the semimajor axis of Iapetus), which is more than 2 times larger than the $R_{5}^{\text {in }}=0.0125$ AU used for Jupiter (the semimajor axis of Callisto). Is our value of $R_{6}^{\text {in }}$ justified? Iapetus is much smaller than Callisto, and it may not be large enough to effectively eliminate small bodies on crossing orbits. Although we were unable to precisely calculate the effect of Iapetus, some of our experiments suggest that this moon may indeed operate as an effective sink for the irregular moons that reach $\sim 0.0237$ AU. For example, the average lifetime against an impact on Iapetus of a $10 \mathrm{~km}$ diameter moon with $a=0.2 \mathrm{AU}, e=0.9$, and $i=30^{\circ}$ is about $500 \mathrm{Myr}$. Thus, if a comparable time interval is spent by a prograde irregular satellite in the high-eccentricity regime during the Kozai cycle, such a satellite is doomed to impact Iapetus. For a similar retrograde satellite (i.e., $a=0.2 \mathrm{AU}, e=0.9$, and $i=150^{\circ}$ ), the lifetime is only $\approx 180 \mathrm{Myr}$, that is, only $\approx 4 \%$ of the age of the solar system. Moreover, the gravitational scattering effect of Iapetus is probably more effective in removing the orbitcrossing population than collisions. We thus believe that our choice of $R_{6}^{\text {in }}$ is justified.

In our model with $R_{6}^{\text {in }}=0.0237 \mathrm{AU}$, the instabilities caused by the Kozai resonance show up at $60^{\circ} \lesssim i \lesssim 120^{\circ}$. For $\omega=0^{\circ}$ and $e \sim 0.5$, the range of unstable inclinations widens to $40^{\circ} \lesssim i \lesssim 140^{\circ}$. At $e \gtrsim 0.75$, all test distant satellite orbits are unstable.

Uranus and Neptune.-The pattern of stable/unstable orbits is similar for these planets (Figs. 11 and 12). The instabilities due to the Kozai resonance occur in a narrower inclination range $\left(80^{\circ} \lesssim i \lesssim 100^{\circ}\right.$ for $e=0$ and/or $\omega=90^{\circ}$ ) than for Jupiter and Saturn. This results from the very small planetocentric distances, at which Uranus's and Neptune's irregular moons start to interact with the massive inner moons (Oberon is at $R_{7}^{\text {in }}=0.00389 \mathrm{AU}$, Triton is at $\left.R_{8}^{\text {in }}=0.00236 \mathrm{AU}\right)$.

Another major difference with respect to Figures 9 and 10 is that many highly eccentric satellite orbits of Uranus and 

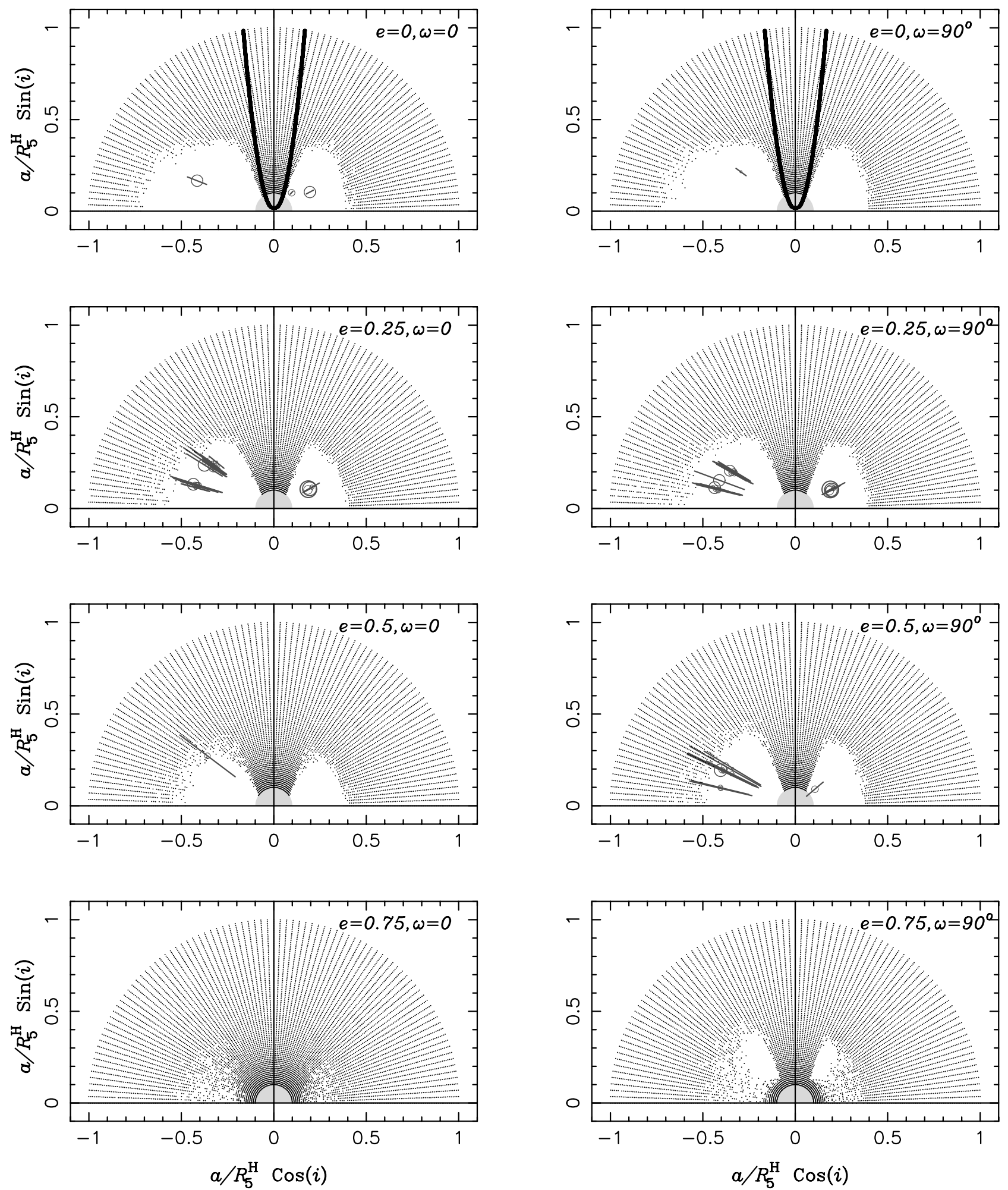

FIG. 9.-Results of the high-resolution surveys of the stability of distant satellite orbits of Jupiter. Test orbits with lifetimes shorter than $0.5 \mathrm{Myr}$ are denoted by dots; hence, blank regions represent stability. The gray semicircle at the origin shows the region not sampled by our initial conditions $\left(a<0.1 R_{J}^{\mathrm{H}}\right)$. In each panel, we also plot those known Jovian irregulars that have $\left|e(t)-e_{\text {panel }}\right| \leq 0.125$ when $\omega(t)=\omega_{\text {panel }}$ at time $t$, where $e_{\text {panel }}$ and $\omega_{\text {panel }}$ are the initial values of the surveyed test orbits shown in each panel (labels in upper right corners). For each irregular moon's orbit, we show the instantaneous elements at time $t$. These values were obtained by numerically integrating the orbits $(\S 5)$. The bold $U$-shaped curves in the top panels show the limits of stable inclinations calculated from eq. (8). Above these curves, the orbital perijove of an irregular moon drops down to the locations of the massive Jovian moons during the Kozai cycle. [See the electronic edition of the Journal for color versions of Figures 9-12.] 

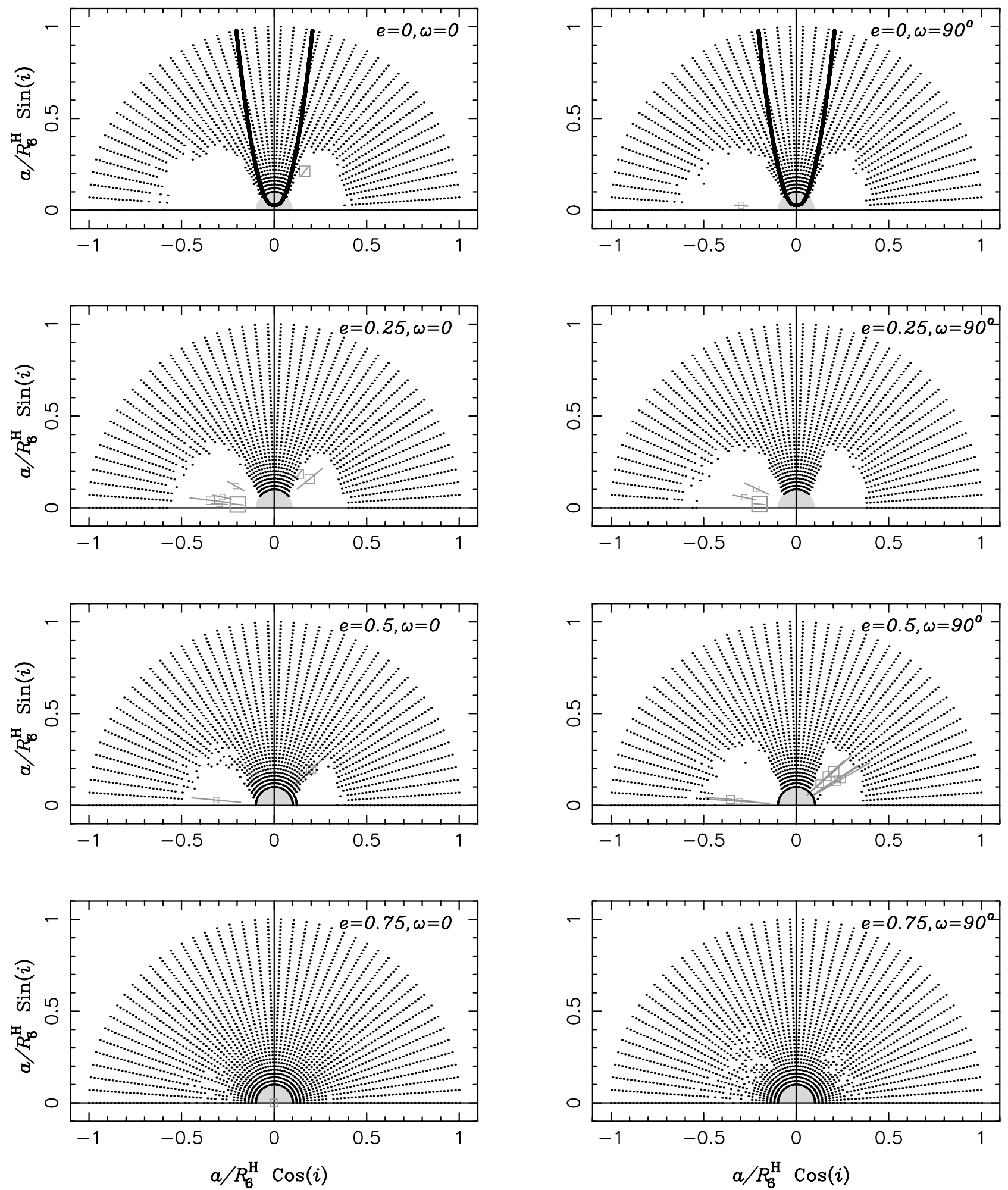

FIG. 10.-Results of the high-resolution surveys of the stability of distant satellite orbits of Saturn. Test orbits with lifetimes shorter than 2 Myr are denoted by dots. See legend of Fig. 9 .

Neptune are long-lived. These orbits are located in the wide blank regions in Figures 11 and 12 (bottom). Again, this occurs because of the small values of $R_{7}^{\text {in }}$ and $R_{8}^{\text {in }}$. Note that Neptune's irregular satellite Nereid, if placed on its current orbit about Jupiter, would be moving on a Callisto-crossing short-lived orbit. In general terms, this tells us that the orbital configurations of massive inner satellites and of the irregular moons are interrelated, not permitting crossing orbits during the Kozai cycle.

As a concluding remark for this section, we note that the regions of stable retrograde satellites in Figures 11 and 12 shrink with eccentricity (from $a \lesssim 0.7 R^{\mathrm{H}}$ at $e=0$ and $i \sim 180^{\circ}$ to $a \lesssim 0.4 R^{\mathrm{H}}$ at $e=0.75$ and $i \sim 180^{\circ}$ ), while the boundary of stable prograde orbits does not show the same 

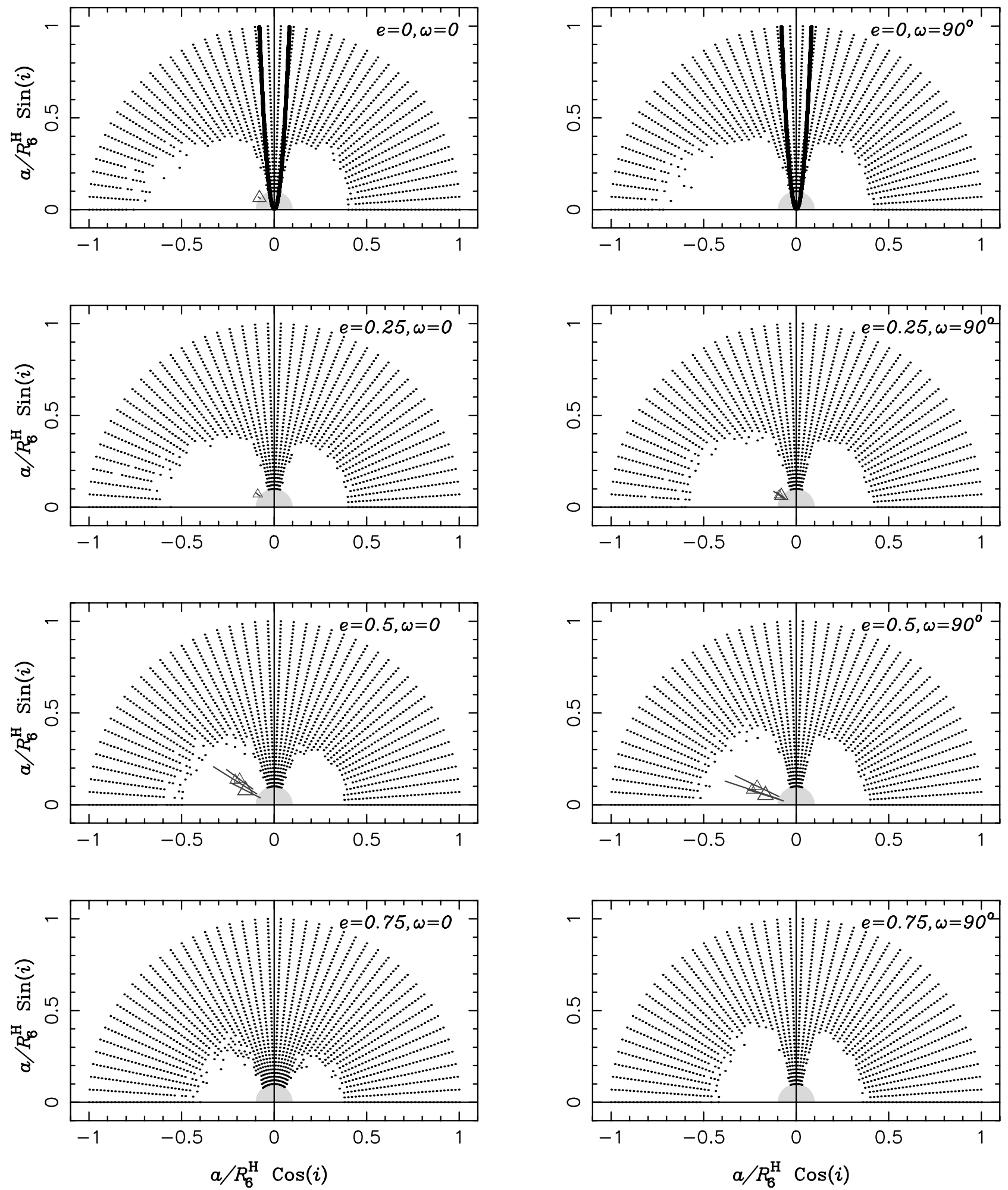

FIG. 11.-Results of the high-resolution surveys of the stability of distant satellite orbits of Uranus. Test orbits with lifetimes shorter than 6 Myr are denoted by dots. See legend of Fig. 9 .

behavior. We believe that the evection resonance, which becomes larger at larger $e$, is defining these patterns; it is conceivable that more eccentric orbits suffer stronger resonant-driven instabilities than the near-circular ones. Detailed calculations of this effect, however, are left for future studies.

\section{LONG-TERM EVOLUTION OF THE IRREGULAR MOONS}

We have numerically integrated for $10^{8} \mathrm{yr}$ the orbits of all presently known irregular satellites for which we have good initial orbits (50 orbits in total). Our motivation was to 

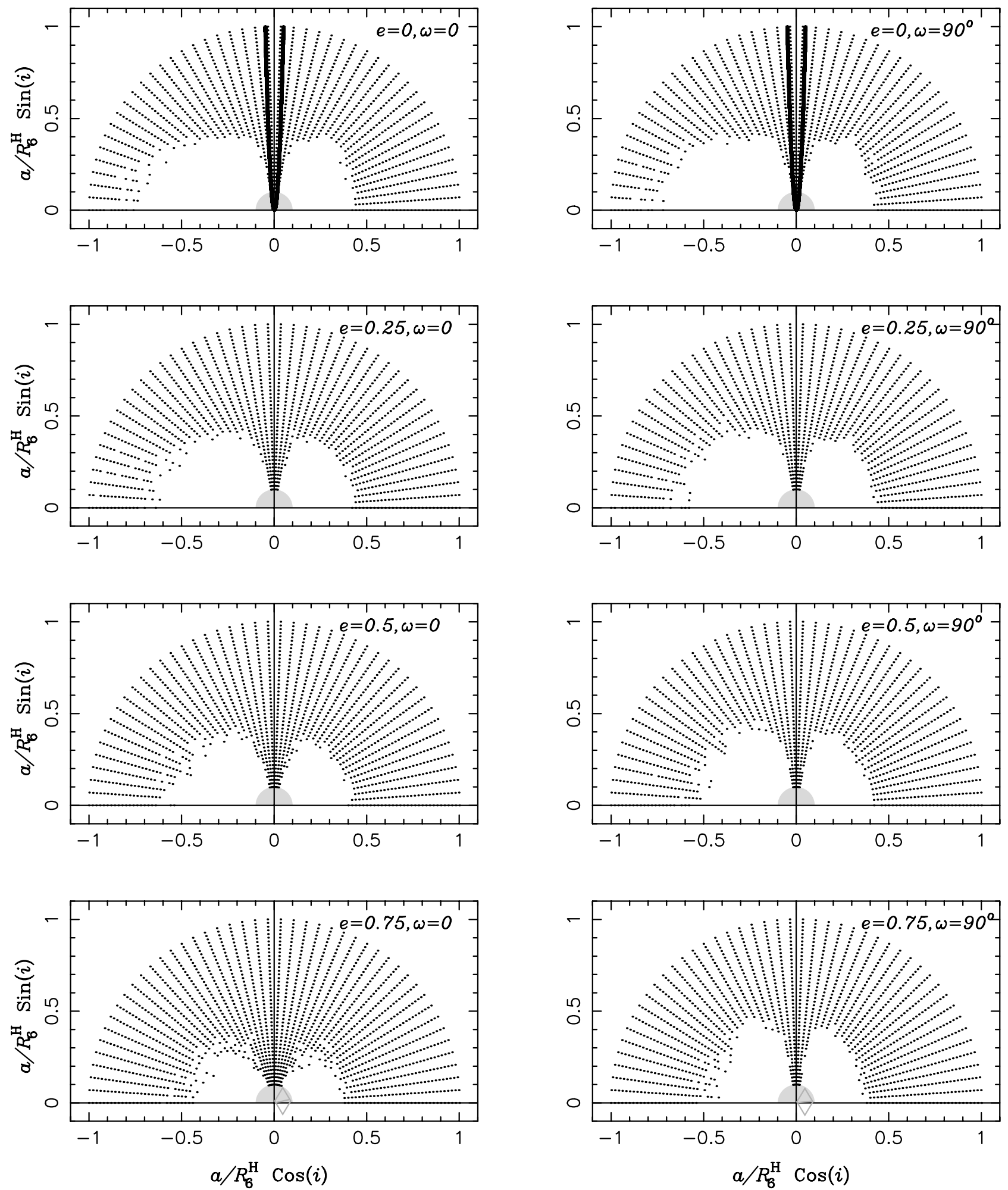

FIG. 12.- Results of the high-resolution surveys of the stability of distant satellite orbits of Neptune. Test orbits with lifetimes shorter than 10 Myr are denoted by dots. See legend of Fig. 9 .

determine (i) the average orbits of the moons, (ii) whether resonances occur, and (iii) whether the orbits of irregular moons are stable and/or show slow chaotic evolution over very long time spans.

The irregular satellites form several distinct dynamical groups with members of each group having similar orbits.
These groups were suggested to represent remnants of disrupted larger objects (Colombo \& Franklin 1971; Pollack et al. 1979; Gladman et al. 2001). The average orbital elements are useful in this context, because unlike the instantaneous orbital elements, they are more constant over long time spans. Thus, the average elements calculated in $(i)$ will 
be used to determine which irregular satellites have similar orbits, and thus may share a common origin.

Saha \& Tremaine (1993) showed that the orbits of Pasiphae and Sinope are affected by resonances. Since resonant orbits are rare if satellites form in random orbits and the orbits do not subsequently evolve, this result may suggest that some slow dissipative evolution of orbits of these satellites occurred in the past resulting in resonant captures (see Roy \& Ovenden 1954, 1955; Goldreich 1965; Murray \& Dermott 1999). In (ii), we will perform an analysis similar to that of Saha \& Tremaine for the 50 known irregular moons. If other resonances are identified, the case for some, probably primordial, orbital evolution will receive further support.

The irregular satellites have $a<0.5 R^{\mathrm{H}}$; it is hard to explain how their orbits could have originated recently, because significant energy dissipation would be needed to form them from one of the larger reservoirs of small bodies in the solar system. Thus, the irregular satellites are expected to be long-lived objects, and their origin probably dates back to the early stages of the formation of the solar system, when sources of dissipation were numerous (e.g., Pollack et al. 1979; Saha \& Tremaine 1993). We will verify for the first time whether this scenario is supported by the stability of the moons' orbits over time intervals comparable to the age of the solar system.

Our numerical model of the long-term evolution of the irregular satellites' orbits accounts for the gravitational perturbations from the Sun and four Jovian planets, which are assumed to be point masses. Effects of the parent planet's oblateness, relativistic corrections, and massive satellites were neglected. For irregular moons, these effects are smaller than $10^{-6}$ times the monopole field of the parent planet (Saha \& Tremaine 1993). We corrected planetary positions and velocities for the center of mass of the inner solar system and added the masses of the terrestrial planets into the Sun. Moreover, we corrected the satellite positions and velocities for the center of mass of the massive inner satellite systems and added the masses of inner moons into their parent planets.

The initial orbits of the planets and their satellites were obtained from the JPL Horizons Web page. ${ }^{12}$ For the moons of Jupiter, Uranus, and Neptune, we used the epoch 2000 as a starting date. Because the ephemerides of some of the irregular moons of Saturn were not available on this date, we used the epoch 2001 for the integration of the Saturn satellite system. The ephemerides were complete on this date. The precision of the initial orbital elements was poor for those moons that were discovered recently. We discuss later whether some of our conclusions may be affected by uncertainties in the initial orbits. The reference plane used for the numerical integration was the invariable plane of the solar system determined by its total angular momentum. The initial position and velocity vectors of all integrated bodies were rotated to this reference system.

Unlike for the stability surveys, we used a fourth-order symplectic integrator for the integration of the real bodies (Yoshida 1990; Wisdom \& Holman 1991). We made this choice because we required better integration precision here than the precision required for the numeri-

\footnotetext{
12 http://ssd.jpl.nasa.gov/horizons.html
}

cal surveys. According to our tests, the fourth-order symplectic integrator conserved the Jacobi constant better than the second-order integrator when time steps were adjusted in such a way that there were equal CPU demands in both cases. Typically, $\delta C^{*} \lesssim 10^{-7}$ for the time steps we used with the fourth-order method. We programmed the fourth-order symplectic scheme in mixed variables (fourth-order WHM; Wisdom \& Holman 1991) using the existing routines of Swift (Levison \& Duncan 1994). As before, we used the planetocentric reference system for satellites and the heliocentric reference system for planets. Moreover, we used a symplectic corrector in order to suppress short period variations of the orbital elements (Wisdom et al. 1996).

We used step sizes of 0.0178 and $0.0417 \mathrm{yr}$ for the inner and outer irregular satellites of Jupiter, respectively. The former value is about $1 / 20$ of the orbital period of the innermost prograde satellite; the latter value was used by Saha \& Tremaine (1993). We used step sizes of 0.0621, 0.0788, and $0.0495 \mathrm{yr}$ for satellites of Saturn, Uranus, and Neptune, respectively. These step sizes are $1 / 20$ of the orbital period of the innermost irregular satellite of these planets. The integration precision resulting from the selected step sizes is good enough for our purposes. All integrated satellite orbits remained bound over this interval. We will summarize and use the results of this simulation in the following sections.

\section{SATELLITE FAMILIES}

To determine which satellites have similar orbits and may thus share a common origin, we computed the average orbital elements of the 50 integrated orbits by averaging the osculating elements over $0 \mathrm{yr} \leq t \leq 10^{8} \mathrm{yr}$. The average values are listed in Tables 3 and 4 and are plotted in Figure 13. In a few cases, we numerically integrated the same orbit with two different time steps. This allowed us to estimate errors of the averaged elements resulting from the integration procedure. For Jupiter, J13 Leda-the second innermost Jovian irregular moon-was integrated with $h=0.0178 \mathrm{yr}($ standard $h)$ and $h=0.0089 \mathrm{yr}($ reduced $h)$ time steps. The differences of averaged orbital elements determined from the two runs were practically zero. Similarly, S/1999 J1 - the outermost Jovian irregular moonwas integrated with $h=0.0417 \mathrm{yr}$ (standard $h$ ) and $h=0.0178 \mathrm{yr}($ reduced $h)$ time steps. In this case, the maximum differences were $5 \times 10^{-6} \mathrm{AU}, 1.4 \times 10^{-3}$, and 0.03 for $a, e$, and $i$, respectively. This precision is satisfactory.

Figure 14 shows the osculating and average orbital elements for the retrograde group of irregular moons of Jupiter. This figure clearly demonstrates the usefulness of the averaged elements for the identification of dynamical groupings. The retrograde group looks like one, widely dispersed cluster in the space of the osculating orbital elements (Fig. 14, top). In the space of the average orbital elements, however, several much tighter clusters become apparent. We will discuss these and other dynamical structures in the following. We will adopt the terminology used in studies of asteroids and call groups of satellites with similar average elements satellite families. It is likely that at least some satellite families originated by collisional breakups of large precursor moons.

We use the Gauss equations to relate the size of a satellite family in the average orbital elements $(\delta a, \delta e, \delta i)$ with a 
TABLE 3

Average, Minimum, and Maximum over $10^{8}$ Years of the Orbital Elements of the Irregular Moons of Jupiter

\begin{tabular}{|c|c|c|c|c|c|c|c|c|c|c|}
\hline ID & Satellite & ave $(a)$ & $\min (a)$ & $\max (a)$ & ave $(e)$ & $\min (e)$ & $\max (e)$ & ave $(i)$ & $\min (i)$ & $\max (i)$ \\
\hline \multicolumn{11}{|c|}{ Prograde Group } \\
\hline $3 \ldots \ldots$ & J6 (Himalia) & 0.076428 & 0.0758 & 0.0771 & 0.15909 & 0.1098 & 0.2097 & 28.591 & 26.99 & 30.14 \\
\hline $4 \ldots \ldots \ldots \ldots . . .$. & J7 (Elara) & 0.078334 & 0.0777 & 0.0792 & 0.21262 & 0.1512 & 0.2765 & 28.048 & 26.08 & 29.96 \\
\hline $5 \ldots \ldots \ldots \ldots \ldots$ & J10 (Lysithea) & 0.078114 & 0.0775 & 0.0788 & 0.11581 & 0.0770 & 0.1549 & 27.634 & 26.24 & 28.97 \\
\hline $2 \ldots \ldots \ldots \ldots$ & J13 (Leda) & 0.074449 & 0.0739 & 0.0751 & 0.16327 & 0.1151 & 0.2126 & 28.075 & 26.50 & 29.61 \\
\hline $6 \ldots \ldots \ldots \ldots \ldots$ & $\mathrm{S} / 2000 \mathrm{~J} 11$ & 0.083526 & 0.0826 & 0.0847 & 0.24874 & 0.1741 & 0.3269 & 28.590 & 26.27 & 30.91 \\
\hline \multicolumn{11}{|c|}{ Ananke Family } \\
\hline $10 \ldots \ldots \ldots \ldots$ & J12 (Ananke) & 0.140674 & 0.1354 & 0.1470 & 0.24287 & 0.0653 & 0.4660 & 147.733 & 142.48 & 152.36 \\
\hline $7 \ldots \ldots \ldots \ldots \ldots$ & $\mathrm{S} / 2000 \mathrm{~J} 3$ & 0.139842 & 0.1347 & 0.1460 & 0.22378 & 0.0614 & 0.4282 & 148.676 & 143.94 & 152.82 \\
\hline $9 \ldots \ldots \ldots \ldots . .$. & $\mathrm{S} / 2000 \mathrm{~J} 5$ & 0.139732 & 0.1346 & 0.1459 & 0.23813 & 0.0641 & 0.4571 & 147.843 & 142.80 & 152.36 \\
\hline $8 \ldots \ldots \ldots \ldots . . .$. & $\mathrm{S} / 2000 \mathrm{~J} 7$ & 0.138719 & 0.1337 & 0.1446 & 0.23201 & 0.0425 & 0.4792 & 145.802 & 140.32 & 152.61 \\
\hline $24 \ldots \ldots \ldots \ldots$ & $\mathrm{S} / 2001 \mathrm{~J} 2$ & 0.140936 & 0.1356 & 0.1473 & 0.24123 & 0.0617 & 0.4654 & 147.643 & 142.37 & 152.27 \\
\hline $26 \ldots \ldots \ldots \ldots$ & $\mathrm{S} / 2001 \mathrm{~J} 3$ & 0.140764 & 0.1355 & 0.1471 & 0.22441 & 0.0689 & 0.4157 & 150.130 & 145.48 & 154.03 \\
\hline $25 \ldots \ldots \ldots \ldots$ & $\mathrm{S} / 2001 \mathrm{~J} 7$ & 0.139049 & 0.1340 & 0.1451 & 0.24114 & 0.0679 & 0.4572 & 148.018 & 142.82 & 152.52 \\
\hline \multicolumn{11}{|c|}{ Carme Family } \\
\hline 12. & J11 (Carme) & 0.154496 & 0.1473 & 0.1643 & 0.26329 & 0.1092 & 0.4258 & 164.530 & 160.93 & 167.03 \\
\hline $18 \ldots \ldots \ldots \ldots$ & $\mathrm{S} / 2000 \mathrm{~J} 2$ & 0.155906 & 0.1485 & 0.1659 & 0.25226 & 0.0991 & 0.4157 & 164.745 & 161.24 & 167.15 \\
\hline $14 \ldots \ldots \ldots \ldots$ & $\mathrm{S} / 2000 \mathrm{~J} 4$ & 0.153312 & 0.1463 & 0.1631 & 0.27764 & 0.1213 & 0.4418 & 164.395 & 160.62 & 166.98 \\
\hline $15 \ldots \ldots \ldots \ldots \ldots$ & $\mathrm{S} / 2000 \mathrm{~J} 6$ & 0.152432 & 0.1455 & 0.1619 & 0.27139 & 0.1188 & 0.4381 & 164.483 & 160.64 & 167.15 \\
\hline $13 \ldots \ldots \ldots \ldots$ & $\mathrm{S} / 2000 \mathrm{~J} 9$ & 0.154433 & 0.1472 & 0.1642 & 0.26006 & 0.1070 & 0.4221 & 164.785 & 161.19 & 167.19 \\
\hline $11 \ldots \ldots \ldots \ldots$ & $\mathrm{S} / 2000 \mathrm{~J} 10$ & 0.153363 & 0.1464 & 0.1628 & 0.25766 & 0.1087 & 0.4169 & 164.732 & 161.27 & 167.12 \\
\hline $27 \ldots \ldots \ldots \ldots$ & $\mathrm{S} / 2001 \mathrm{~J} 6$ & 0.152177 & 0.1454 & 0.1616 & 0.27217 & 0.1194 & 0.4347 & 164.580 & 160.91 & 167.07 \\
\hline $22 \ldots \ldots \ldots \ldots \ldots$ & $\mathrm{S} / 2001 \mathrm{~J} 8$ & 0.152934 & 0.1459 & 0.1625 & 0.27509 & 0.1213 & 0.4374 & 164.447 & 160.71 & 166.98 \\
\hline $29 \ldots \ldots \ldots \ldots \ldots$ & $\mathrm{S} / 2001 \mathrm{~J} 11$ & 0.155678 & 0.1483 & 0.1659 & 0.27134 & 0.1125 & 0.4381 & 164.711 & 161.04 & 167.26 \\
\hline \multicolumn{11}{|c|}{ Pasiphae and S/2000 J8 (?) } \\
\hline $16 \ldots \ldots \ldots \ldots \ldots$ & J8 (Pasiphae) & 0.156712 & 0.1484 & 0.1686 & 0.38708 & 0.1289 & 0.7115 & 148.429 & 138.10 & 155.81 \\
\hline $19 \ldots \ldots \ldots \ldots$ & $\mathrm{S} / 2000 \mathrm{~J} 8$ & 0.158126 & 0.1495 & 0.1707 & 0.42390 & 0.1700 & 0.6911 & 149.930 & 140.41 & 157.36 \\
\hline \multicolumn{11}{|c|}{$\mathrm{S} / 2001 \mathrm{~J} 5$ and S/2001 J1 (?) } \\
\hline $30 \ldots \ldots \ldots \ldots \ldots$ & $\mathrm{S} / 2001 \mathrm{~J} 5$ & 0.157404 & 0.1493 & 0.1686 & 0.33067 & 0.1059 & 0.5902 & 149.512 & 142.04 & 155.518 \\
\hline $31 \ldots \ldots \ldots \ldots \ldots$ & $\mathrm{S} / 2001 \mathrm{~J} 1$ & 0.159625 & 0.1511 & 0.1717 & 0.33219 & 0.1119 & 0.5948 & 150.643 & 143.01 & 156.575 \\
\hline \multicolumn{11}{|c|}{ Other } \\
\hline $1 \ldots \ldots \ldots \ldots \ldots$ & $\mathrm{S} / 1975 \mathrm{~J} 1$ & 0.049422 & 0.0493 & 0.0495 & 0.25130 & 0.0735 & 0.4654 & 44.409 & 39.31 & 47.68 \\
\hline $17 \ldots \ldots \ldots \ldots \ldots$ & J9 (Sinope) & 0.158110 & 0.1500 & 0.1697 & 0.29671 & 0.0771 & 0.5488 & 157.385 & 150.89 & 161.58 \\
\hline $20 \ldots \ldots \ldots \ldots$ & $\mathrm{S} / 1999 \mathrm{~J} 1$ & 0.158959 & 0.1506 & 0.1698 & 0.29398 & 0.0512 & 0.6036 & 145.131 & 137.93 & 151.92 \\
\hline $21 \ldots \ldots \ldots \ldots \ldots$ & $\mathrm{S} / 2001 \mathrm{~J} 10$ & 0.129176 & 0.1254 & 0.1330 & 0.14645 & 0.0613 & 0.2711 & 145.459 & 142.45 & 148.18 \\
\hline $23 \ldots \ldots \ldots \ldots \ldots$ & $\mathrm{S} / 2001 \mathrm{~J} 9$ & 0.139814 & 0.1345 & 0.1463 & 0.29397 & 0.0755 & 0.5731 & 144.323 & 137.80 & 150.88 \\
\hline $28 \ldots \ldots \ldots \ldots . .$. & $\mathrm{S} / 2001 \mathrm{~J} 4$ & 0.153579 & 0.1463 & 0.1631 & 0.28828 & 0.0843 & 0.5373 & 148.992 & 142.57 & 154.34 \\
\hline
\end{tabular}

Notes.-The columns are identification number (ID), name of the satellite, average, minimum, and maximum of the semimajor axis [ave (a), $\min (a)$, and $\max (a), a$ in $\mathrm{AU}$ ], average, minimum, and maximum of the eccentricity [ave $(e)$, min $(e)$, and $\max (e)$ ], and average, minimum, and maximum of the inclination [ave $(i), \min (i)$, and $\max (i), i$ in degrees with respect to the invariable plane of the solar system]. The satellites are grouped into clusters with similar average orbits.

selected velocity impulse $(\delta V)$ :

$$
\begin{aligned}
\frac{\delta a}{a} & =\frac{2}{n a \sqrt{1-e^{2}}}\left[(1+e \cos f) \delta V_{T}+(e \sin f) \delta V_{R}\right], \\
\delta e & =\frac{\sqrt{1-e^{2}}}{n a}\left[\frac{e+2 \cos f+e \cos ^{2} f}{1+e \cos f} \delta V_{\mathrm{T}}+(\sin f) \delta V_{R}\right], \\
\delta i & =\frac{\sqrt{1-e^{2}}}{n a} \frac{\cos (\omega+f)}{1+e \cos f} \delta V_{W} .
\end{aligned}
$$

Here $\delta V_{T}, \delta V_{R}$, and $\delta V_{W}$ are components of $\delta V$ along the direction of the orbital motion, in the radial direction, and perpendicular to the orbital plane, respectively. Assuming that a satellite family originated by a collisional disruption, $f$ and $\omega$ are the true anomaly and the perihelion argument of the disrupted parent body at the instant of the impact. If fragments are isotropically ejected from the breakup site with velocities $V_{\text {ejc }}$ exceeding the escape velocity $V_{\text {esc }}$ by $\delta V=\left(V_{\text {ejc }}^{2}-V_{\text {esc }}^{2}\right)^{1 / 2}<V_{\max }$, equation (9) shows that their osculating orbital elements will be located within an ellipsoid centered at the parent body's initial- $(a, e, i)$ orbit. The shape and orientation of the ellipsoid are determined by $V_{\max }, f$, and $\omega$. The same structure is conserved by the transformation from the osculating to proper elements.

Jupiter.-The prograde group of Jovian irregular moons is more compact than the retrograde group (Fig. 13). 
TABLE 4

Average, Minimum, and Maximum over $10^{8}$ Years of the Orbital Elements of the Irregular Moons of Saturn, Uranus, and Neptune

\begin{tabular}{|c|c|c|c|c|c|c|c|c|c|c|}
\hline ID & Satellite & ave $(a)$ & $\min (a)$ & $\max (a)$ & ave $(e)$ & $\min (e)$ & $\max (e)$ & ave $(i)$ & $\min (i)$ & $\max (i)$ \\
\hline \multicolumn{11}{|c|}{ First Inclination Group } \\
\hline$\ldots \ldots \ldots$ & $\mathrm{S} / 2000 \mathrm{~S} 4$ & 0.12126 & 0.1179 & 0.1256 & 0.51777 & 0.3394 & 0.7149 & 38.0703 & 28.953 & 46.98 \\
\hline 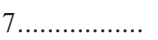 & $\mathrm{S} / 2000 \mathrm{~S} 10$ & 0.117059 & 0.1145 & 0.1204 & 0.46900 & 0.3070 & 0.6482 & 37.4936 & 29.942 & 45.02 \\
\hline $8 \ldots \ldots \ldots \ldots \ldots \ldots$ & $\mathrm{S} / 2000 \mathrm{~S} 11$ & 0.109487 & 0.1073 & 0.1121 & 0.49066 & 0.3199 & 0.6913 & 37.4598 & 29.233 & 45.80 \\
\hline \multicolumn{11}{|c|}{ Second Inclination Group } \\
\hline ...... & $\mathrm{S} / 2000 \mathrm{~S} 2$ & 0.10035 & 0.0990 & 0.1018 & 0.34628 & 0.1091 & 0.6563 & 49.2317 & 39.62 & 55.36 \\
\hline $6 \ldots \ldots \ldots \ldots \ldots . .$. & $\mathrm{S} / 2000 \mathrm{~S} 3$ & 0.117386 & 0.1150 & 0.1201 & 0.31801 & 0.0705 & 0.6073 & 47.7310 & 39.83 & 53.68 \\
\hline 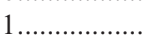 & $\mathrm{S} / 2000 \mathrm{~S} 5$ & 0.0755614 & 0.0752 & 0.0760 & 0.30824 & 0.0945 & 0.5713 & 47.8947 & 40.33 & 52.68 \\
\hline $2 \ldots \ldots \ldots \ldots \ldots \ldots$ & $\mathrm{S} / 2000 \mathrm{~S} 6$ & 0.0759147 & 0.0755 & 0.0763 & 0.30272 & 0.0940 & 0.5733 & 47.9994 & 40.40 & 52.69 \\
\hline \multicolumn{11}{|c|}{ Phoebe Group (?) } \\
\hline 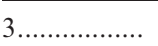 & Phoebe & 0.0864781 & 0.0860 & 0.0870 & 0.16426 & 0.1390 & 0.1910 & 175.179 & 173.97 & 176.44 \\
\hline $13 \ldots \ldots \ldots \ldots \ldots$ & $\mathrm{S} / 2000 \mathrm{~S} 1$ & 0.153335 & 0.1488 & 0.1596 & 0.33684 & 0.2222 & 0.4541 & 173.064 & 170.61 & 175.10 \\
\hline $12 \ldots \ldots \ldots \ldots \ldots$ & $\mathrm{S} / 2000 \mathrm{~S} 7$ & 0.135534 & 0.1324 & 0.1400 & 0.47092 & 0.3587 & 0.5765 & 175.563 & 173.31 & 177.51 \\
\hline $10 \ldots \ldots \ldots \ldots \ldots$ & $\mathrm{S} / 2000 \mathrm{~S} 9$ & 0.124208 & 0.1218 & 0.1267 & 0.20792 & 0.1443 & 0.2790 & 167.139 & 164.92 & 169.11 \\
\hline $11 \ldots \ldots \ldots \ldots \ldots$ & $\mathrm{S} / 2000 \mathrm{~S} 12$ & 0.129377 & 0.1272 & 0.1319 & 0.11547 & 0.0707 & 0.1654 & 176.048 & 174.82 & 177.33 \\
\hline \multicolumn{11}{|c|}{ S/2000 S8 Alone } \\
\hline $5 \ldots \ldots \ldots \ldots \ldots . .$. & $\mathrm{S} / 2000 \mathrm{~S} 8$ & 0.104117 & 0.1030 & 0.1054 & 0.27315 & 0.1746 & 0.3826 & 152.007 & 148.17 & 155.61 \\
\hline \multicolumn{11}{|c|}{ Caliban and Stephano (?) } \\
\hline 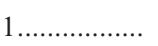 & Caliban & 0.047900 & 0.0479 & 0.0479 & 0.19215 & 0.0700 & 0.3173 & 141.185 & 138.31 & 144.60 \\
\hline 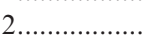 & Stephano & 0.053133 & 0.0531 & 0.0532 & 0.23253 & 0.1233 & 0.3418 & 143.460 & 140.23 & 147.04 \\
\hline \multicolumn{11}{|c|}{ Other } \\
\hline 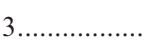 & Sycorax & 0.081501 & 0.0811 & 0.0820 & 0.51967 & 0.4410 & 0.6027 & 156.932 & 150.88 & 162.92 \\
\hline 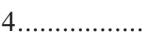 & Prospero & 0.109518 & 0.1084 & 0.1109 & 0.43781 & 0.3057 & 0.5833 & 149.323 & 142.53 & 156.15 \\
\hline $5 \ldots \ldots \ldots \ldots \ldots$ & Setebos & 0.117106 & 0.1154 & 0.1192 & 0.57755 & 0.4494 & 0.7085 & 153.575 & 144.42 & 162.10 \\
\hline \multicolumn{11}{|c|}{ Nereid } \\
\hline 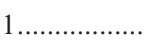 & Nereid & 0.03690 & 0.0369 & 0.0369 & 0.74602 & 0.7350 & 0.7571 & 9.663 & 5.368 & 13.474 \\
\hline
\end{tabular}

Note.-The columns are the same as in Table 3.

Assuming that these groups were formed by two collisional breakups, this would mean that the collision that formed the prograde group was less energetic than the one that formed the retrograde group (Colombo \& Franklin 1971). We calculate from Gauss's equations that $50 \mathrm{~m}$ $\mathrm{s}^{-1} \lesssim \delta V \lesssim 400 \mathrm{~m} \mathrm{~s}^{-1}$ for the prograde group, and $300 \mathrm{~m}$ $\mathrm{s}^{-1} \lesssim \delta V \lesssim 500 \mathrm{~m} \mathrm{~s}^{-1}$ for the retrograde group. Curiously, both these velocity ranges (especially the one for the retrograde group) are inconsistent with the velocity dispersion of multi-kilometer collisional fragments derived for catastrophic collisions by other means. For example, laboratory impact experiments, where centimeter-sized projectiles are shot into targets, and numerical hydrocode experiments, which are capable of simulating hypervelocity collisions among large bodies, both indicate that mean and median ejection velocities from impacts are on the order of several times $10 \mathrm{~m} \mathrm{~s}^{-1}$ (Benz \& Asphaug 1999; Michel et al. 2001, 2002). Whenever high velocities are obtained in hydrocode models, the fragment distribution is dominated by small and thus unobservable fragments (e.g., Pisani, Dell'Oro, \& Paolicchi 1999). Though it is possible that hydrocode results are inaccurate in these circumstances, their results have been substantiated using both laboratory impact experiments and underground nuclear explosions (e.g., Benz \& Asphaug 1999).

We are thus left with a contradiction: either we invoke some mechanism that further disperses orbits in addition to the velocity spread expected from their formation, or we should reject a simple scenario for the collisional origin of the two satellite groups. A closer inspection of the retrograde satellite group shows that there seem to exist several subclusters (Fig. 14). The average orbits of eight satellites, $\mathrm{S} / 2000 \mathrm{~J} 2, \mathrm{~J} 4, \mathrm{~J} 6$, J9, and $\mathrm{J} 10$ and $\mathrm{S} / 2001 \mathrm{~J} 6$, J8, and $\mathrm{J} 11$, cluster tightly around the average orbit of J11 Carme, a 40 $\mathrm{km}$ diameter moon; six satellites, $\mathrm{S} / 2000 \mathrm{~J} 3$, J5, and $\mathrm{J} 7$ and $\mathrm{S} / 2001 \mathrm{~J} 2, \mathrm{~J} 3$, and J7, have orbits similar to J12 Ananke, a $30 \mathrm{~km}$ diameter moon. Moreover, the satellite S/2000 J8 may be part of the group related to Pasiphae. ${ }^{13}$ If we use Gauss's equations to compute $\delta V$ for these groups, we find 5 $\mathrm{m} \mathrm{s}^{-1} \lesssim \delta V \lesssim 50 \mathrm{~m} \mathrm{~s}^{-1}$ for the group of Carme (which we call the Carme family) and $15 \mathrm{~m} \mathrm{~s}^{-1} \lesssim \delta V \lesssim 80 \mathrm{~m} \mathrm{~s}^{-1}$ for

\footnotetext{
${ }^{13}$ From the recently discovered moons of Jupiter (not integrated here) $\mathrm{S} / 2003 \mathrm{~J} 1, \mathrm{~J} 5, \mathrm{~J}$, J10, and J11 may be members of the cluster around J11 Carme, while S/2003 J6 has an orbit similar to J12 Ananke.
} 

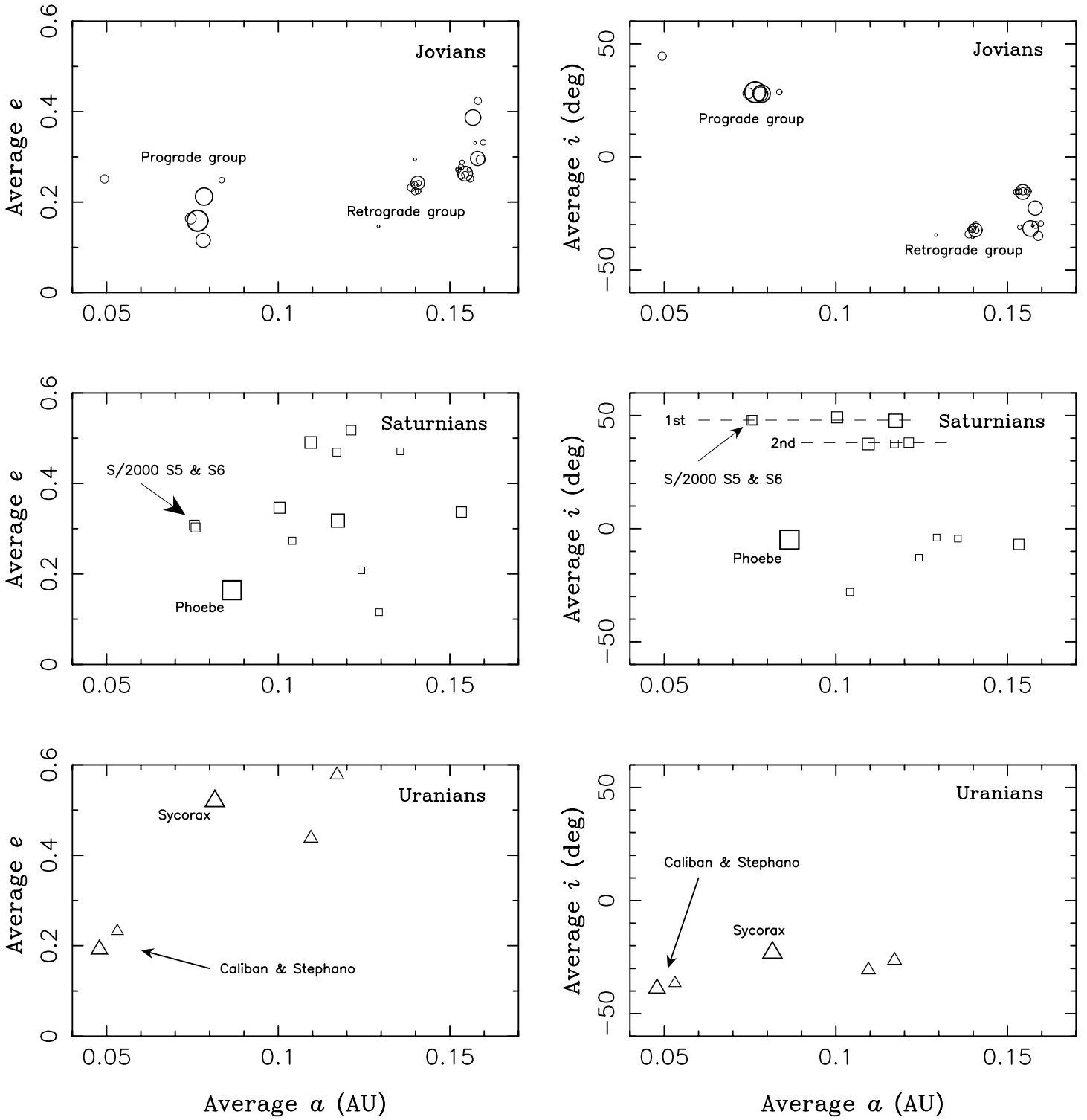

FIg. 13.-Average orbital elements of the irregular moons $(\langle a\rangle,\langle e\rangle,\langle i\rangle)$. For retrograde orbits, we show $\langle i\rangle-180^{\circ}$, where $\langle i\rangle$ is their average inclination with respect to the planetary invariable plane. Several groups of moons with similar average orbits are readily visible in this plot. The Jovian irregulars form two groups of prograde and retrograde moons. The retrograde group shows several subclusters (e.g., the Carme and Ananke families; see Fig. 14). The Saturnian irregulars form two groups of moons with nearly the same orbital inclinations (first and second inclination groups; Gladman et al. 2001). The irregular moons of Uranus S/1997 U1 Caliban and S/1999 U2 Stephano also have similar orbits. Some of these groups may have been formed by catastrophic disruptions of large parent moons. Gladman et al. (2001) proposed that the group of Saturnian moons with inclinations similar to that of Phoebe originated in a cratering impact on Phoebe.

the group of Ananke (the Ananke family). These velocities are much more compatible with a $\delta V$ expected from simple collisional breakups than the velocities computed for the whole retrograde group. We thus propose that the retrograde group of Jovian irregular moons witnessed a more complicated collisional history than thought before. It seems likely that at least two low-velocity fragmentations of two distinct precursor bodies occurred within this group. The spectral differences between Carme and Ananke suggest that these immediate precursor bodies correspond to two captured satellites instead of having a common ancestor (Luu 1991; Sykes et al. 2000; Brown 2000; Rettig et al. 2001).

Assuming that the prograde satellite group formed by a single collisional breakup, it is also hard to explain why its members have widely dispersed $\langle a\rangle$ and $\langle e\rangle$ but nearly the same value of $\langle i\rangle$ (here and in the following, angle brackets denote the average elements). According to Gauss's equations, this would suggest that either $\cos (f+\omega) \sim 0$ (collision occurred far from the nodes of the parent moon's orbit) or $\delta V_{W}$ was a factor of $\sim 5$ smaller than the other two components of $\delta V$. The latter alternative would require that the impactor and the parent moon were moving on nearly coplanar orbits at the time of their collision and that the fragments were anisotropically ejected from the site of the breakup with small $\delta V_{W}$ velocities.

Saturn.-Following Gladman et al. (2001), we classify the irregular moons of Saturn into groups of similar average orbital inclinations. We thus have the first satellite inclination group (S/2000 S4, S10, and S11), the second satellite 


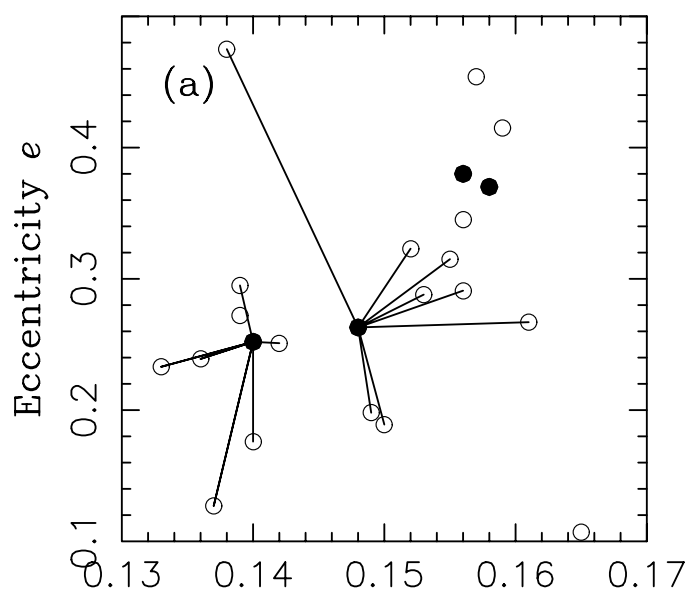

Semimajor axis a (AU)

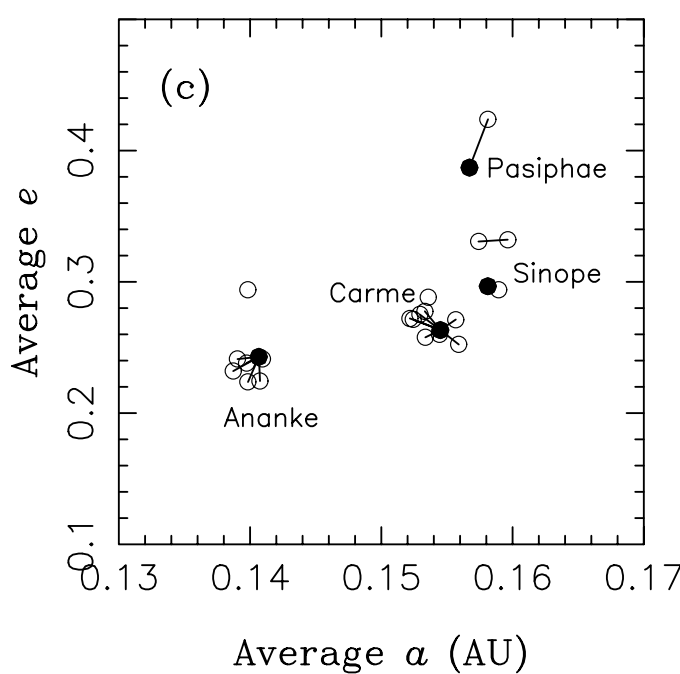

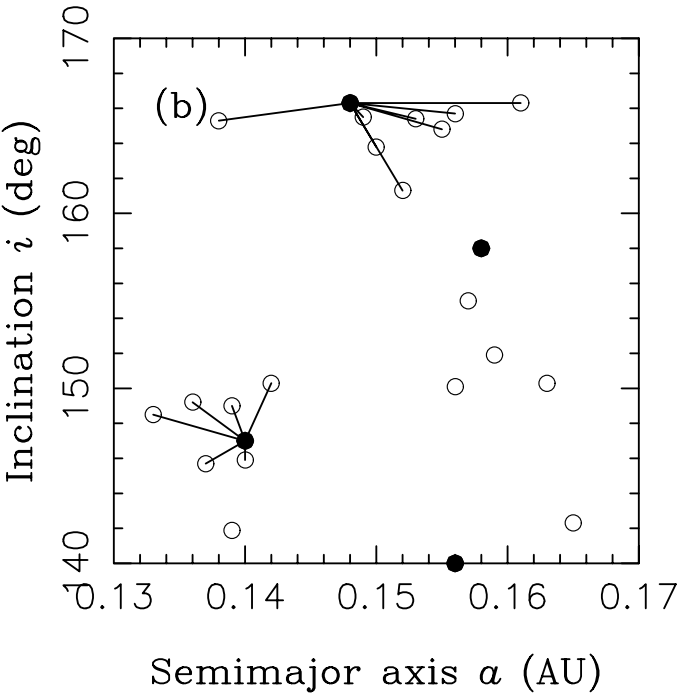

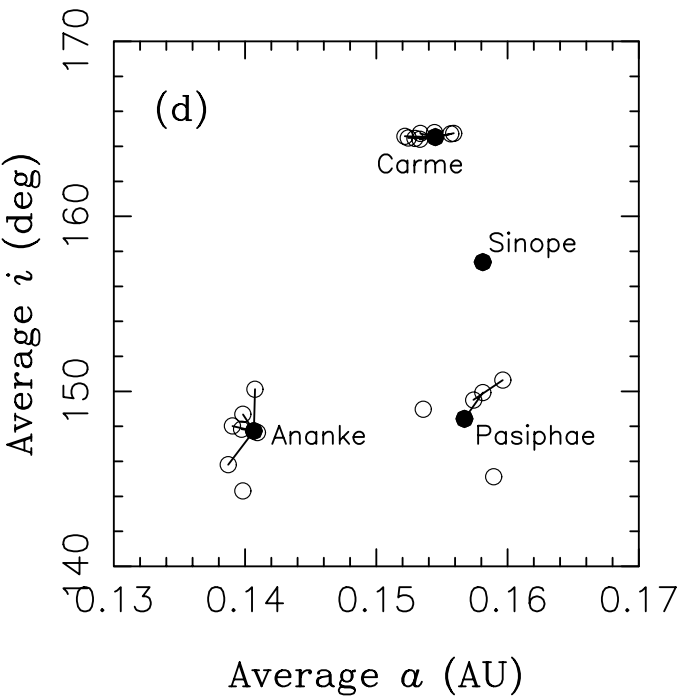

FIG. 14.-The retrograde group of Jovian irregular moons: $(a, b)$ instantaneous osculating orbits of the moons; $(c, d)$ average orbits. In $(a, b)$, the orbital

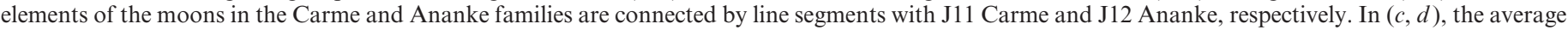

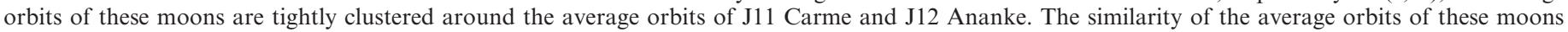
suggests their common origin.

inclination group (S/2000 S2, S3, S5, and S6), and a rather loosely clustered Phoebe group (Phoebe and S/2000 S1, S7, $\mathrm{S} 9$, and S12). Our first and second satellite groups correspond to the $34^{\circ}$ and $46^{\circ}$ inclination groups of Gladman et al. (2001). Here, however, because our reference plane is the invariable plane of the solar system instead of the ecliptic used by Gladman et al., the average inclinations $\langle i\rangle$ we compute for the first and second inclination groups are $\sim 38^{\circ}$ and $\sim 48^{\circ}$, respectively.

We believe that the moons in each of these inclination groups probably do not have a common collisional origin, unless (1) asymmetric and large-magnitude ejection velocity fields occurred and/or (2) collisions occurred early and some subsequent primordial mechanism modified the semimajor axes. Otherwise, it is hard to reconcile the magnitude and components of $\delta V$ computed from the Gauss equations with the current understanding of collisional breakups (see Michel et al. 2001, 2002). We obtain $30 \mathrm{~m} \mathrm{~s}^{-1} \lesssim \delta V \lesssim 60 \mathrm{~m} \mathrm{~s}^{-1}$ and $\delta V_{W} /$ $\left[\left(\delta V_{T}^{2}+\delta V_{R}^{2}\right) / 2\right]^{1 / 2} \sim 5$ for the first inclination group, $60 \mathrm{~m} \mathrm{~s}^{-1} \lesssim \delta V \lesssim 300 \mathrm{~m} \mathrm{~s}^{-1}$ and $\delta V_{W} /\left[\left(\delta V_{T}^{2}+\delta V_{R}^{2}\right) /\right.$ $2]^{1 / 2} \sim 10$ for the second inclination group, and $100 \mathrm{~m}$ $\mathrm{s}^{-1} \lesssim \delta V \lesssim 400 \mathrm{~m} \mathrm{~s}^{-1}$ for the Phoebe group. One characteristic of the irregular satellite systems we find from this calculation is that $\delta V_{W}$ derived from Gauss's equations is consistently smaller (by a factor of $\gtrsim 5$ ) than the other two components of $\delta V$. This seems to contradict a simple collisional scenario that would produce similar components of $\delta V$, as observed for young asteroid families in the main belt (Nesvorný et al. 2002a).

S/2000 S5 and S/2000 S6 have nearly identical average orbits (Fig. 13 and Table 4 ). The differences of average orbital elements between these two moons are $3.5 \times 10^{-4} \mathrm{AU}$, $5.5 \times 10^{-3}$, and 0.1 in $\langle a\rangle,\langle e\rangle$, and $\langle i\rangle$, respectively. We will show in $\S 9$ that the orbital evolution of both moons is characterized by the Kozai resonance (Kozai 1962). These are the first two moons ever discovered having this kind of the orbital behavior (see also Cuk et al. 2002). Other examples 
of the Kozai resonance in our solar system include Pluto and some near-Earth asteroids and ecliptic comets.

Uranus. - Because of the small number of Uranus's irregular moons known at this moment, is impossible to tell whether their orbits can be grouped in some way. We might be tempted to link S/1997 U1 Caliban and S/1999 U2 Stephano, but this association is purely speculative. In fact, the orbital distribution of Uranus's irregular moons is statistically consistent with a random distribution of the retrograde orbits within the stability limits determined by our surveys.

\section{COLLISIONS AMONG IRREGULAR MOONS}

A satellite family can be formed when a large object (parent body) collides with a high-speed impactor (projectile). If the energy of this collision is high enough, the parent body is broken into pieces and the pieces are dispersed in space. They assume orbits similar to the parent body's preimpact orbit. If the observed groups of satellites originated in this way, it is likely that their parent bodies were bound to the planet prior to the disruptive collision. Otherwise, it is hard to explain why the member moons of at least some satellite families have very similar orbits that are so strongly gravitationally bound to the planet. The collision of two stray bodies on unbound orbits may also produce satellites; such satellites, however, are not expected to have similar orbits.

In past eons, the dominant type of collisions in the irregular satellite systems of the Jovian planets were presumably the collisions among the moons. The current flux of external impactors such as the ecliptic and nearly isotropic comets (Zahnle et al. 2003), escaped Trojans or asteroids, etc., is far too low to produce many collisions. The situation was very probably different in early stages of the formation of the solar system, when the outer solar system may have been inhabited by a population of small bodies with a total mass of $\approx 50-200 M_{\text {Earth }}$ (Hahn \& Malhotra 1999). At that time, the rate of collisions with external impactors may have been important. Thus, the satellite families may date back to ancient collisions.

Here we concentrate on collisions among irregular satellites, which are also a plausible way to produce satellite families. Our method to compute the rate of collisions among moons is similar to that of Kessler (1981). He calculates that a population of bodies with the same $a, e$, and $i$ and random $\lambda, \varpi$, and $\Omega$ has the space density distribution

$$
P(r, \beta)=\frac{1}{2 \pi^{3} a^{2} r} \frac{1}{\sqrt{e^{2}-(r / a-1)^{2}} \sqrt{\cos ^{2} \beta-\cos ^{2} i}}
$$

with the limits

$$
\begin{gathered}
a(1-e) \leq r \leq a(1+e), \\
-i \leq \beta \leq i .
\end{gathered}
$$

Here $r=\left(x^{2}+y^{2}+z^{2}\right)^{1 / 2}$ and $\beta=\arcsin (z / r)$, where $x, y$, and $z$ are the Cartesian coordinates. We normalized the above distribution to a total number of one body in the population. Thus, $P(r, \beta) \Delta x \Delta y \Delta z$ is the probability that the body is located within a box of size $\Delta x \times \Delta y \times \Delta z$ centered at $(x, y, z)$.

Assuming random angles, we compute the probability of collision per unit time between a body with orbital elements $\left(a_{1}, e_{1}, i_{1}\right)$ and a body with orbital elements $\left(a_{2}, e_{2}, i_{2}\right)$ as

$$
\begin{aligned}
P_{\text {col }}= & \pi\left(R_{1}+R_{2}\right)^{2} 2 \pi \int_{r_{\min }}^{r_{\max }} \int_{\beta_{\min }}^{\beta_{\max }} P_{1}(r, \beta) P_{2}(r, \beta) \\
& \times V_{\text {col }}(r, \beta) r^{2} \cos \beta d r d \beta,
\end{aligned}
$$

where $P_{1}$ and $P_{2}$ are the probability distributions (eq. [10]) assuming $\left(a_{1}, e_{1}, i_{1}\right)$ and $\left(a_{2}, e_{2}, i_{2}\right)$, respectively; $V_{\mathrm{col}}=$ $\left|\boldsymbol{V}_{1}-\boldsymbol{V}_{2}\right|$, where $\boldsymbol{V}_{1}$ and $\boldsymbol{V}_{2}$ are the orbital velocities of the two bodies; $r_{\min }=\max \left[a_{1}\left(1-e_{1}\right), \quad a_{2}\left(1-e_{2}\right)\right], \quad r_{\max }=$ $\min \left[a_{1}\left(1+e_{1}\right), a_{2}\left(1+\mathrm{e}_{2}\right)\right], \beta_{\max }=\min \left(i_{1}, i_{2}, 180^{\circ}-i_{1}\right.$, $\left.180^{\circ}-i_{2}\right)$, and $\beta_{\min }=-\beta_{\max }$; and $\sigma=\pi\left(R_{1}+\mathrm{R}_{2}\right)^{2}$ is the total cross section of the two moons, with $R_{1}$ and $R_{2}$ being their radii. The orbital velocities $\boldsymbol{V}_{1}$ and $\boldsymbol{V}_{2}$ at $(x, y, z)$ are computed from $\left(a_{1}, e_{1}, i_{1}\right)$ and $\left(a_{2}, e_{2}, i_{2}\right)$, respectively.

We evaluate the above integral numerically (Press et al. 1992). We verified that using this algorithm, we calculate values of the collision probabilities that agree within $10 \%$ with the collision probabilities reported by Bottke \& Greenberg (1993) and Manley, Migliorini, \& Bailey (1998) for their test cases of asteroidal and cometary orbits. Next we used this algorithm to compute the rates of collisions among the irregular moons. For each moon, we assumed that its orbital elements $(a, e, i)$ were fixed and equal to the averaged values (Tables 3 and 4). Moreover, we neglected the gravitational focusing of the moons. Gravitational focusing is negligible in the regime of sizes and encounter velocities investigated here.

Jupiter.-Table 5 shows the collision rates and mean collision velocities that characterize collisions between each pair of irregular moons in the Jovian prograde group. In total, about five satellite-satellite collisions would occur in this group over $4.5 \mathrm{Gyr}$. This tells us that some of the moons we see today may be by-products of past satellite-satellite collisions. Moreover, Himalia has a rather large probability of colliding with other moons in the group (0.67-1.46 collisions [!] with each known prograde moon per $4.5 \mathrm{Gyr}$ ). Consequently, Himalia must have accreted a number of small irregular moons, former members of the prograde group. We thus propose that the population of small irregular satellites in prograde orbits may have been larger in the past and partially vanished by cratering impacts on the larger members of the prograde group.

Using scaling laws for the impact energy threshold needed to catastrophically disrupt a body $\left(Q_{D}^{*}\right.$; Benz \& Asphaug 1999), we calculate that Himalia would get disrupted by an impact by Elara ( 1.5 impacts per $4.5 \mathrm{Gyr})$. Similarly, Elara would be disrupted by an impact by Lysithea and/or by Leda. These latter events, however, are less probable. In broader sense, these results suggest that the prograde group of Jovian irregular moons could have had a rich collisional history in the past $4.5 \mathrm{Gyr}$ and that its current structure could have derived from past satellite-satellite collisions.

The retrograde group of Jovian irregular satellites has a much lower rate of collisions among themselves due to longer orbital periods of these moons and due to the large volume of space occupied by their orbits. In total, $\lesssim 1$ collision would have occurred in this group over 4.5 Gyr. Mutual collisions between moons in the retrograde group thus seem to be only marginally relevant. Note, however, that if the total number of Jovian retrograde moons is much larger than the 
TABLE 5

Collision Rates, Velocities, and Impact Energies for Pairs of Member Moons of the Prograde Jovian Group

\begin{tabular}{|c|c|c|c|c|c|c|c|}
\hline ID 1 & ID 2 & $\begin{array}{c}10^{14} P_{\mathrm{col}} / \sigma \\
\left(\mathrm{km}^{-2} \mathrm{yr}^{-1}\right)\end{array}$ & $\begin{array}{l}\text { Collisions } \\
\text { per } 4.5 \mathrm{Gyr}\end{array}$ & $\begin{array}{c}V_{\mathrm{col}} \\
\left(\mathrm{km} \mathrm{s}^{-1}\right)\end{array}$ & $\begin{array}{c}Q^{*} \\
\left(10^{8} \mathrm{ergs} \mathrm{g}^{-1}\right)\end{array}$ & $\begin{array}{c}Q_{D}^{*} \\
\left(10^{8} \mathrm{ergs} \mathrm{g}^{-1}\right)\end{array}$ & $Q^{*} / Q_{D}^{*}$ \\
\hline \multirow[t]{4}{*}{$2 \ldots \ldots \ldots \ldots \ldots$} & 3 & 3.9 & 0.95 & 1.56 & 0.2 & 9.2 & 0.02 \\
\hline & 4 & 4.1 & 0.38 & 1.65 & 1.1 & 4.3 & 0.26 \\
\hline & 5 & 3.9 & 0.11 & 1.49 & 8.7 & 1.5 & 5.8 \\
\hline & 6 & 3.4 & 0.01 & 1.76 & 2.9 & 0.5 & 5.8 \\
\hline \multirow[t]{3}{*}{$3 \ldots \ldots \ldots \ldots \ldots$} & 4 & 3.1 & 1.46 & 1.64 & 25.8 & 9.2 & 2.8 \\
\hline & 5 & 3.5 & 1.07 & 1.51 & 2.2 & 9.2 & 0.24 \\
\hline & 6 & 3.3 & 0.66 & 1.73 & 0.004 & 9.2 & 0.0004 \\
\hline \multirow[t]{2}{*}{ 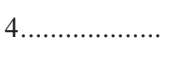 } & 5 & 2.6 & 0.36 & 1.62 & 13.3 & 4.4 & 3.0 \\
\hline & 6 & 2.6 & 0.18 & 1.68 & 0.02 & 4.4 & 0.005 \\
\hline $5 \ldots \ldots \ldots \ldots \ldots \ldots$ & 6 & 1.9 & 0.03 & 1.73 & 0.2 & 1.5 & 0.13 \\
\hline
\end{tabular}

Notes.-The columns are identification numbers of the moons (ID 1 and ID 2), "intrinsic" collision probability $\left(P_{\mathrm{col}} / \sigma, P_{\mathrm{col}}\right.$ from eq. [13]), number of collisions expected for each pair over $4.5 \mathrm{Gyr}$, mean collision velocity $\left(V_{\mathrm{col}}\right)$, mean specific impact energy $\left(Q^{*}\right)$, critical specific energy needed to catastrophically break up the larger moon in the pair and disperse the fragments $\left(Q_{D}^{*}\right.$, Benz \& Asphaug 1999), and the ratio of the specific to critical energies $\left(Q^{*} / Q_{D}^{*}\right)$. A catastrophic breakup of the target is expected for $Q^{*} / Q_{D}^{*}>1$.

25 currently known objects (Sheppard \& Jewitt 2002), collisions in the retrograde group may still be important.

We have noticed a few cases of possible collisions between prograde and retrograde Jovian irregular moons. For example, there is a $10 \%$ chance that Elara (prograde) is hit by S/2000 J8 (retrograde) over 4.5 Gyr. Despite the small size of S/2000 J8 $(D \sim 6 \mathrm{~km})$, the specific energy ( $Q^{*}$; see, e.g., Holsapple et al. 2003) of such a collision is nearly $10^{8}$ ergs $\mathrm{g}^{-1}$ due to the large impact speeds between prograde and retrograde objects $\left(\approx 6 \mathrm{~km} \mathrm{~s}^{-1}\right)$. This is about $25 \%$ of the $Q_{D}^{*}$ needed to disrupt (and disperse) Elara, according to the empirical scaling law we are using (Benz \& Asphaug 1999). We caution, however, that the scaling law derived by Benz \& Asphaug predicts larger $Q_{D}^{*}$ than values computed by other authors (Housen \& Holsapple 1990; Holsapple 1994; Melosh \& Ryan 1997). If smaller values of $Q_{D}^{*}$ better represent the real threshold for a breakup, a collision between Elara and S/2000 J8 $\left(Q^{*} \sim 10^{8} \mathrm{ergs}^{-1}\right)$ would have been catastrophic.

Our method predicts a zero collision probability for moons with average orbits that do not cross. For example, the average orbits of Pasiphae and Elara do not cross but barely miss each other by only $0.001 \mathrm{AU}$ (Table 2); equation (13) used with average orbital elements then gives $P_{\text {col }}=0$ for this couple. In reality, however, Pasiphae is not collisionally decoupled from Elara and other moons of the prograde group, because when the evolutions of the orbits are taken into account, the orbit of Pasiphae can reach down to planetocentric distances as small as $0.045 \mathrm{AU}$. To compute the collision probability of Pasiphae with the prograde moons, we made use of our $10^{8} \mathrm{yr}$ integration of orbits of the irregular moons. At each time step (each $1000 \mathrm{yr}$ ), we evaluated $P_{\text {col }}$ (eq. [13]) using the instantaneous orbital elements of Pasiphae and the prograde irregular moons. The average rate of collisions was then computed by averaging these collision probabilities over the $10^{8} \mathrm{yr}$ integration time span.

Using this method, we computed that Pasiphae and Elara have a $14 \%$ probability of colliding over the age of the solar system. Similarly, Pasiphae and Himalia have a $27 \%$ probability of colliding. If we account for all collisions of Pasiphae with the moons in the prograde group that would be disruptive for Pasiphae $\left(Q^{*}>Q_{D}^{*}\right.$, with $Q_{D}^{*}$ again from Benz \&
Asphaug 1999), we end up with a $\approx 50 \%$ chance that Pasiphae survives over the age of the solar system. Other moons in the retrograde group have smaller but nonnegligible rates of disruptions by impacts from the prograde group. This collisional coupling between the prograde and retrograde groups suggests that both populations may have evolved by collisions in the past.

As a by-product of the above computation, we have checked whether the use of average elements in equation (13) is justified for those moons with overlapping average orbits. For example, using the average orbits we previously computed that Elara and Himalia should collide 1.46 times over the age of the solar system with $\left\langle V_{\text {col }}\right\rangle \approx 1.64 \mathrm{~km} \mathrm{~s}^{-1}$. Using the instantaneous orbital elements of Elara and Himalia to compute $P_{\text {col }}$, and averaging $P_{\text {col }}$ over $10^{8} \mathrm{yr}$, we obtain 1.5 collisions per $4.5 \mathrm{Gyr}$ and a $1.65 \mathrm{~km} \mathrm{~s}^{-1}$ impact speed. Both these values are in good agreement with the result calculated by using the average orbits. The use of average elements is, however, not precise for average orbits that barely cross each other. For example, S/2000 J8 and Elara have about $5.8 \%$ probability of colliding over $4.5 \mathrm{Gyr}$ according to our refined calculation, while the method based on average orbits gives an $8.1 \%$ probability. Also the impact speeds differ somewhat: 6.25 versus $6.01 \mathrm{~km} \mathrm{~s}^{-1}$, respectively. Thus, the actual collision rates and collision velocities may be somewhat different from the values shown in Tables 5, 6, and 7 for those average orbits that have a small overlap. As a matter of fact, S/2000 J8 has larger collision rates with Himalia $(\approx 17 \%)$ than with Elara, even though the average orbits of S/2000 J8 and Himalia are noncrossing. The larger cross section of Himalia is the main factor here.

The rate of collisions among the irregular moons calculated here raises the question whether the satellite families (§ 6) formed in the early solar system by impacts from planetesimals in the residual protoplanetary disk or whether they can be of more recent origin. For example, the Ananke and Carme families probably date back to the primordial stage of the solar system, because the current collision rates among moons in the retrograde Jovian group are too low. Conversely, we believe that the prograde group of Jovian irregulars may have been produced more recently and 
TABLE 6

Collision Rates, Velocities, and Specific Impact Energies for Pairs of Saturn's Irregular Moons

\begin{tabular}{|c|c|c|c|c|c|c|c|}
\hline ID 1 & ID 2 & $\begin{array}{c}10^{14} P_{\mathrm{col}} / \sigma \\
\left(\mathrm{km}^{-2} \mathrm{yr}^{-1}\right)\end{array}$ & $\begin{array}{l}\text { Collisions } \\
\text { per } 4.5 \mathrm{Gyr}\end{array}$ & $\begin{array}{c}V_{\mathrm{col}} \\
\left(\mathrm{km} \mathrm{s}^{-1}\right)\end{array}$ & $\begin{array}{c}Q^{*} \\
\left(10^{8} \mathrm{ergs} \mathrm{g}^{-1}\right)\end{array}$ & $\begin{array}{c}Q_{D}^{*} \\
\left(10^{8} \mathrm{ergs} \mathrm{g}^{-1}\right)\end{array}$ & $Q^{*} / Q_{D}^{*}$ \\
\hline \multirow[t]{4}{*}{$1 \ldots \ldots \ldots \ldots \ldots$} & 3 & 1.6 & 1.17 & 2.97 & 0.16 & 21.2 & 0.007 \\
\hline & 4 & 0.51 & 0.01 & 1.48 & 34.3 & 0.98 & 35. \\
\hline & 6 & 0.36 & 0.02 & 1.39 & 5.2 & 2.2 & 2.4 \\
\hline & 8 & 0.43 & 0.01 & 1.63 & 24.0 & 1.3 & 18.5 \\
\hline \multirow[t]{3}{*}{$2 \ldots \ldots \ldots \ldots \ldots$} & 3 & 1.6 & 1.16 & 2.99 & 0.09 & 21.2 & 0.004 \\
\hline & 6 & 0.36 & 0.01 & 1.39 & 2.9 & 2.2 & 1.3 \\
\hline & 8 & 0.46 & 0.01 & 1.63 & 13.4 & 1.3 & 10.3 \\
\hline \multirow[t]{8}{*}{$3 \ldots \ldots \ldots \ldots$} & 4 & 0.81 & 0.64 & 3.14 & 0.56 & 21.2 & 0.03 \\
\hline & 5 & 0.49 & 0.33 & 0.94 & 0.002 & 21.2 & $<0.001$ \\
\hline & 6 & 0.66 & 0.61 & 3.16 & 3.3 & 21.2 & 0.15 \\
\hline & 7 & 0.67 & 0.47 & 3.33 & 0.04 & 21.2 & 0.002 \\
\hline & 8 & 0.59 & 0.49 & 3.28 & 1.0 & 21.2 & 0.05 \\
\hline & 9 & 0.49 & 0.36 & 3.33 & 0.16 & 21.2 & 0.008 \\
\hline & 10 & 0.29 & 0.20 & 0.47 & $<0.001$ & 21.2 & $<0.001$ \\
\hline & 12 & 1.5 & 1.04 & 0.68 & $<0.001$ & 21.2 & $<0.001$ \\
\hline $4 \ldots \ldots \ldots \ldots \ldots \ldots$ & 6 & 0.28 & 0.02 & 1.24 & 13.2 & 2.2 & 6.0 \\
\hline $5 \ldots \ldots \ldots \ldots \ldots \ldots$ & 6 & 0.54 & 0.02 & 2.72 & 2.1 & 2.2 & 0.95 \\
\hline \multirow[t]{3}{*}{$6 \ldots \ldots \ldots \ldots \ldots$} & 8 & 0.18 & 0.01 & 1.20 & 21.2 & 2.2 & 9.6 \\
\hline & 10 & 0.37 & 0.01 & 2.48 & 1.2 & 2.2 & 0.55 \\
\hline & 11 & 0.36 & 0.01 & 2.45 & 1.2 & 2.2 & 0.55 \\
\hline
\end{tabular}

evolved to its current structure by many other collisions. In this sense, the prograde Jovian group may be an analog of asteroid families in the main belt.

Saturn.-Saturn's system of irregular moons differs from that of Jupiter. The prograde and retrograde orbits of the Saturnian irregular moons show a large range of semimajor axes. Thus, collisions are possible between nearly any pair of moons (Table 6). Except for collisions involving Phoebe, however, the collision rates are usually low as a consequence of smaller orbital velocities than those of the Jovian irregulars, and due to the small sizes of the satellites. In fact, the collision rate of any pair of moons in the Saturnian satellite system (Phoebe excluded) is smaller than 0.02 collisions per pair of bodies per $4.5 \mathrm{Gyr}$.

Impacts of small moons on Phoebe are thus the main manifestation of the current collisional activity in the Saturnian irregular satellite system. In total, we calculate that some six to seven impacts of irregular moons on Phoebe occur over 4.5 Gyr using the currently known population. Most of these impacts are impacts by prograde satellites $(\approx 75 \%$; Table 6$)$. Despite the large impact velocities of the prograde moons on retrograde Phoebe $\left(\approx 3 \mathrm{~km} \mathrm{~s}^{-1}\right)$, none of these impacts would disrupt Phoebe, because typically $Q^{*} \ll Q_{D}^{*}$. The largest impact energy would characterize the impact of the $45 \mathrm{~km}$ diameter moon S/2000 S3; 0.6 such impacts occur over $4.5 \mathrm{Gyr}$ with $Q^{*} \sim 0.15 Q_{D}^{*}$.

Phoebe's surface should thus bear marks of the intense bombardment by impactors from prograde satellite orbits. We predict that the craters produced by these impacts will be a few tens of kilometers in diameter and should be clearly visible during the Cassini flyby of Phoebe in 2004. Moreover, intense cratering should have occurred on Phoebe's surface by impacts by smaller irregular moons. For comparison, Zahnle et al. (2003) found that impacts of ecliptic comets on satellites strongly dominate over impacts by nearly isotropic comets. Zahnle et al. estimated that one 20 $\mathrm{km}$ crater should be created on Phoebe by an ecliptic comet impact every 11-27 billion years. This cratering rate is small compared with the cratering rate on Phoebe from the satellite impacts. We thus predict that most large craters that the Cassini spacecraft images may reveal on Phoebe were either created during primordial epochs or resulted from the prograde satellites' impacts at later times. Moreover, ejecta from Phoebe's surface produced by impacts may be the

TABLE 7

Collision Rates, Velocities, and Specific Impact Energies for Pairs of Uranus's Irregular Moons

\begin{tabular}{|c|c|c|c|c|c|c|c|}
\hline ID 1 & ID 2 & $\begin{array}{c}10^{14} P_{\mathrm{col}} / \sigma \\
\left(\mathrm{km}^{-2} \mathrm{yr}^{-1}\right)\end{array}$ & $\begin{array}{l}\text { Collisions } \\
\text { per } 4.5 \mathrm{Gyr}\end{array}$ & $\begin{array}{c}V_{\mathrm{col}} \\
\left(\mathrm{km} \mathrm{s}^{-1}\right)\end{array}$ & $\begin{array}{c}Q^{*} \\
\left(10^{8} \mathrm{ergs} \mathrm{g}^{-1}\right)\end{array}$ & $\begin{array}{c}Q_{D}^{*} \\
\left(10^{8} \operatorname{ergs~g}^{-1}\right)\end{array}$ & $Q^{*} / Q_{D}^{*}$ \\
\hline \multirow[t]{3}{*}{$1 \ldots \ldots \ldots \ldots \ldots \ldots$} & 2 & 2.1 & 0.15 & 0.57 & 0.61 & 3.2 & 0.19 \\
\hline & 3 & 0.87 & 0.32 & 0.72 & 3.3 & 8.3 & 0.40 \\
\hline & 5 & 0.23 & 0.02 & 0.68 & 2.9 & 3.2 & 0.91 \\
\hline \multirow{3}{*}{$2 \ldots \ldots \ldots \ldots$} & 3 & 0.56 & 0.12 & 0.67 & 0.10 & 8.3 & 0.12 \\
\hline & 4 & 0.21 & 0.006 & 0.55 & 4.5 & 1.3 & 3.5 \\
\hline & 5 & 0.24 & 0.007 & 0.65 & 6.3 & 1.3 & 4.8 \\
\hline \multirow[t]{2}{*}{$3 \ldots \ldots \ldots \ldots \ldots \ldots \ldots$} & 4 & 0.11 & 0.03 & 0.46 & 0.16 & 8.3 & 0.02 \\
\hline & 5 & 0.13 & 0.03 & 0.49 & 0.18 & 8.3 & 0.02 \\
\hline $4 \ldots \ldots \ldots \ldots \ldots \ldots$ & 5 & 0.07 & 0.003 & 0.42 & 8.9 & 1.3 & 6.8 \\
\hline
\end{tabular}


source of the dark material that is responsible for the large albedo variations on Iapetus (Cruikshank et al. 1983; Bell, Cruikshank, \& Gaffey 1985; Jarvis et al. 2000). Indeed, dust material escaping Phoebe's gravity evolves due to PoyntingRobertson drag to later impact Iapetus from a retrograde orbit (Burns et al. 1996; Hamilton 1997).

Uranus.-The irregular moons of Uranus have low but nonnegligible collision rates: 0.69 collisions are expected to occur among its five irregular moons over 4.5 Gyr. Such collisions are characterized by small impact velocities (typically $0.58 \mathrm{~km} \mathrm{~s}^{-1}$ ). This small rate of collisions among Uranus's irregulars may explain why, unlike Saturn's and Jupiter's irregular moons, the irregular moons of Uranus do not show clear groupings of objects in the space of the average orbital elements (Fig. 13) - an expected outcome of catastrophic collisions among the moons. On the other hand, it is also possible that we see only the largest members of groupings that consist of yet-to-be-discovered sub-10 km moons and that have primordial origins. In any case, we find that very few collisions would occur among the Uranian irregular moons over 4.5 Gyr. We conclude that both the observations and the theoretical modeling suggest that the collisional activity in the Uranus satellite system was probably unimportant for shaping the size and orbital distribution of the currently observed moons.

One of the general characteristics that can be noted in the orbital distribution of known irregular moons is that the closer that the planet is to the Sun, the more distant are its irregular satellites when expressed in units of the Hill radius of the planet. Can this be a consequence of the collisional evolution of the moons? More distant planets have larger Hill spheres than Jupiter. Moons with $a=f R_{j}^{\mathrm{H}}$, where $0<f<1$, also have lower orbital speeds for larger $j$ and fixed $f$. Thus, the rate of collisions of satellites at a fractional distance $f$ from the $j$ th planet is lower for larger $j$. Does this allow the moons of distant planets to be collisionally long-lived at small $f$ ?

We performed a simple experiment. We placed the Uranian system of irregular moons at Jupiter. In doing so, we left the eccentricities and inclinations of the moons' orbits fixed and also kept the values of the semimajor axes fixed in terms of the Hill radius (i.e., $f=$ const). We then computed the rates of collisions among the new orbits. This experiment showed that Uranus's irregular moons, when placed at Jupiter, are collisionally short-lived. The collisional rates were a factor of $\approx 14$ larger than the collisional rates of the real Uranian system; about eight to nine catastrophic collisions occurred among the five moons of the displaced Uranian system over 4.5 Gyr. These collisions would eliminate most of the bodies and leave the region depleted. Similarly, the Saturnian system of irregular moons, when placed at Jupiter, experienced $\approx 25$ catastrophic collisions over $4.5 \mathrm{Gyr}$.

We thus propose that the observed semimajor axis distribution of the irregular satellites may have resulted from mutual collisions among moons at small $a$. In any case, the Jovian irregular moons with $a \lesssim(0.1-0.2) R_{5}^{\mathrm{H}}$ and in crossing orbits must be collisionally short-lived unless they are very few and/or very small. Only one known irregular moon actually has $a$ within this range (S/1975 J1). Gladman et al. (2001) proposed another explanation for the semimajor-axis distribution of the irregular moons. They suggested that such a distribution developed in early stages of the solar system formation when moons became captured and evolved by gas drag in the circumplanetary gas nebulae (Pollack et al. 1979).

\section{PROGRADE-RETROGRADE COLLISIONS AS AN EVOLUTIONARY PROCESS}

We have shown in the previous section that collisions between prograde and retrograde irregular moons in the Jovian satellite system may occur. Such collisions are, however, unlikely even on gigayear timescales when the collision rates are computed using the current populations: we estimated that the largest retrograde moon, Pasiphae, has about a $50 \%$ chance of colliding with a moon in the prograde group over $4.5 \mathrm{Gyr}$; other retrograde moons have smaller collision rates. For this reason, the prograde and retrograde groups of moons are now nearly collisionally decoupled. Does this also mean that they were collisionally decoupled in the past? Not necessarily. In fact, the present orbital structures may be in part a product of past collisions between prograde and retrograde moons.

Figure 15 shows the rate of collisions between small test satellites orbiting a planet at selected distances and the largest irregular moon of the planet (Himalia, Phoebe, Sycorax, and Nereid for Jupiter, Saturn, Uranus, and Neptune, respectively). We used $e=0.25$ and $i=160^{\circ}$ for the test satellite orbits at Jupiter, $e=0.3$ and $i=40^{\circ}$ at Saturn, $e=0.3$ and $i=30^{\circ}$ at Uranus, and $e=0.3$ and $i=160^{\circ}$ at Neptune. The orbital inclinations of these test orbits were chosen so that the sense of orbital motion of the moons is opposite to that of the large irregular moons. Moreover, we used $e$ and $i$ that are typical for the systems of observed satellites at each planet except for Uranus and Neptune, where no moons are seen to have the desired inclinations (except $\mathrm{S} / 2002 \mathrm{~N} 1$ ). Note that the collision probability is not a very sensitive function of $e$ and $i$; the results we obtained are thus representative for a large range of $e$ and $i$.

The question we want to answer is whether a small irregular satellite moving in a test orbit would last over the age of the solar system without having collided with any other larger moon. The collision of a small (say, a few kilometers) object with a body of the size of Himalia, Phoebe, Sycorax, or Nereid would obviously be destructive for the small moon. Moreover, since such impacts occur at hypervelocity speeds (e.g., $\gtrsim 6 \mathrm{~km} \mathrm{~s}^{-1}$ for the Jupiter retrograde moons impacting prograde Himalia, $\gtrsim 3 \mathrm{~km} \mathrm{~s}^{-1}$ for the Saturn prograde moons impacting retrograde Phoebe; Table 6), they would leave large craters on the surfaces of the large moons.

Most Jovian retrograde irregular moons with 0.05 $\mathrm{AU} \lesssim a \lesssim 0.11$ AU would impact Himalia over $4.5 \mathrm{Gyr}$. (This semimajor axis range corresponds to $\gtrsim 50 \%$ impact probability in Fig. 15a.) Thus, if such a hypothetical population of irregular moons existed in the past, it would be largely depleted at present by past collisions with the large moons in the prograde group. The currently known moons in the retrograde group are located outside the outer edge of the determined depletion zone, at $0.13-0.16 \mathrm{AU}$. They are thus mostly protected from collisions with Himalia and other large moons of the prograde group. Note that only collisions with Himalia were shown in Figure 15; if collisions with other large moons of the prograde group were considered, the outer limit of the depletion zone would move to $a \sim 0.12 \mathrm{AU}$. We conclude that the dichotomy of the Jovian irregular satellite system (i.e., groups of prograde and retrograde moons widely separated in a) may have 

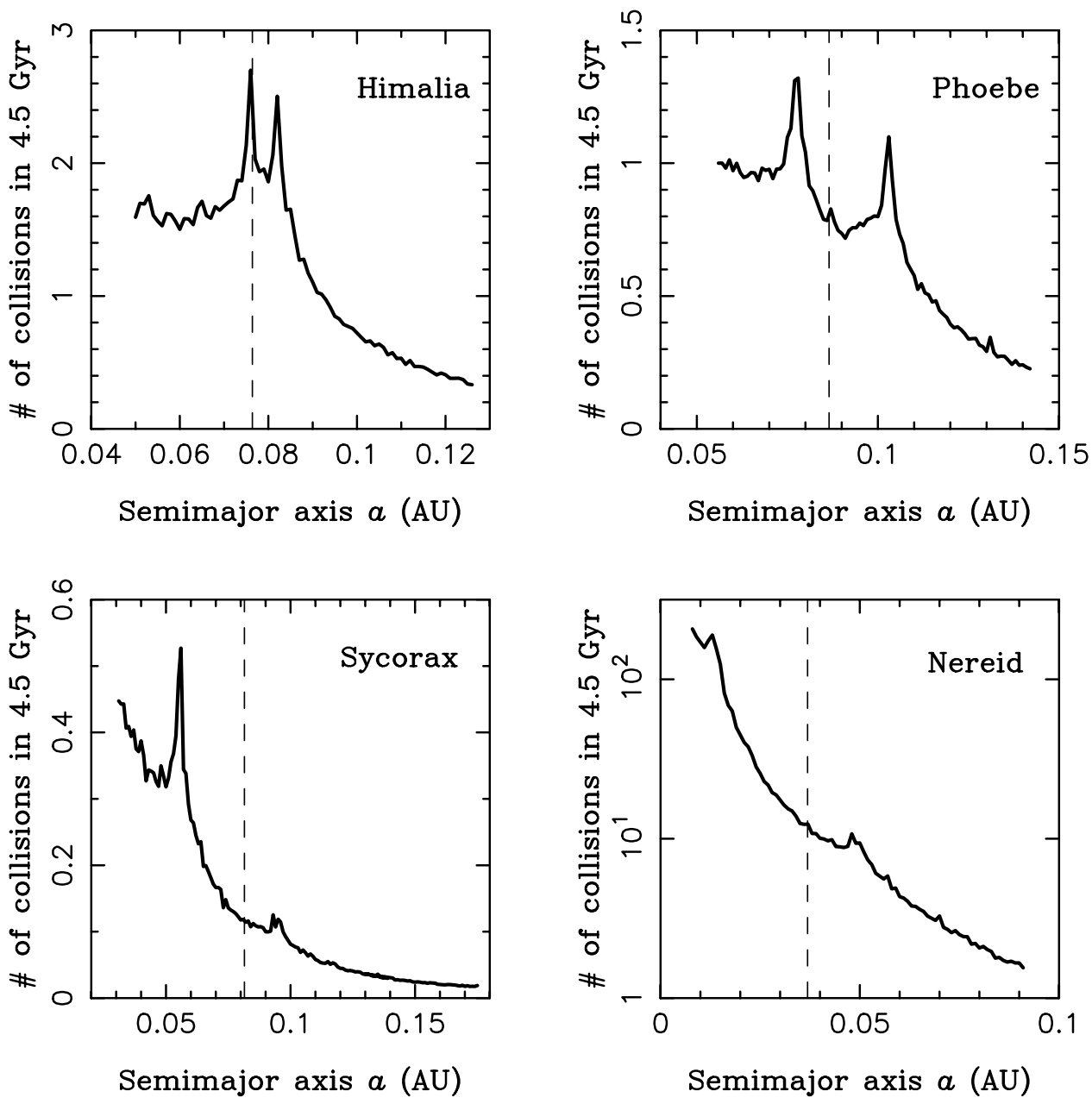

FIG. 15.-Number of collisions per 4.5 Gyr suffered by small test irregular moons of Jupiter, Saturn, Uranus, and Neptune with Himalia, Phoebe, Sycorax, and Nereid, respectively (semimajor-axis locations of these moons are denoted by dashed lines), as a function of their semimajor axis. The small test moons were assumed to orbit their parent planets in the opposite direction to that of the large irregular moons. The hypothetical primordial populations of such small irregular moons must have been depleted at those semimajor axes at which the number of collisions is substantial. Note the high collision rates of the Neptunian moons with Nereid.

developed through collisions between the prograde and retrograde moons over the last $4.5 \mathrm{Gyr}$, with prograde Himalia playing a major role in this process.

The Saturnian irregular satellite system is different: the prograde and retrograde moons in this system show no semimajor axis groupings. Most of them move on crossing orbits. Collisions are thus possible between nearly any prograde-retrograde pair of moons. We calculate that the collision rates with Phoebe are lower than the rate of collisions with Himalia (compare Fig. 15a with 15b). This may explain the difference of the currently observed orbital distributions of the Jovian and Saturnian irregular moons. We believe that the Saturnian prograde satellite system must have been more numerous in the past and should have been depleted by a factor of $30 \%-100 \%$ at $0.05 \mathrm{AU} \lesssim a \lesssim 0.15 \mathrm{AU}$ by collisions with Phoebe. The moons removed from this region must have produced craters on Phoebe's surface.

The rate of collisions between test prograde satellites and Sycorax in the Uranian system is far too low to explain the lack of prograde irregular moons at this planet (Fig. 15c). Only at $a \sim 0.05$ AU would nearly $50 \%$ of small prograde moons have been collisionally destroyed. The result is much more interesting for Neptune, where Nereid, due to its large cross section, has a large number of collisions with retrograde moons at $0.01-0.1$ AU (Fig. 15d). Thus, it may be argued that the lack of such satellites ${ }^{14}$ resulted from collisions with Nereid over the past $4.5 \mathrm{Gyr}$.

To understand this effect better, we studied a larger range of test orbits.

Figure 16 shows the number of collisions between a small test satellite and Nereid as a function of the small satellite's orbital elements. There is a large number of collisions at small $a$. The moons with $i \sim i_{\text {Nereid }} \approx 10^{\circ}$ and $i \sim$ $180^{\circ}-i_{\text {Nereid }}$ have larger collision rates with Nereid than the moons with $30^{\circ} \lesssim i \lesssim 150^{\circ}$. Moreover, the retrograde moons have a larger rate of collisions with Nereid than the prograde moons. This distribution of collision rates is expected from simple geometric considerations and equation (13).

We thus propose that Nereid could have eliminated a large fraction of the primordial population of small irregular moons at Neptune. The effect of collisions with Nereid is, however, limited to small distances from Neptune

\footnotetext{
${ }^{14}$ Indeed, the newly discovered irregular moons of Neptune have $a \sim 0.13-0.15 \mathrm{AU}$.
} 

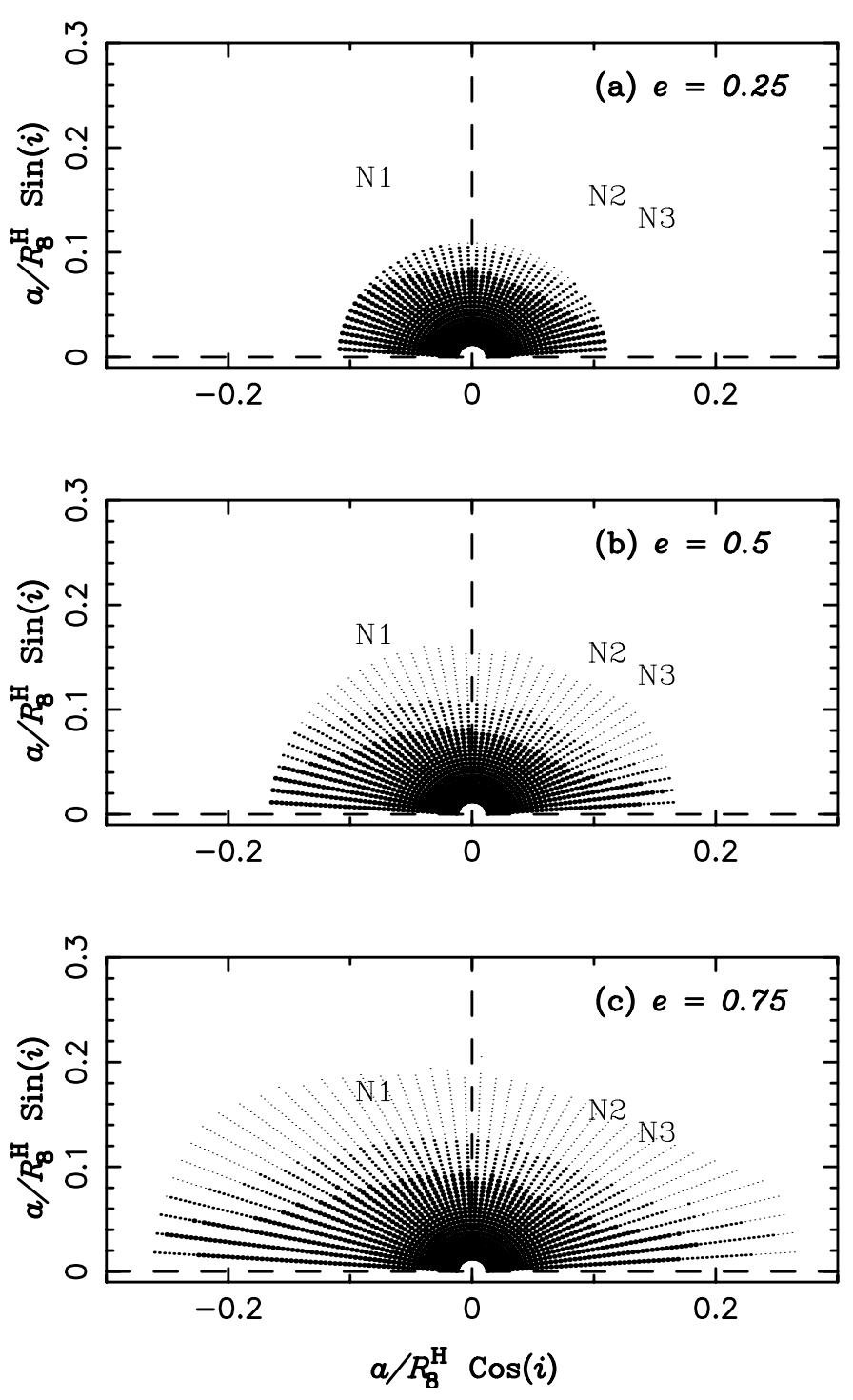

FIG. 16.-Collisions of hypothetical irregular satellites of Neptune with Nereid. Three symbol sizes were used. From the smallest to the largest size, the symbols denote the orbits with larger than 18\%, 39\%, and $63 \%$ probabilities of colliding with Nereid in 4.5 Gyr. Small Neptunian moons at small semimajor axes impact Nereid on timescales that are short compared with the age of the solar system. Orbits of three new irregular satellites of Neptune (S/2002 N1, N2, and N3; Holman et al. 2003) are located at larger distances from Neptune, and are safe from collisions with Nereid unless they have very high eccentricities. Orbital eccentricities of these new moons are yet to be determined.

$\left[a \lesssim(0.1-0.15) R_{8}^{\mathrm{H}}\right]$. At larger distances, moons may be collisionally decoupled from Nereid and may be collisionally (and dynamically) long-lived. This is the case for S/2002 $\mathrm{N} 1, \mathrm{~N} 2$, and N3, which all have $a \sim 0.18 R_{8}^{\mathrm{H}}$. These newly discovered moons present an interesting constraint on the scenario proposed by Goldreich et al. (1989), in which Triton is captured and sweeps through the Neptune's Hill sphere, because the survival of S/2002 N1, N2, and N3 may be difficult in this scenario.

\section{RESONANCES}

Orbital resonances of bodies in our solar system are common. They are often related to processes acting at early epochs. For example, it has been proposed that Plutinos were captured into their resonant orbits by the radial migration of Neptune during late stages of the formation of the solar system (Malhotra 1995). Other resonances may be more recent: for example, the Galilean satellites might have evolved to their current configuration (characterized by the Laplace resonance) by tidal effects. ${ }^{15}$ In this section, we study the orbits of the irregular satellites to determine whether some of them move in resonant orbits.

We used our $10^{8} \mathrm{yr}$ orbital histories of the 50 irregular moons to compute the time evolution of resonant angles that are allowed by the d'Alembert rules (e.g., Morbidelli 2002). This evolution was then visually inspected to see whether some resonant angle oscillates around a fixed value. To select resonant angles that slowly evolve with time, we first determined the frequencies $g$ and $s$ for each moon (the mean apsidal and nodal frequencies) and combined the corresponding angles ( $\varpi$ and $\Omega$ ) so that the resulting angle changes slowly. We then completed this selection by combinations of the planetary angles so that the constructed resonant angle obeys the d'Alembert rules and checked whether it shows resonant behavior.

As a result of this systematic search, we found that several irregular satellites actually have resonant orbits (Table 8). Some of these cases were previously known: (1) Pasiphae and Sinope show periods of resonant behavior, where $\phi=\varpi-2 \Omega+\varpi_{5}$ librates about $180^{\circ}$ (Whipple \& Shelus 1993; Saha \& Tremaine 1993). (2) Sinope (and possibly also $\mathrm{S} / 2001 \mathrm{~J} 11)$ interact with the $6: 1$ mean motion resonance, where $n-6 n_{5} \sim 0$ (Saha \& Tremaine 1993). (3) The orbits of S/2001 J10, S/2000 S5, and S/2000 S6 are locked in the Kozai resonance, where $\omega$ librates about $90^{\circ}$ with a full amplitude of about $60^{\circ}$ (see also Carruba et al. 2002a). (4) The apsis of $\mathrm{S} / 2000 \mathrm{~S} 3$ becomes temporarily locked to that of Saturn (Vashkov'yak 2001; Ćuk et al. 2002); this resonance is known as $\nu_{6}$ in the asteroidal dynamics literature (Morbidelli 2002). We discuss some of these results below.

Figure 17 shows the evolution of the resonant arguments $\phi=\varpi-\Omega+\varpi_{5}$ for Pasiphae and Sinope. Initially, Pasiphae's orbit is locked in the secular resonance and $\phi$ oscillates with $\approx 180^{\circ}$ amplitude about $180^{\circ}$. In later times, the amplitude of $\phi$ shows stochastic variations. At $t \sim 2.7 \times 10^{7} \mathrm{yr}$, when $\phi$ starts circulating, the orbit escapes from the resonance. Sinope's orbit has intermittent periods of resonant and nonresonant behavior.

Saha \& Tremaine (1993) proposed that the resonant orbits of Pasiphae and Sinope resulted from some kind of slow dissipative orbital evolution (such as that due to gas drag). Otherwise, it is difficult to explain why orbits of two of the four largest Jovian retrograde moons are located in tiny resonant volumes of the orbital space. Figure 17 shows that Pasiphae's and Sinope's orbits evolve from the resonant to near-resonant locations and vice versa on $10^{7} \mathrm{yr}$ timescales. For this reason, and if Pasiphae and Sinope really became captured in the secular resonance by the usual resonant capture mechanism (Henrard 1983), the dissipative evolution of orbits over the resonant locations must have occurred in less then $\sim 10^{7} \mathrm{yr}$ and the dissipation must have stopped shortly after capture occurred. Otherwise, according to Figure 17, the captured orbits would evolve

\footnotetext{
${ }^{15}$ Note, however, that their primordial migration due to interaction with a proto-satellite disk may have produced a similar result (Peale \& Lee 2002; Canup \& Ward 2002).
} 
TABLE 8

Irregular Satellites Found to OCCUPy or Interact with a Resonance

\begin{tabular}{|c|c|c|c|}
\hline ID & Satellite & Type of Resonance & References \\
\hline $16 \ldots \ldots \ldots \ldots \ldots$ & J8 (Pasiphae) & $\begin{array}{l}g-2 s+g_{5}=0 \\
g-2 s+g_{5}=0\end{array}$ & $\begin{array}{l}\text { Saha \& Tremaine 1993; Whipple \& Shelus } 1993 \\
\text { Saha \& Tremaine 1993; Whipple \& Shelus } 1993\end{array}$ \\
\hline $17 \ldots \ldots \ldots \ldots \ldots$ & J9 (Sinope) & $n-6 n_{5}=0$ & Saha \& Tremaine 1993 \\
\hline $21 \ldots \ldots \ldots \ldots . . .$. & $\mathrm{S} / 2001 \mathrm{~J} 10$ & $g-s=0$ (Kozai) & \\
\hline $29 \ldots \ldots \ldots \ldots . . .$. & $\mathrm{S} / 2001 \mathrm{~J} 11$ & $n-6 n_{5}=0(?)$ & \\
\hline $4 \ldots \ldots \ldots \ldots \ldots . .$. & $\mathrm{S} / 2000 \mathrm{~S} 2$ & $g-g_{6}=0(?)$ & Ćuk et al. 2002 \\
\hline $6 \ldots \ldots \ldots \ldots \ldots$ & $\mathrm{S} / 2000 \mathrm{~S} 3$ & $g-g_{6}=0$ & Ćuk et al. 2002 \\
\hline $1 \ldots \ldots \ldots \ldots \ldots$ & $\mathrm{S} / 2000 \mathrm{~S} 5$ & $g-s=0$ (Kozai) & Carruba et al. 2002a \\
\hline $2 \ldots \ldots \ldots \ldots \ldots$ & $\mathrm{S} / 2000 \mathrm{~S} 6$ & $g-s=0$ (Kozai $)$ & Carruba et al. 2002a \\
\hline
\end{tabular}

NotE.-The third column denotes the type of the resonance. The fourth column gives a reference for cases that have been known previously or were found recently by independent studies.

from their resonant locations and would continue their orbital decay toward the planet. This sets an upper limit on the timescale of dissipative evolution that produced the captures. On the other hand, the dissipative evolution must have been slow enough so that capture can actually occur. This assumes that $T_{\text {cross }} \gg T_{\phi}$, where $T_{\text {cross }}$ is the time to
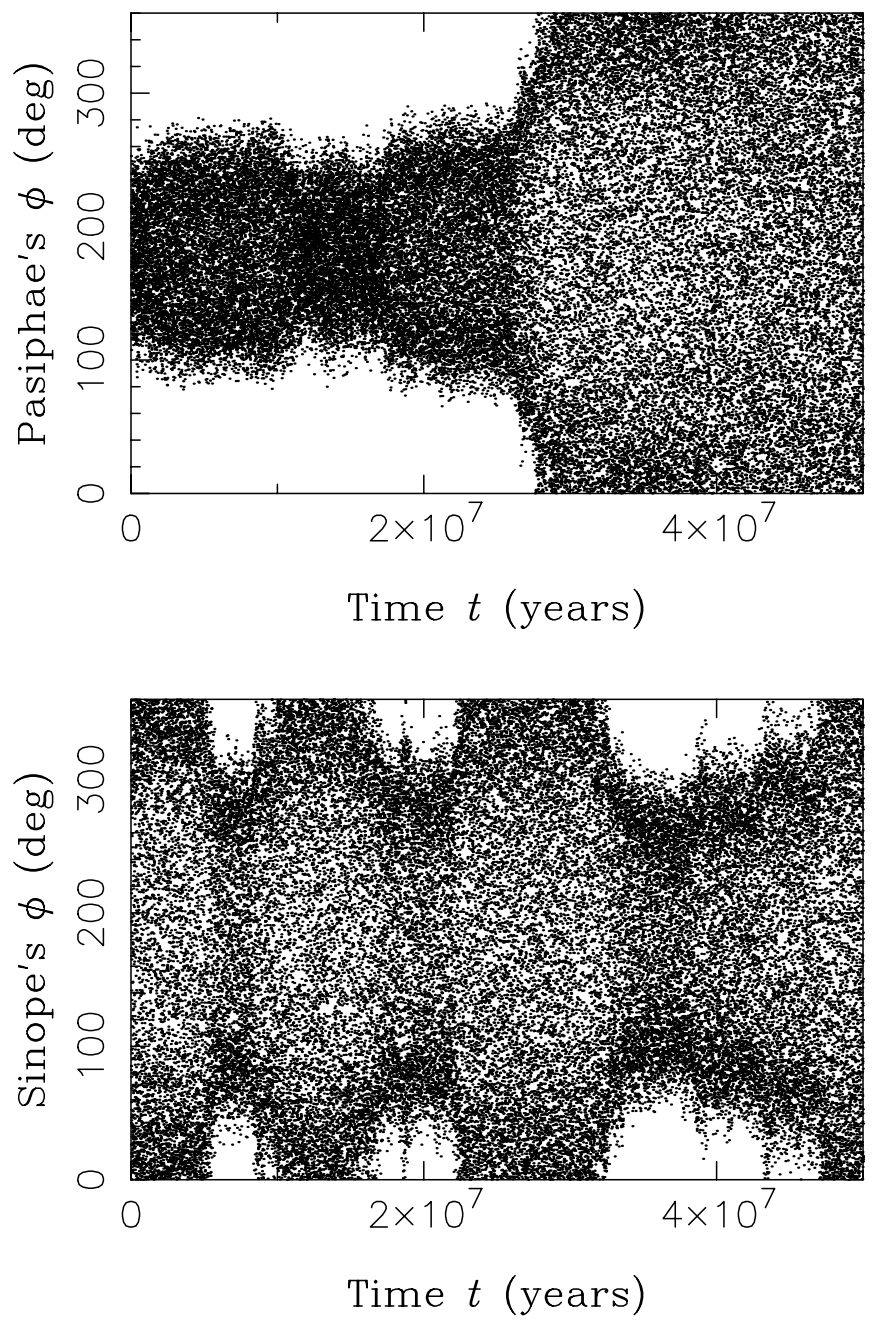

FIG. 17.-Evolution of the resonant arguments $\phi=\varpi-\Omega+\varpi_{5}$ for two irregular moons of Jupiter: (a) Pasiphae and (b) Sinope. The orbits of both satellites show periods of resonant behavior, where $\phi$ librates around $180^{\circ}$. cross the resonance by the dissipative evolution and $T_{\phi}$ is the libration period of $\phi$. The quantity $T_{\phi}$ for Pasiphae is typically a few times 10,000 yr. Taken together, $10^{4}$ yr $\ll T_{\text {cross }} \lesssim 10^{7}$ yr. This sets a weak but still important constraint on the formation of the irregular satellites.

We did not find any other orbit among the 26 simulated retrograde irregular moons of Jupiter that showed resonant behavior similar to that of Pasiphae and Sinope, nor did we detect an orbit among any of the Jovian irregulars that were locked in one of the other tiny resonances. Although this conclusion is preliminary, because some of the orbits we used may have substantial errors, we believe that resonances are not as common among the retrograde Jovian moons as it might have seemed before. Similarly, resonances are also rare among the irregular moons of the other planets. On the other hand, among the large irregular moons of the gas-giant planets (Jupiter and Saturn), the orbits of Pasiphae, Sinope, and S/2000 $\mathrm{S} 3$ are resonant or near-resonant. Does this mean that $30-50 \mathrm{~km}$ diameter bodies, such as the three resonant irregular moons, suffered dissipative evolution with $T_{\text {cross }} \gg 10^{4} \mathrm{yr}$ and became captured, while smaller bodies evolved faster and "jumped" over the resonances? This size dependence of the capture efficiency would be expected, for example, in the case of gas drag.

We have found that three irregular satellites (from the total of 50) have orbits locked in the Kozai resonance. In two cases, S/2000 S5 and S/2000 S6, the nominal orbits that were used for our integration were good enough to be sure that this result is robust. In the case of S/2001 J10, which was discovered only recently (Sheppard et al. 2002), we are not so sure and will have to wait until the orbit improves with additional observations.

In all three cases, $\omega$ oscillates around $90^{\circ}$ with $60^{\circ}$ full amplitude (Fig. 18) that is nearly constant over $10^{8} \mathrm{yr}$. Only the orbit of S/2000 S5 suffers transitions that occasionally change the amplitude by $\approx 5^{\circ}$. These transitions, however, seem to follow a regular pattern and we do not expect them to accumulate to the degree that $\mathrm{S} / 2000 \mathrm{~S} 5$ could leave the Kozai resonance on $10^{9} \mathrm{yr}$ timescales. The resonant behavior of these orbits is thus very likely primordial. The fact that only a small fraction (three out of 50) of the irregular satellites had primordial orbits in the Kozai resonance probably means that the capture of the irregular moons was driven by mechanisms other than the Kozai resonance; otherwise, we would expect a larger fraction of the irregular moons to have this orbital behavior today. 

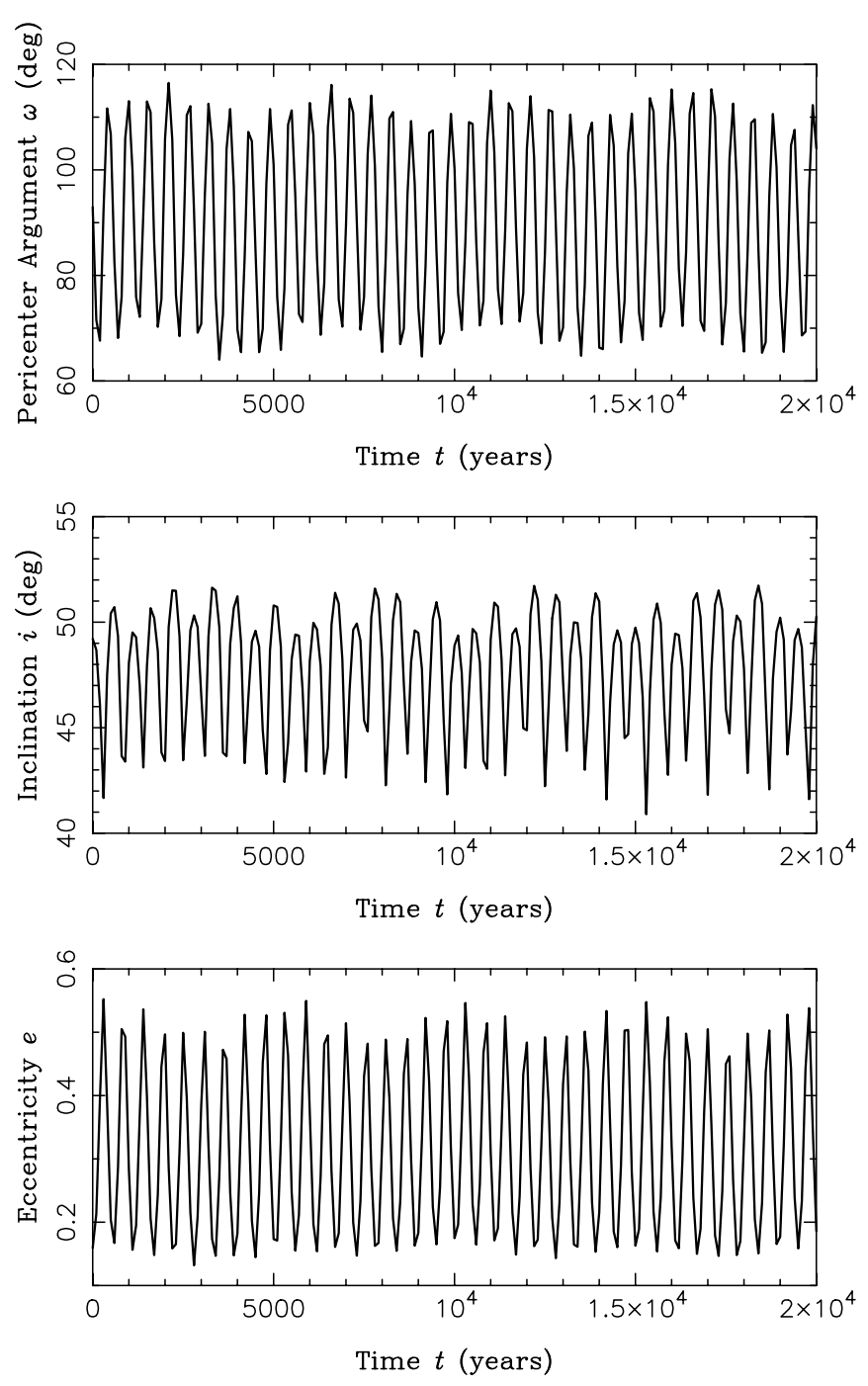

FIG. 18. - Resonant orbit of S/2000 S5. This orbit shows librations of $\omega$ around $90^{\circ}$, which is characteristic for the Kozai resonance.

We have found that Saturn's irregular satellite S/2000 S3 is affected by the secular resonance $g-g_{6}=0$, where $g$ and 9,6 are the moon's and planetary apsidal frequencies (see also Cuk et al. 2002). Periods of resonant behavior (Fig. 19), when the apsis of this moon's orbit oscillates with a large amplitude about that of Saturn, alternate with other epochs when the angle $\nu_{6}=\varpi-\varpi_{6}$ rotates over the full $360^{\circ}$ interval. The period of resonant oscillations of $\nu_{6}$ is typically $30,000-40,000 \mathrm{yr}$. The resonance occurs because the orbit of $\mathrm{S} / 2000 \mathrm{~S} 3$ has very slow, $g$ due to its high inclination $\left(48^{\circ}\right.$; Table 4). According to Cuk et al., S/2000 S2 also may be locked in the resonance $g-g_{6}=0$. We did not obtain the same result: our nominal orbit of S/2000 S2 is nonresonant. We acknowledge, however, that the orbit of S/2000 S2 needs to be improved before the final conclusion is drawn. Recently, Yokoyama et al. (2002) have developed a semianalytic model based on a fifth-order expansion in $a / a_{j}$ of the disturbing function that describes this type of resonance.

It is interesting to note that $\mathrm{S} / 2000 \mathrm{~S} 3$ has a $\approx 45 \mathrm{~km}$ diameter and is the largest among Saturn's irregulars except for Phoebe (Table 2). This once again points toward dissipative evolution of orbits with a size-dependent rate. In this
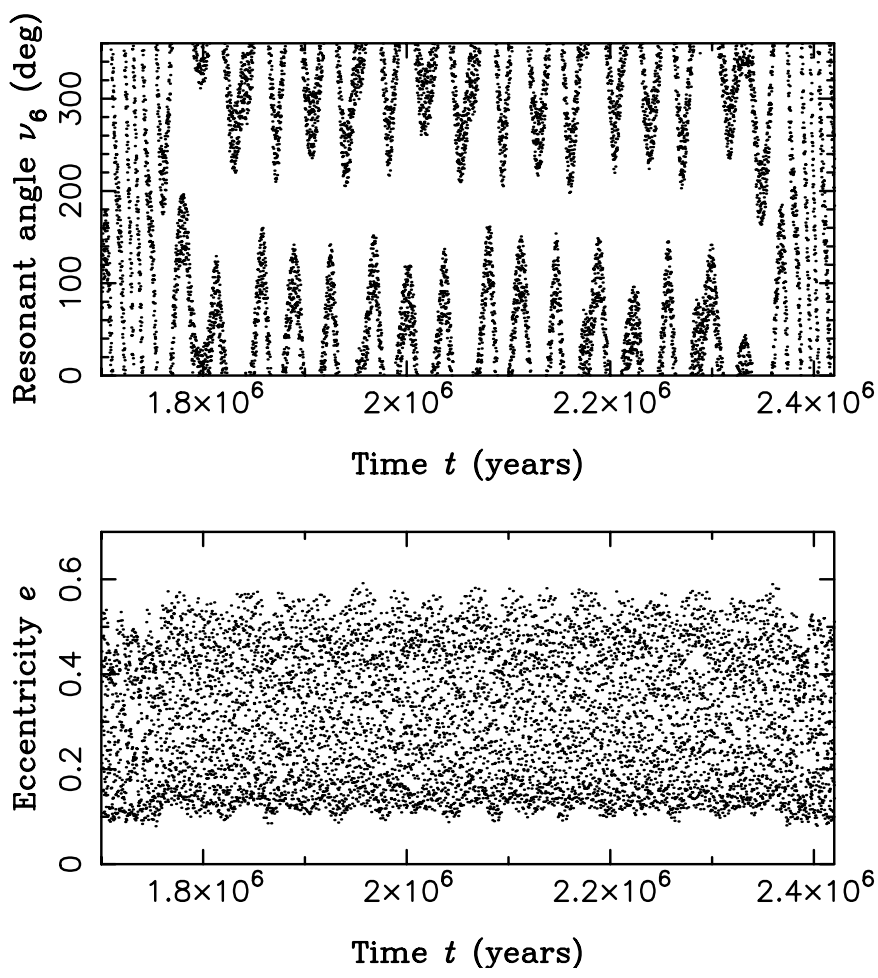

FIG. 19.- Resonant orbit of S/2000 S3. The apsis of this moon's orbit oscillates with a large amplitude about that of Saturn. The figure shows the time interval during which oscillations of the angle $\nu_{6}=\varpi-\varpi_{6}$ are particularly stable. At other times, $\nu_{6}$ shows intermittent behavior with resonant and nonresonant evolutions.

scenario, larger bodies such as $\mathrm{S} / 2000 \mathrm{~S} 3$ could have been captured into the resonances because their orbits evolved slowly enough to be captured, while smaller bodies with faster orbital decays jumped over resonances. On the other hand, the orbital decay of S/2000 S3 could not have lasted much longer than the typical time interval on which the intermittent behavior of $\nu_{6}$ occurs. Because $\nu_{6}$ typically switches from resonant to nonresonant mode in 30,000 500,000 time intervals (the period of resonant behavior shown in Fig. 19 is among the longest observed), the resonant crossing time must have been of this order or shorter.

Finally, concerning the long-term stability of the irregular satellites' orbits, all 50 integrated orbits were long-lived. In most cases, the orbits did not show any macroscopic chaotic evolutions in $10^{8} \mathrm{yr}$. In a few cases, however, the integrated orbit was clearly chaotic and underwent small transitions (e.g., the case of S/2000 S5 discussed above). We do not expect that these transitions could accumulate over gigayear timescales to a degree such that the satellite's orbit would become unbound.

\section{CONCLUSIONS}

Studies of the orbital and collisional evolution of the irregular moons of the Jovian planets may tell us about how this interesting component of the solar system inventory looked soon after the planets and their satellite systems formed. This, in turn, may help to solve the problem of the formation of the Jovian planets in our solar system and to better understand the formation of planets in general. 
In this paper, we obtained results that improved our understanding of the irregular moons in several respects:

1. We have shown that collisions among the irregular satellites might have been important for shaping their orbital and size-frequency distributions. For example, observations suggest that the closer that the planet is to the Sun, the more distant are its irregular satellites when expressed in Hill radii. We proposed that this distribution may have been produced by the collisional removal of inner irregular moons. We have shown that the irregular satellites of Saturn and Uranus would be collisionally short-lived if placed at Jupiter. We also argued that other characteristics of the irregular satellites, such the existence of two groups of prograde and retrograde moons at largely distinct semimajor axes at Jupiter, may have resulted from the collisional evolution. Collisions between prograde and retrograde irregular moons with similar semimajor axes are frequent and occur at large collision velocities. Thus, a hypothetical population of moons at the semimajor axes between the prograde and retrograde groups of Jupiter would have been substantially eroded by collisions with large moons of either group. We estimated that the typical rates of collisions among the irregular moons are such that $0.5-10$ disruptive collisions occur within a specific population over 4.5 Gyr. We believe that such a moderate current rate of collisions might have gradually developed by the collisional elimination of much larger primordial populations.

2. Phoebe should have suffered at least six or seven impacts from the population of the Saturnian irregular moons over the last 4.5 Gyr. Such impacts must have created craters on Phoebe's surface several tens of kilometers in size. By contrast, the current cratering rate by comets on Phoebe is at least 1 order in magnitude lower (Zahnle et al. 2003). We predict that most large craters on Phoebe, which will be imaged at sub-kilometer resolution by the Cassini spacecraft in 2004, were created by impacts of irregular moons. The number of craters on Phoebe's surface may thus tell us the total population of irregular satellites that formed at Saturn. Moreover, we suggested that the dark material excavated by satellite impacts from surface of Phoebe could have been transferred to Iapetus, where it covered the leading hemisphere of this moon and created the observed surface layer of low reflectance albedo (Jarvis et al. 2000).

3. We studied groups of satellites that show similar orbits. We argued that some of these groups are not compatible with simple scenarios of a single collisional breakup of one parent body per group. Otherwise, unusual ejection fields must be invoked to explain the current distribution of orbits. We discovered two new satellite families. The Carme family (nine members, possibly also S/2003 J1, J5, J9, J10, and J11) and Ananke family (seven members, possibly also S/2003 J6) were previously unresolved in the widely dispersed retrograde group of Jovian irregular moons. The ejection velocity fields computed for these families from the Gauss equations are compatible with simple collisional breakup scenarios. Moreover, S/1997 U1 Caliban and S/1999 U2 Stephano at Uranus have similar average orbits. These moons may have a common ancestor, but further observations are needed to see whether other fragments of the hypothetical parent moon exist at the same location.
4. We found that $\mathrm{S} / 2000 \mathrm{~S} 5, \mathrm{~S} / 2000 \mathrm{~S} 6$, and possibly also S/2001 J10 have orbits characterized by the Kozai resonance. Does this constrain the formation mechanism of these moons? Indeed, the locking of the argument of pericenter $\omega$ at $90^{\circ}$ might have been important to avoid certain orbital configurations that would produce very small pericenter distances during the capture. Note that S/2000 S5 and S/2000 S6 also have the smallest semimajor axes of all the known Saturnian irregular moons. In general, however, the Kozai resonance was not a driving mechanism for the capture, because otherwise a larger fraction of the irregular moons would be expected to reside within the resonance today. We verified that Pasiphae and Sinope show periods of resonant behavior, where $\phi=\varpi-2 \Omega+\varpi_{5}$ librates about $180^{\circ}$ (Whipple \& Shelus 1993; Saha \& Tremaine 1993). Similarly, the apsis of S/2000 S3 becomes temporarily locked to that of Saturn (Cuk et al. 2002). These resonant configurations suggest that the orbits of at least some irregular moons evolved by a dissipative mechanism in the past. We discussed the possible timescales on which this dissipation occurred.

5. We have shown that prograde-retrograde collisions between large irregular moons may have been an important mechanism that eliminated large fractions of the population of small irregular satellites at specific distances from the planets. Probably the most interesting case is that of Nereid. Small irregular moons of Neptune with $a \lesssim 0.15 R_{8}^{\mathrm{H}}$ are doomed to impact Nereid if they have large enough eccentricities to move on Nereid-crossing orbits. Thus, the lack of irregular satellites of Neptune with small $a$ may be attributed to Nereid's accretional cross section sweeping through space. Conversely, at $a \gtrsim 0.15 R_{8}^{\mathrm{H}}$, Neptune's moons are long-lived. The orbits of three newly discovered Neptune's irregular satellites (S/2002 N1, N2, and N3) satisfy this condition.

6. By means of numerical integrations and a semianalytic model, we studied the orbital dynamics and stability of the irregular moons of the Jovian planets. We have shown that satellites with $i \sim 90^{\circ}$ are unstable due to the effect of the Kozai resonance (see also Carruba et al. 2002a, 2002b). Moreover, we have shown that the distant retrograde orbits are stable to larger distances from planets than the prograde orbits. This asymmetry can be explained by the effect of the resonance between the apsis of the satellite's orbit and the planet's orbital revolutions around the Sun. This resonance, called the evection resonance in the theory of the Moon's orbit (e.g., Kaula \& Yoder 1976), excites eccentricities of the prograde moons with $a \gtrsim 0.5 R^{\mathrm{H}}$ to values close to 1 , thus driving them out of the Hill sphere. We have developed a semianalytic model of the distant satellites' orbits, which explains the orbital behavior of the irregular moons and validates our numerical results. The orbits of all known irregular moons are dynamically long-lived.

We thank Brett Gladman, Matt Holman, and Phil Nicholson for their suggestions to this work. This paper is based upon work supported by the National Science Foundation under grant 0074163 . We also thank the anonymous referee for constructive criticism of the manuscript. 


\section{REFERENCES}

Alvarellos, J. L., Zahnle, K. J., Dobrovolskis, A. R., \& Hamill, P. 2002, Icarus, 160, 108

Alvarellos, J. L. A. 1996, Master's thesis, San José State Univ.

Bell, J. F., Cruikshank, D. P., \& Gaffey, M. J. 1985, Icarus, 61, 192

Benz, W., \& Asphaug, E. 1999, Icarus, 142, 5

Bottke, W. F., \& Greenberg, R. 1993, Geophys. Res. Lett., 20, 879

Brown, M. E. 2000, AJ, 119, 977

Brown, R. H., Cruikshank, D. P., Pendleton, Y., \& Veeder, G. J. 1999, Icarus, 139, 374

Burns, J. A. 1986, in Satellites, ed. J. A. Burns \& M. S. Matthews (Tucson: Univ. Arizona Press), 1

Burns, J. A., Hamilton, D. P., Mignard, F., \& Soter, S. 1996, in IAU Colloq. 150 , Physics, Chemistry, and Dynamics of Interplanetary Dust, ed. B. A. S. Gustafson \& M. S. Hanner (ASP Conf. Ser. 104) (San Francisco: ASP), 179

Canup, R. M., \& Ward, W. R. 2002, AJ, 124, 3404

Carruba, V., Burns, J. A., Nicholson, P. D., Cuk, M., \& Jacobson, R. A. 2002a, BAAS, 34, Div. Planet. Sci. abstr. No. 35.01

Carruba, V., Burns, J. A., Nicholson, P. D., \& Gladman, B. J. 2002b, Icarus, 158, 434 (erratum 162, 230 [2003])

Colombo, G., \& Franklin, F. A. 1971, Icarus, 15, 186

Cruikshank, D. P., Bell, J. F., Gaffey, M. J., Brown, R. H., Howell, R., Beerman, C., \& Rognstad, M. 1983, Icarus, 53, 90

Ćuk, M., Burns, J. A., Carruba, V., Nicholson, P. D., \& Jacobson, R. A. 2002, BAAS, 34, Div. Dyn. Astron. abstr. No. 14.02

Gladman, B., et al. 2001, Nature, 412, 163

Gladman, B., Kavelaars, J. J., Holman, M., Petit, J.-M., Scholl, H., Nicholson, P., \& Burns, J. A. 2000, Icarus, 147, 320

Gladman, B., Nicholson, P., Burns, J., \& Kavelaars, J. J. 1999, IAU Circ. 7248

Gladman, B. J., Nicholson, P. D., Burns, J. A., Kavelaars, J. J., Marsden, B. G., Williams, G. V., \& Offutt, W. B. 1998, Nature, 392, 897

Goldreich, P. 1965, MNRAS, 130, 159

Goldreich, P., Murray, N., Longaretti, P. Y., \& Banfield, D. 1989, Science, 245,500

Hahn, J. M. \& Malhotra, R. 1999, AJ, 117, 3041

Hamilton, D. P. 1997, BAAS, 29, 1010

Hamilton, D. P., \& Krivov, A. V. 1997, Icarus, 128, 241

Hartmann, W. K. 1987, Icarus, 71, 57

Henrard, J. 1983, Icarus, 53, 55

Heppenheimer, T. A., \& Porco, C. 1977, Icarus, 30, 385

Holman, M. J., Kavelaars, J. J., Gladman, B. J., Petit, J.-M., Milisavljevic, D., \& Nicholson, P. D. 2001, BAAS, 33, Div. Planet. Sci. abstr. No. 37.11

Holman, M., Kavelaars, J., Grav, T., Fraser, W., \& Milisavljevic, D. 2003, IAU Circ. 8047

Holman, M., Kavelaars, J., \& Milisavljevic, D. 2002, IAU Circ. 7980

Holsapple, K., Giblin, I., Housen, K., Nakamura, A., \& Ryan, E. 2003, in Asteroids III, ed. W. F. Bottke, A. Cellino, P. Paolicchi, \& R. P. Binzel (Tucson: Univ. Arizona Press), 443

Holsapple, K. A. 1994, Planet. Space Sci., 42, 1067

Housen, K. R., \& Holsapple, K. A. 1990, Icarus, 84, 226

Jacobson, R. A. 2000, AJ, 120, 2679

2001, BAAS, 33, Div. Planet. Sci. abstr. No. 11.01

Jarvis, K. S., Vilas, F., Larson, S. M., \& Gaffey, M. J. 2000, Icarus, 146, 125

Kary, D. M., \& Dones, L. 1996, Icarus, 121, 207

Kaula, W. M., \& Yoder, C. F. 1976, in Lunar Science VII (Houston: Lunar Sci. Inst.), 440

Kessler, D. J. 1981, Icarus, 48, 39

Kinoshita, H., \& Nakai, H. 1999, Celest. Mech. Dyn. Astron., 75, 125

Kovalevsky, J. \& Sagnier, J.-L. 1977, in IAU Colloq. 28, Planetary

Satellites, ed. J. A. Burns (Tucson: Univ. Arizona Press), 43

Kozai, Y. 1962, AJ, 67, 579

Levison, H. F., \& Duncan, M. J. 1994, Icarus, 108, 18

Luu, J. 1991, AJ, 102, 1213

Malhotra, R. 1995, AJ, 110, 420

Manley, S. P., Migliorini, F., \& Bailey, M. E. 1998, A\&AS, 133, 437

Melosh, H. J., \& Ryan, E. V. 1997, Icarus, 129, 562
Michel, P., Benz, W., Tanga, P., \& Richardson, D. C. 2001, Science, 294, 1696

Michel, P., Tanga, P., Benz, W., \& Richardson, D. C. 2002, Icarus, 160, 10 (erratum 161, 198 [2003])

Morbidelli, A. 2002, Modern Celestial Mechanics (London: Taylor \& Francis)

Mosqueira, I., \& Estrada, P. R. 2001, BAAS, 33, Div. Planet. Sci. abstr. No. 25.03

2002a, Icarus, 163,198

2002b, Icarus, 163, 232

Murray, C. D., \& Dermott, S. F. 1999, Solar System Dynamics, Cambridge Univ. Press, Cambridge

Nesvorný, D., Bottke, W. F., Jr., Dones, L., \& Levison H. F. 2002a, Nature, 417,720

Nesvorný, D., Thomas, F., Ferraz-Mello, S., \& Morbidelli, A. 2002b, Celest. Mech. Dyn. Astron., 82, 323

Owen, T. C., Cruikshank, D. P., Dalle Ore, C. M., Geballe, T. R., Roush, T. L., \& de Bergh, C. 1999, Icarus, 139, 379

Peale, S. J., \& Lee, M. H. 2002, Science, 298, 593

Pisani, E., Dell'Oro, A., \& Paolicchi, P. 1999, Icarus, 142, 78

Pollack, J. B., Burns, J. A., \& Tauber, M. E. 1979, Icarus, 37, 587

Pollack, J. B., Lunine, J. I., \& Tittemore, W. C. 1991, in Uranus, ed. J. T. Bergstrahl, E. D. Miner \& M. S. Matthews (Tucson: Univ. Arizona Press), 469

Porco, C. C., et al. 2003, Science, 299, 1541

Press, W. H., Teukolsky, S. A., Vetterling, W. T., \& Flannery, B. P. 1992, Numerical Recipes in FORTRAN (2d ed.; Cambridge: Cambridge Univ. Press)

Rettig, T. W. Walsh, K \& \& Consolmagno, G. 2001, Icarus, 154, 313

Roy, A. E., \& Ovenden, M. W. 1954, MNRAS, 114, 232 1955, MNRAS, 115, 296

Saha, P., \& Tremaine, S. 1993, Icarus, 106, 549

Sheppard, S. S., \& Jewitt, D. C. 2002, BAAS, 34, Div. Planet. Sci. abstr. No. 23.04

Sheppard, S. S., Jewitt, D. C., Fernández, Y. R., Magnier, G., Marsden, B. G., Dahm, S., \& Evans, A. 2001, IAU Circ. 7555

Sheppard, S. S., Jewitt, D. C., Kleyna, J., Fernandez, Y. R., Hsieh, H. H., \& Marsden, B. G. 2003a, IAU Circ. 8087

Sheppard, S. S., Jewitt, D. C., Kleyna, J., Marsden, B. G., \& Jacobson, R. 2002, IAU Circ. 7900

Sheppard, S. S., \& Marsden, B. G. 2003b, IAU Circ. 8088

.2003c, IAU Circ. 8089

Šidlichovský, M. 1991, Bull. Astron. Inst. Czechoslovakia, 42, 116

Simonelli, D. P., Kay, J., Adinolfi, D., Veverka, J., Thomas, P. C., \& Helfenstein, P. 1999, Icarus, 138, 249

Stevenson, D. J., Harris, A. W., \& Lunine, J. I. 1986, in Satellites, ed. J. A

Burns \& M. S. Matthews (Tucson: Univ. Arizona Press), 39

Sykes, M. V., Nelson, B., Cutri, R. M., Kirkpatrick, J. D., Hurt, R., \& Skrutskie, M. F. 2000, Icarus, 143, 371

Tholen, D. J., \& Zellner, B. 1984, Icarus, 58, 246

Thomas, F., \& Morbidelli, A. 1996, Celest. Mech. Dyn. Astron., 64, 209

Thomas, P., Veverka, J., \& Helfenstein, P. 1991, J. Geophys. Res., 96, 19253

Thomas, P. C., Veverka, J., \& Helfenstein, P. 1996, in Neptune and Triton, ed. D. P. Cruikshank (Tucson: Univ. Arizona Press), 685

Touma, J., \& Wisdom, J. 1998, AJ, 115, 1653

Vashkov'yak, M. A. 2001, AZh Pisma, 27, 533 (English transl. Astron. Lett., 27, 455)

Whipple, A. L. \& Shelus, P. J. 1993, Icarus, 101, 265

Wisdom, J., \& Holman, M. 1991, AJ, 102, 1528

Wisdom, J., Holman, M., \& Touma, J. 1996, in Integration Algorithms and Classical Mechanics, ed. J. E. Marsden, W. G. Patrick, \& W. F. Shadwick (Fields Inst. Commun. 10) (Providence: Am. Math. Soc.), 217

Yokoyama, T., Santos, M. T., Cardin, G., Winter, O. C., \& Winter,

S. M. G. 2002, BAAS, 34, Div. Planet. Sci. abstr. No. 35.07

Yoshida, H. 1990, Phys. Lett. A, 150, 262

Zahnle, K., Schenk, P., Levison, H., \& Dones, L. 2003, Icarus, in press 